\title{
A Measurement of the Mass of the Top Quark in Lepton + Jets Events at $\mathrm{CDF}$
}

\author{
by
}

Erik Matthews Brubaker

B.A. (Lawrence University, Wisconsin) 1999

\begin{abstract}
A dissertation submitted in partial satisfaction of the requirements for the degree of Doctor of Philosophy

in

Physics

in the

GRADUATE DIVISION

of the

UNIVERSITY OF CALIFORNIA, BERKELEY
\end{abstract}

\author{
Committee in charge: \\ Professor Young-Kee Kim, Co-Chair \\ Professor James Siegrist, Co-Chair \\ Professor Stuart Freedman \\ Professor Joseph Cerny
}

Fall 2004 
The dissertation of Erik Matthews Brubaker is approved:

\begin{tabular}{lr}
\hline Co-Chair & Date \\
\hline Co-Chair & Date \\
\hline & Date \\
\hline & \\
\hline
\end{tabular}

University of California, Berkeley

Fall 2004 


\title{
A Measurement of the Mass of the Top Quark in Lepton + Jets Events at $\mathrm{CDF}$
}

\author{
Copyright 2004 \\ by
}

Erik Matthews Brubaker 


\begin{abstract}
by

Erik Matthews Brubaker

Doctor of Philosophy in Physics

University of California, Berkeley

Professor Young-Kee Kim, Co-Chair

Professor James Siegrist, Co-Chair
\end{abstract}

A Measurement of the Mass of the Top Quark in Lepton + Jets Events at CDF

This document presents a measurement of the top quark mass using the CDF run II detector at Fermilab. Colliding beams of protons and anti-protons at Fermilab's Tevatron $(\sqrt{s}=1.96 \mathrm{TeV})$ produce top/anti-top pairs, which decay to $W^{+} W^{-} b \bar{b}$; events are selected where one $W$ decays hadronically, and one $W$ decays to either $e$ or $\mu$ plus a neutrino. The data sample was collected between March 2002 and September 2003, and corresponds to an integrated luminosity of approximately $162 \mathrm{pb}^{-1}$. Thirty-seven candidate $t \bar{t}$ events are found with at least one $b$ jet identified by its displaced vertex. In each event, the best fit top quark invariant mass is determined by minimizing a $\chi^{2}$ for the overconstrained kinematic system. A likelihood fit of the reconstructed masses in the data sample to distributions from simulated signal and background events gives a top mass of $174.9_{-7.7}^{+7.1}$ (stat.) \pm 6.5 (syst.) $\mathrm{GeV} / c^{2}$. The dominant systematic error is due to uncertainties in the jet energy measurements.

Professor Young-Kee Kim

Dissertation Committee Co-Chair

Professor James Siegrist

Dissertation Committee Co-Chair 
To my inestimable wife

María Luz,

and to my son

Tomás Valentín,

born September 4, 2004. 


\section{Contents}

List of Figures $\quad$ iv

List of Tables $\quad$ vi

1 Introduction 1

2 Motivation and Goals $\quad 4$

2.1 The Standard Model and the Top Quark . . . . . . . . . . . . . . . . 4

2.2 New Physics Models and the Top Quark . . . . . . . . . . . . . . . 7

2.3 Current Status and Goals . . . . . . . . . . . . . . . . 7

3 Apparatus 10

3.1 Accelerator . . . . . . . . . . . . . . . . . . . 10

3.1 .1 Acceleration to $150 \mathrm{GeV} \ldots \ldots \ldots \ldots$

3.1.2 Anti-proton production .................. 11

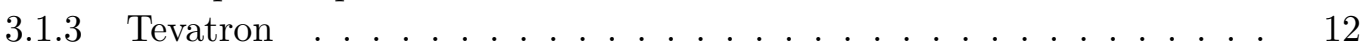

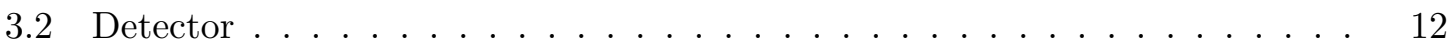

3.2.1 Tracking system . . . . . . . . . . . . . . . . 14

3.2 .2 Calorimeters . . . . . . . . . . . . . . . . . . . . 17

3.2 .3 Muon chambers . . . . . . . . . . . . . . . . . . 18

3.2 .4 Trigger and data acquisition . . . . . . . . . . . . . . . . 18

3.3 Software . . . . . . . . . . . . . . . . . . . . 19

3.3 .1 Calibrations . . . . . . . . . . . . . . . . . 19

3.3.2 Basic Reconstruction . . . . . . . . . . . . . . . . . . . 19

3.3.3 Monte Carlo generation and detector simulation . . . . . . . . 21

4 Selecting $t \bar{t}$ Candidate Events $\quad \mathbf{2 3}$

4.1 The $t \bar{t}$ Event Signature . . . . . . . . . . . . . . . . . . . . . 23

4.2 Particle Identification . . . . . . . . . . . . . . . . . . 24

4.3 Event Selection . . . . . . . . . . . . . . . . . . . . . . . . . . . . . . . . . . . . . . . . . . . . .

4.4 Expected Backgrounds . . . . . . . . . . . . . . . . 31 
5 Top Mass Reconstruction 33

5.1 Correcting Measured Quantities . . . . . . . . . . . . . . . . . . 33

5.1.1 Lepton corrections . . . . . . . . . . . . . . . . . . . 34

5.1.2 Generic jet corrections . . . . . . . . . . . . . . . . . . 34

5.1.3 Top-specific jet corrections . . . . . . . . . . . . . . . . 36

5.1.4 Additional jets, unclustered energy, and missing energy . . . . . . . 40

5.2 MINUIT fit . . . . . . . . . . . . . . . . . . . . . . 41

6 Extracting the Top Mass 47

6.1 Monte Carlo Signal Templates . . . . . . . . . . . . . . . . . . . . . . . . . 47

6.2 Monte Carlo Background Template . . . . . . . . . . . . . . . . . . . . 50

6.3 Likelihood . . . . . . . . . . . . . . . . . . . . . . . . . . 56

6.4 Likelihood Checks and Statistical Uncertainty . . . . . . . . . . . . . 58

7 Results from the data $\quad 61$

8 Systematic Uncertainties $\quad \mathbf{6 6}$

8.1 Jet Energy Scale and Jet Fragmentation . . . . . . . . . . . . . . . . . . . 67

8.1.1 Uncertainties on the jet energy . . . . . . . . . . . . . . . 67

8.1.2 Uncertainties on the top mass due to jet energy systematics . . . . . 68

8.2 Generators .. . . . . . . . . . . . . . . . . . . . 70

8.3 Initial State and Final State Radiation . . . . . . . . . . . . . . . . . . . . 72

8.4 Parton Distribution Functions . . . . . . . . . . . . . . . . . . . . . 72

8.5 Jet Resolution . . . . . . . . . . . . . . . . . . . . . . . . . 76

8.6 Top $p_{T}$ Distribution . . . . . . . . . . . . . . . . . . . . . . . . . 77

8.7 Background Shape .. . . . . . . . . . . . . . . . . . . 77

8.8 SecVtx $b$ Tagging . . . . . . . . . . . . . . . . . . . . . . . . . 79

8.9 Additional MC Modeling Checks . . . . . . . . . . . . . . . . . . . . . . 80

8.10 Summary of Systematic Uncertainties . . . . . . . . . . . . . . . 80

9 Other Checks $18 \mathbf{8 2}$

9.1 Subdivision of Top Mass Sample . . . . . . . . . . . . . . . . . . . . 82

9.2 Alternate Top-Specific Corrections . . . . . . . . . . . . . . . . . . . 83

$9.3 W$ Mass in the Hadronic Decay Mode . . . . . . . . . . . . . . . . . 86

9.4 Lepton $\eta$ Dependence . . . . . . . . . . . . . . . . . . . . . . . . . . . . . 86

9.5 Kinematic Distributions ......................... . 89

$9.6 \quad \chi^{2}$ Check .................................. 93

$\begin{array}{lr}10 \text { Conclusions } & 94\end{array}$

$\begin{array}{ll}\text { A Pseudoexperiments } & 95\end{array}$

$\begin{array}{ll}\text { B Pull Distributions and 68\% coverage } & 98\end{array}$

$\begin{array}{ll}\text { Bibliography } & 102\end{array}$ 


\section{List of Figures}

2.1 Loop diagrams for the $W$ propagator. . . . . . . . . . . . . . 5

2.2 Current top mass world average. . . . . . . . . . . . . 8

2.3 Top mass effect on Higgs mass prediction. . . . . . . . . . . . . . . 9

3.1 Components and layout of the Fermilab accelerator complex. . . . . . . . . 11

3.2 Accumulated luminosity delivered and recorded vs store number. . . . . . . 13

3.3 Elevation view of the CDF detector. . . . . . . . . . . . . . . . 14

3.4 Blowup of CDF tracking detectors . . . . . . . . . . . . . . 15

4.1 Diagram for $t \bar{t}$ production and decay. . . . . . . . . . . . . . . 24

4.2 Effect of the fourth jet energy cut on acceptance and reconstructed mass. . 28

5.1 Jet response before top-specific corrections. . . . . . . . . . . . . . . . 38

5.2 Reconstructed $W$ mass before and after top-specific corrections. . . . . . . . 39

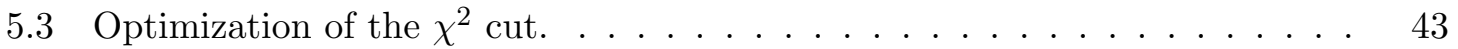

5.4 Effect of the $\chi^{2}$ cut on signal and background. . . . . . . . . . . 45

5.5 Reconstructed mass template for $175 \mathrm{GeV} / c^{2}$ generated mass. . . . . . . . 46

6.1 Signal mass templates with fitted parameterization overlaid. . . . . . . . . . 49

6.2 Mean, median, and most probable values of signal reconstructed mass as a function of generated mass. . . . . . . . . . . . . . . . . 50

6.3 Visualizations of the fitted probability density functions. . . . . . . . . 51

6.4 Comparison of $W+$ jets templates and data events with non-isolated leptons. $\quad 53$

6.5 Mass templates for different background processes. . . . . . . . . . . 55

6.6 Stacked reconstructed mass templates for background. . . . . . . . . . . . 57

6.7 Fitted top mass as a function of generated top mass (sanity check). . . . . . 59

6.8 Pull distribution centers and widths from pseudoexperiments. . . . . . . . 59

6.9 Estimated raw statistical uncertainties as a function of generated top mass. 60

7.1 Likelihood fit result, constrained and unconstrained fits. . . . . . . . . . 63

7.2 Positive and negative statistical uncertainties from pseudoexperiments. . . . 64

7.3 Distribution of positive vs negative statistical uncertainties from pseudoexperiments. . . . . . . . . . . . . . . . 64

7.4 Likelihood fit result for the 4-jet sample, constrained and unconstrained fits. 65 
8.1 Jet scale uncertainty as a function of $\Sigma E_{T}$ of 4 jets. Comparison of $\Sigma E_{T}$ for 3.5-jet and 4-jet samples. . . . . . . . . . . . . . 71

8.2 Integral, mean, median, and RMS for reweighted PDF templates. . . . . . . 74

8.3 Results of pseudoexperiments using reweighted PDF templates. . . . . . . . 75

8.4 Effects of jet energy smearing on jet response and reconstructed mass. . . . 78

9.1 Likelihood fit results when data sample is subdivided. . . . . . . . . . . . 84

9.2 Effect of using alternate top-specific corrections in pseudoexperiments. . . . 85

9.3 Effect of using alternate top-specific corrections on data events. . . . . . . . 87

9.4 Reconstructed mass of hadronic $W$ using jet assignments from the mass fitter.

Data and Monte Carlo are compared for $\geq 0, \geq 1$, and $2 b$ tags. . . . . . 88

9.5 Reconstructed mass distributions separated by type of primary lepton. . . . 89

9.6 Kinematic distributions: lepton $p_{T}$ and $\eta \ldots \ldots \ldots$. . . . . . . . . 90

9.7 Kinematic distributions: leading jet $E_{T}$ and $E_{T} \ldots \ldots \ldots \ldots$

9.8 Kinematic distributions: $H_{T}$ and number of jets. . . . . . . . . . . . 91

9.9 Kinematic distributions: $t / \bar{t} p_{T}$ and $\eta \ldots \ldots \ldots \ldots$. . . . . . . 91

9.10 Kinematic distributions: $p_{T}$ of $t \bar{t}$ system with ISR on and with ISR off. . . 92

9.11 Kinematic distributions: mass of the $t \bar{t}$ system. . . . . . . . . . . . . . . 92

9.12 Comparison of $\chi_{\min }^{2}$ in Monte Carlo and data. . . . . . . . . . . . . 93

B.1 Pull distributions for high-statistics pseudoexperiments. . . . . . . . . . 100

B.2 Pull widths and coverage of errors from pseudoexperiments. . . . . . . . . . 101 


\section{List of Tables}

4.1 Triggers used in this analysis. . . . . . . . . . . . . . . . 29

4.2 Events found in each jet/tag bin. . . . . . . . . . . . . . . 30

4.3 Event details for 28 candidate events used in the fit. . . . . . . . . . . . 30

4.4 Event details for nine candidate events failing the $\chi_{\min }^{2}<9$ cut. . . . . . 31

4.5 Background composition by process. . . . . . . . . . . . . . 32

6.1 Fitted parameters for the signal p.d.f.'s. . . . . . . . . . . . . . . 51

6.2 Efficiency of the $\chi^{2}$ cut for background events. Background normalizations used in fit. . . . . . . . . . . . . . . . . . . . . 54

6.3 Fitted parameters for the background p.d.f. . . . . . . . . . 56

7.1 Summary of fit results. . . . . . . . . . . . . . . . . . 62

8.1 Jet energy systematics with $\eta$ dependence. . . . . . . . . . . . . 68

8.2 Systematic uncertainties due to jet energy systematics. . . . . . . . . . . . 69

8.3 Fitted mass shifts using various systematic samples. . . . . . . . . . . . 70

8.4 Jet resolutions from photon-jet samples. . . . . . . . . . . . . . . . 77

8.5 Fitted mass shifts when the background shape is varied. . . . . . . . . . 79

8.6 Fitted mass shifts using background samples generated with different $Q^{2}$. $\quad 80$

8.7 Systematic uncertainty summary. . . . . . . . . . . . . . 81

9.1 Statistical uncertainty from pseudoexperiments with subdivided samples. $\quad$. $\quad 83$

9.2 Results of fit to data when sample is subdivided in likelihood fit. . . . . . . 83 


\section{Acknowledgments}

My deepest debt of gratitude for the existence of this dissertation is without a doubt to Young-Kee Kim, an adviser without equal. She consistently pushed me to achieve more and better things, without ever asking more than I could give. She always had my best interests in mind, and I am sincerely thankful for her advice and friendship.

I also thank my family for their love and support throughout my grad school years. My wife and best friend Luz kept me company through many late nights, and sustained me through the ebbs and tides of my enthusiasm. My parents and siblings, who have lately suffered their share and more of slings and arrows, have maintained their interest and excitement in my progress, and I am happy to give them something to be proud of.

The list of my colleagues who have given me important ideas, good advice, and fruitful collaboration is too long to be exhaustive here. I benefitted greatly from the experience of Lina Galtieri, Weiming Yao, and Jeremy Lys, as well as good-natured competition from Igor Volobouev. George Velev was a worthy champion of "what we did in run I," and translated key pieces of analysis code into $\mathrm{C}++$. Jean-Francois Arguin, Pekka Sinervo, Adam Gibson, Un-ki Yang, Mel Shochet, and Jahred Adelman, along with Young-Kee, made a great team to work with, in concert a sounding board amplifying the thoughts and ideas of any one member.

I owe a lot to Profs. Collett, Cook, Stoneking, and especially John Brandenberger, my mentors at Lawrence University who taught me by example how to get excited about physics (even if they were wary of the bright lights and fast cars of high-energy experiments).

Finally, the other grad students and postdocs in the Berkeley CDF group were fun and stimulating to work with. I shared many discussions, lunches, and jokes with Greg, Adam, Henri, Jason, and Aaron, among others. I am grateful that, although this stage of my life and career is ending, the friendships cultivated during this time are not. 


\section{Chapter 1}

\section{Introduction}

"The mass of the top quark is one of the most important measurements that can currently be performed in particle physics." Is this a true statement, and why? It is often pointed out by aficionados that the pole mass of the top quark is already more precisely known than that of any other quark. ${ }^{1}$ Yet the theoretical physics community clamors for more precise top mass estimates, to better than $1 \%$ relative uncertainty, while no such hunger manifests itself for, say, the charm quark mass.

The answer, of course, lies in two features of a more precise knowledge of the top quark mass. The first is its interconnection with other measurements, parameters, and presumptive models and theories. Few parameters of physical models are interesting in their own right, as numbers. ${ }^{2}$ But in the Standard Model of particle physics, the top quark mass is intimately related to various other parameters, including the mass of the putative Higgs particle (which has not yet been shown to exist, and in fact has been ruled out for certain values of its mass). Since the top quark mass, along with the $W$ boson mass, is the least well known of these parameters in terms of its effect in placing bounds on the Higgs mass, we say it is "poorly known," and we would like to measure it more precisely. In addition, the top quark mass enters into various alternative models of particle physics, and a more precise measurement can be used to further refine or reject many of those theories.

The second reason it is appealing to more precisely measure the top quark mass is that such a measurement is possible, in practical terms. The Tevatron accelerator at

\footnotetext{
${ }^{1}$ In terms of fractional uncertainty.

${ }^{2}$ Exception: numerical values are interesting when they happen to be $0,1, e$, or $\pi$. Indeed, another tantalizing feature of the top quark mass, when considered in terms of the dimensionless coupling constant to the Higgs field in the Standard Model, is that it is close to unity.
} 
Fermilab is churning out top quarks, and with the machine and detector upgrades completed for the current run, a larger sample of top-quark events is available for analysis than ever before.

This document will report a measurement of the top quark mass in the lepton + jets decay channel using the CDF detector at Fermilab, with $162 \mathrm{pb}^{-1}$ of collision data collected in 2002-2003 (run II). The method used is similar in spirit to an analysis performed at CDF using data from the 1995-1996 run (run I) [1]. Combined with other analyses that use more sophisticated techniques, this approach has several advantages. First, many parts of the detector, as well as all of the offline software, are new with respect to run I. By performing an analysis similar to the one published at the end of run I, we can draw on the great experience and expertise developed in run I and gain confidence in our run II tools and methods. Second, this analysis is based on kinematic reconstruction of the top mass, and as such makes fewer assumptions about the top quark production and decay mechanisms than other methods. Finally, this measurement will eventually be combined with measurements using the other techniques, so that we can take full advantage of non-overlapping statistical and systematic uncertainties.

A brief overview of the analysis is as follows. We scrutinize the data for events where a $t \bar{t}$ pair has been produced and has decayed to two $W$ bosons and two $b$ quarks, where subsequently one $W$ boson decayed to two quarks, and the other $W$ boson decayed to an electron or muon and a neutrino. Thus we look for a high-energy electron or muon, missing energy representing the neutrino, two jets of particles corresponding to the $b$ quarks, and two additional jets corresponding to the hadronic $W$ decay.

We will determine the mass of the top quark by comparing the distribution of some quantity from those data events with the shapes of the same quantity derived from events randomly generated and simulated at various values of the top mass. We also simulate events from the expected background processes. The top quark mass whose simulated events, when combined with the background, best describe the distribution in the data, is our measured value.

For the variable to be compared between data and simulated events, we choose a quantity that depends strongly on the mass of the top quark, thus affording good sensitivity. We choose the reconstructed top quark mass, determined in each event by minimizing a $\chi^{2}$ expression for the resolutions and kinematic relationships in the $t \bar{t}$ system. The mass reconstruction is complicated by the fact that we don't know which of the jets of particles 
to associate with each quark in the event. However, since we know something about the intermediate states of the system, such as the $W$ mass, the system is overconstrained, and we can choose which jet to assign to which quark based on its fit quality.

We will begin in the next chapter with a discussion of the theoretical framework for this measurement, elaborating on the motivations mentioned in this introduction. Next we will briefly describe the vast apparatus used to collect the relevant data. We will proceed with a detailed explanation of the analysis, and present the results obtained from the current dataset. Systematic errors will be enumerated and evaluated, and we will close by describing several crosschecks on our method and dataset. 


\section{Chapter 2}

\section{Motivation and Goals}

There are several good overviews of top quark phenomenology and related experimental questions and issues. References $[2,3,4]$ have been particularly useful.

\subsection{The Standard Model and the Top Quark}

Precision measurements in particle physics are carried out in the framework of the Standard Model, which has proved to be remarkably robust, despite decades of experiments searching for chinks in its now substantial armor.

The Standard Model describes the known fundamental particles and their interactions, apart from gravity. In the Standard Model there are three generations of fermions (spin- $\frac{1}{2}$ particles). Each generation consists of a pair of leptons, e.g. $e$ and $\nu_{e}$, whose interactions are governed by the electroweak forces, and a quark doublet, e.g. $u$ and $d$, which are subject to both electroweak and strong (QCD) forces. Bosons (spin-1 particles) are the force carriers, with the $\gamma, W^{ \pm}$, and $Z^{0}$ corresponding to the electroweak forces, and the gluon to the strong force.

The top quark $(t)$ is the third-generation quark with charge $+\frac{2}{3} e$, paired with the bottom quark $(b)$ in the weak interaction. It was discovered in 1995 at the Tevatron by both the CDF and D0 experiments [5,6], and so far has accorded with Standard Model predictions in its production rate, its decay channels, and various other properties. The greatest surprise has been its mass, which is not predicted by the Standard Model, but is a parameter of the model. With measurements so far indicating a mass of $170-185 \mathrm{GeV} / \mathrm{c}^{2}$, the top quark is by far the heaviest Standard Model particle, and is orders of magnitude 

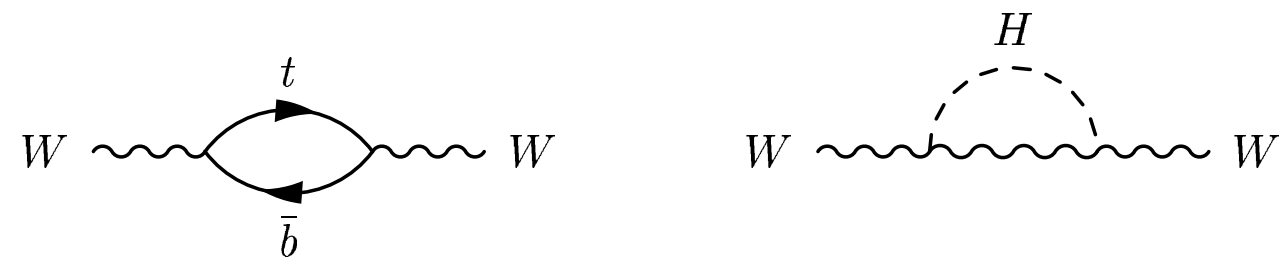

Figure 2.1: Loop diagrams generating corrections to the $W$ boson observed mass. On the left is a fermion loop with the top and bottom quarks. The correction from this diagram is proportional to $m_{t}^{2}$. On the right is a Higgs loop. This diagram contributes a correction proportional to $\ln m_{H}$.

more massive than the other fermions (the $b$ quark has a mass of roughly $5 \mathrm{GeV} / c^{2}$ ).

An important feature of the Standard Model is that it unifies the weak force with the electromagnetic force under the description of a single gauge theory, and in doing so explains how the $W$ and $Z$ bosons acquire mass. The electroweak gauge symmetry is spontaneously broken by interaction with a scalar field, the Higgs field. Although this model, proposed by Glashow, Weinberg, and Salam, is supported by all experimental observations to date, the Higgs particle associated with the Higgs field has yet to be discovered. To find this Higgs particle, or to rule it out (for there are competing explanations for the electroweak symmetry breaking that provides masses for the weak vector bosons), is the current holy grail of experimental particle physics. As it turns out, the mass of the top quark plays a key role in that quest.

In its simplest form, that found in the Standard Model, a Higgs particle of mass $m_{H}<114 \mathrm{GeV} / c^{2}$ has been ruled out by direct searches at LEP experiments. ${ }^{1}$ On the other hand, the Higgs mass can be constrained indirectly by virtue of its effect on the various observables of the Standard Model through radiative corrections, or loop effects.

Figure 2.1 shows how the Higgs can affect a particular observable, the $W$ mass, through a loop in the $W$ propagator. The same figure shows a top/bottom quark loop in the $W$ propagator, which similarly affects the observed $W$ mass. For example, in the on-shell renormalization scheme, the radiative corrections are encapsulated in the quantity $\Delta r$, where

$$
m_{W}=\frac{A_{0}}{\sin \theta_{W}(1-\Delta r)^{1 / 2}},
$$

\footnotetext{
${ }^{1}$ The Large Electron Positron collider at CERN was an $e^{+} e^{-}$accelerator operating at center-of-mass energies up to $212 \mathrm{GeV} / c^{2}$.
} 
and

$$
\Delta r \sim \Delta r_{0}-\frac{\rho_{t}}{\tan ^{2} \theta_{W}}
$$

The latter equation gives the leading contributions to the radiative corrections. $\Delta r_{0}$ (about $6 \%$ ) is related to the running of the fine structure constant $\alpha$, while $\rho_{t} / \tan ^{2} \theta_{W}$ is about $3 \%$, and has a quadratic dependence on the top mass:

$$
\rho_{t}=0.99 \% \cdot\left(\frac{m_{t}}{177.9 \mathrm{GeV} / c^{2}}\right)^{2} .
$$

In contrast, $\Delta r$ gets a smaller correction from the Higgs loop, and in addition, the Higgs mass enters only as a logarithm: $\Delta r_{H} \propto \ln m_{H}$. Thus, even if the $W$ mass is precisely measured, the Higgs mass cannot be well constrained based on its contribution to the observed $W$ mass unless the top quark mass is also very precisely known.

The analysis above pertains only to an observation of the $W$ mass, but the conclusions extrapolate. A complicated analysis of the relationships between electroweak observables (including the top mass) and the predicted radiative corrections, a sort of global consistency check, allows one to constrain the mass of the Standard Model Higgs particle [7], and the constraint is highly dependent on the top quark mass measurement and its uncertainty.

Another aspect of the top quark mass is that current measurements imply a dimensionless parameter of the Standard Model consistent with one. In the Standard Model, the Higgs boson generates fermion masses. A Yukawa coupling $\lambda$ to each fermion is allowed, which in the case of the top quark enters the SM Lagrangian as

$$
\Delta \mathcal{L}_{t}=\frac{1}{\sqrt{2}} \lambda_{t} v \bar{t}_{\mathrm{L}} t_{\mathrm{R}}+\text { h.c. },
$$

where $v$ is the vacuum expectation value of the Higgs boson. From this term the top quark mass can be read off:

$$
m_{t}=\frac{1}{\sqrt{2}} \lambda_{t} v
$$

Since the Higgs vacuum expectation value is known from electroweak measurements to be $v=246 \mathrm{GeV}$, we find

$$
\lambda_{t}=\frac{m_{t}}{173.9 \mathrm{GeV} / c^{2}} .
$$

Thus the Yukawa coupling of the top quark to the Higgs boson is very close to unity. This could be a coincidence, or it could have deeper meaning, signifying a special status for the top quark among known particles. 


\subsection{New Physics Models and the Top Quark}

The top quark often plays an important role in alternative explanations of elec-

troweak symmetry breaking [8]. One example is technicolor and related theories of dynamical electroweak symmetry breaking. In those theories the scalar field is not the Higgs but fermion condensates under a new strong-like gauge force that is present at the $\mathrm{TeV}$ energy scale.

Exotic physics models will not be confirmed or rejected based on a simple direct measurement of the top quark mass. On the other hand, all models must explain the same precision electroweak data, so more precise measurements can constrain or rule out the exotic models as well as the Standard Model. Models in which the top quark is involved in electroweak symmetry breaking will probably be even more sensitive to the top quark mass in the precision electroweak fits than is the Standard Model.

\subsection{Current Status and Goals}

The current world average of top mass measurements, using run I analyses from $\mathrm{CDF}$ and D0, is $178.0 \pm 4.3 \mathrm{GeV} / c^{2}$, as shown in Figure 2.2. This includes a contribution of $176.1 \pm 5.1$ (stat.) \pm 5.3 (syst.) $\mathrm{GeV} / c^{2}$ from the CDF run I lepton + jets channel measurement. One goal of this first run II measurement is to achieve a comparable uncertainty.

The current status of the precision electroweak fits is shown in Figure 2.3. We need to determine the precision we would like to achieve with existing experiments in order to best predict the Higgs mass, or to best test the Standard Model if the Higgs is observed. Let us assume the $W$ mass is measured at run II to an uncertainty of $20 \mathrm{MeV} / c^{2}$; then the precision needed on the top mass such that it does not dominate the uncertainty on the fitted Higgs mass is about $3 \mathrm{GeV} / c^{2}$. Thus $3 \mathrm{GeV} / c^{2}$ should be our goal for the top mass precision in run II combined across channels and experiments. 


\section{Top-Quark Mass [GeV]}

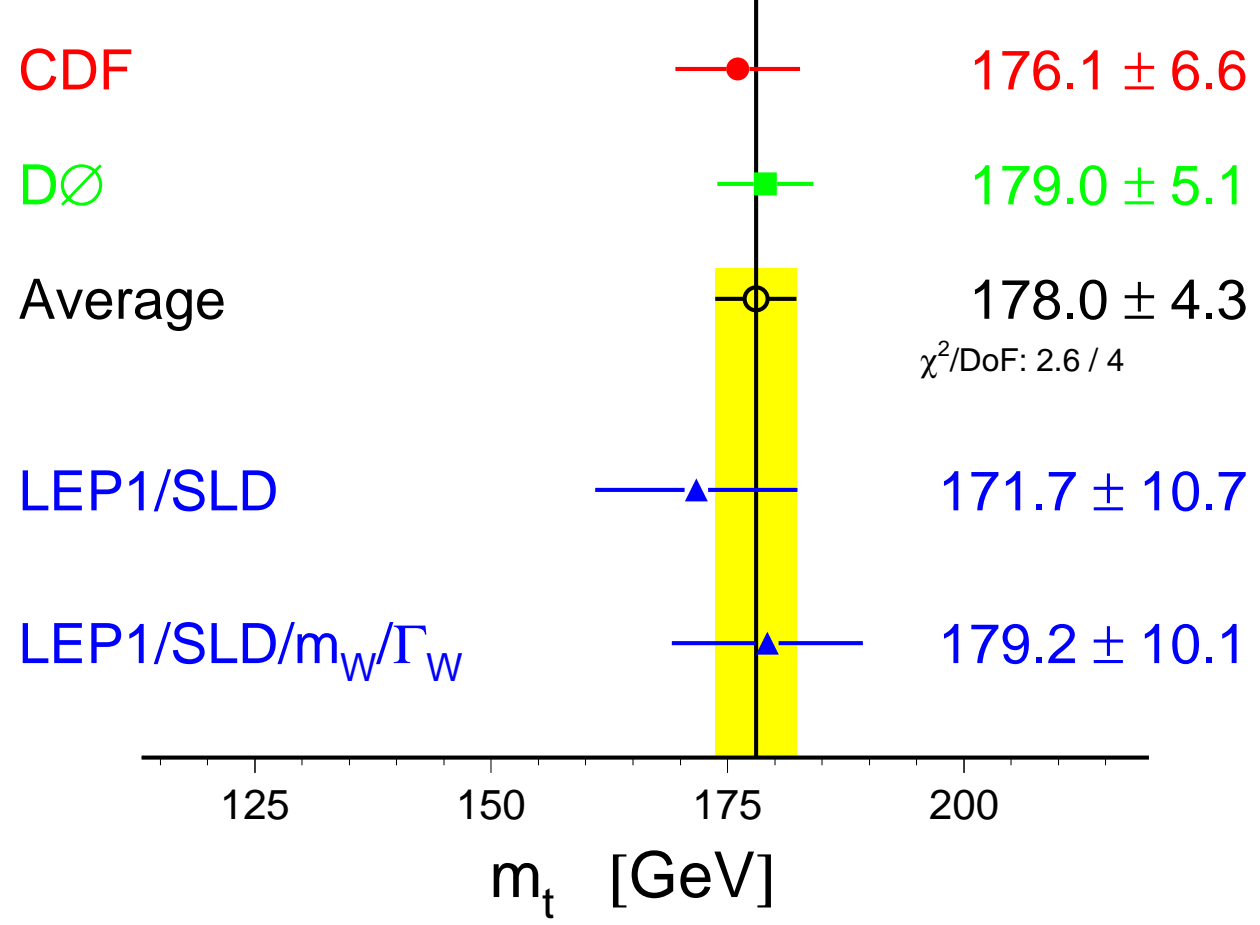

Plot courtesy of the LEP Electroweak Working Group (August 2004).

Figure 2.2: Top mass measurements from CDF and D0 in run I are shown, along with their combination (labeled "average"). Also shown are the Standard Model predictions based on other precision electroweak measurements. 

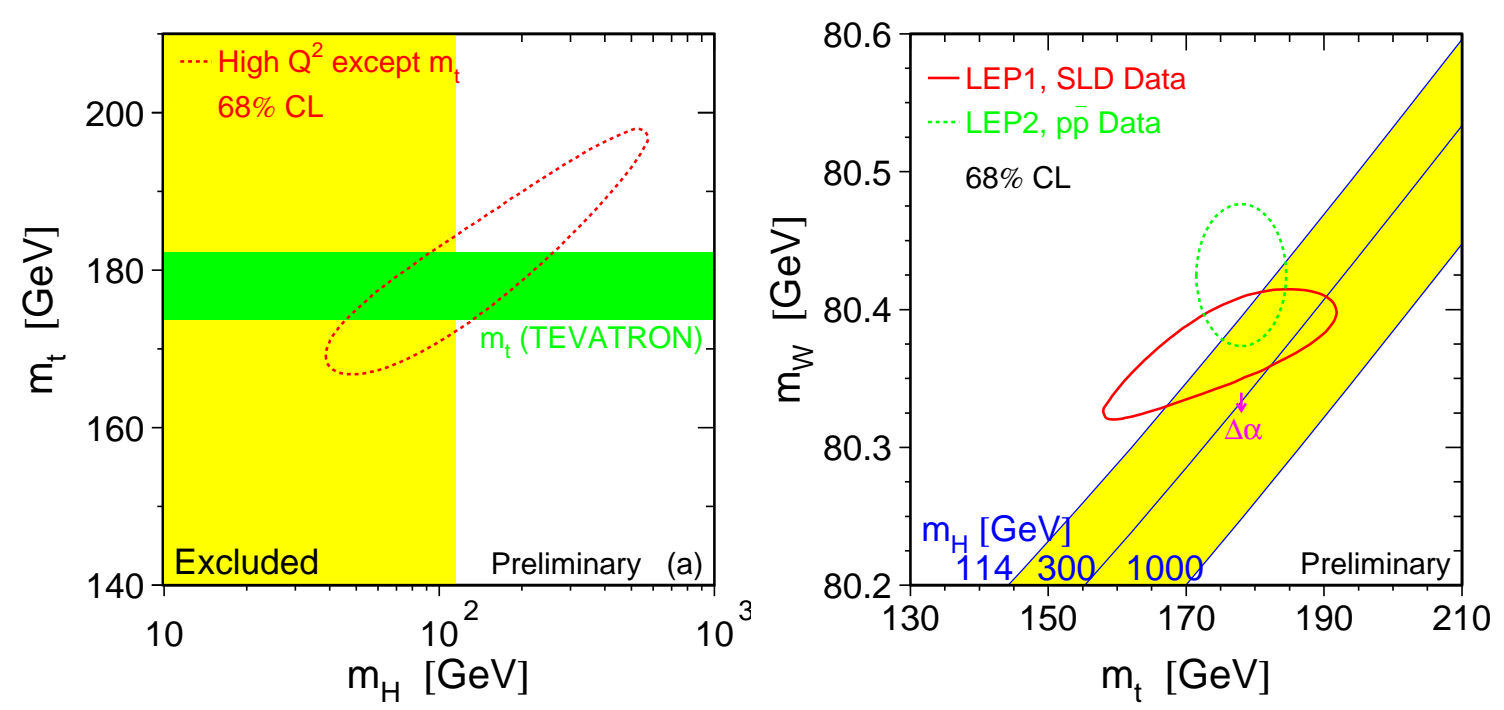

Plots courtesy of the LEP Electroweak Working Group (August 2004).

Figure 2.3: The relationship between the measured top mass and the predicted Standard Model Higgs mass, resulting from a fit to the body of precision electroweak measurements performed at LEP and SLD. On the left, the oval shows the allowed region (at $68 \%$ confidence level) in the top mass-Higgs mass plane. The yellow (light shaded) region is excluded by the unsuccessful direct Higgs searches at LEP, and the green (dark shaded) region is the current world average measurement of the top mass. On the right, the red (dark) oval shows the allowed region in the $W$ mass-top mass plane, while the green (light) oval shows the direct measurements of $m_{W}$ and $m_{t}$. The diagonal Higgs isomass lines refer to the best fit Higgs mass given $m_{W}$ and $m_{t}$. In both plots it can be seen that the preferred Higgs mass is close to the lower limit from direct searches. 


\section{Chapter 3}

\section{Apparatus}

The experimental apparatus used to perform the analysis described here is a complicated piece of equipment, whose design, construction, and operation have required vast sums of money and countless years of work by dedicated physicists, engineers, technicians, and others. It can generally be divided into three components. First is the accelerator, which causes protons and anti-protons to collide at a high center-of-mass energy and with great frequency at a very precise location. Second is the detector, which selects the collisions with the most interesting physics and records as much information as possible about the particles they produce. Last is the detector's offline software, which processes raw detector information and identifies useful physics objects as a prelude to all physics analyses. These three pieces are described below.

\subsection{Accelerator}

The accelerator complex at Fermilab comprises several machines, each of which contributes to the final product of $p \bar{p}$ collisions at $\sqrt{s}=1960 \mathrm{GeV}$. An overview of the system is shown in Figure 3.1. The Recycler has not contributed to accelerator operations during the collection of data used in this analysis, and it will not be described here.

\subsubsection{Acceleration to $150 \mathrm{GeV}$}

The first stage of particle acceleration at Fermilab is a Cockcroft-Walton accelerator, which is a DC machine that simply "drops" particles from a potential height of $750 \mathrm{keV}$. From there, the particles go through a linear accelerator, about $130 \mathrm{~m}$ long, that 


\section{FERMILAB'S ACCELERATOR CHAIN}

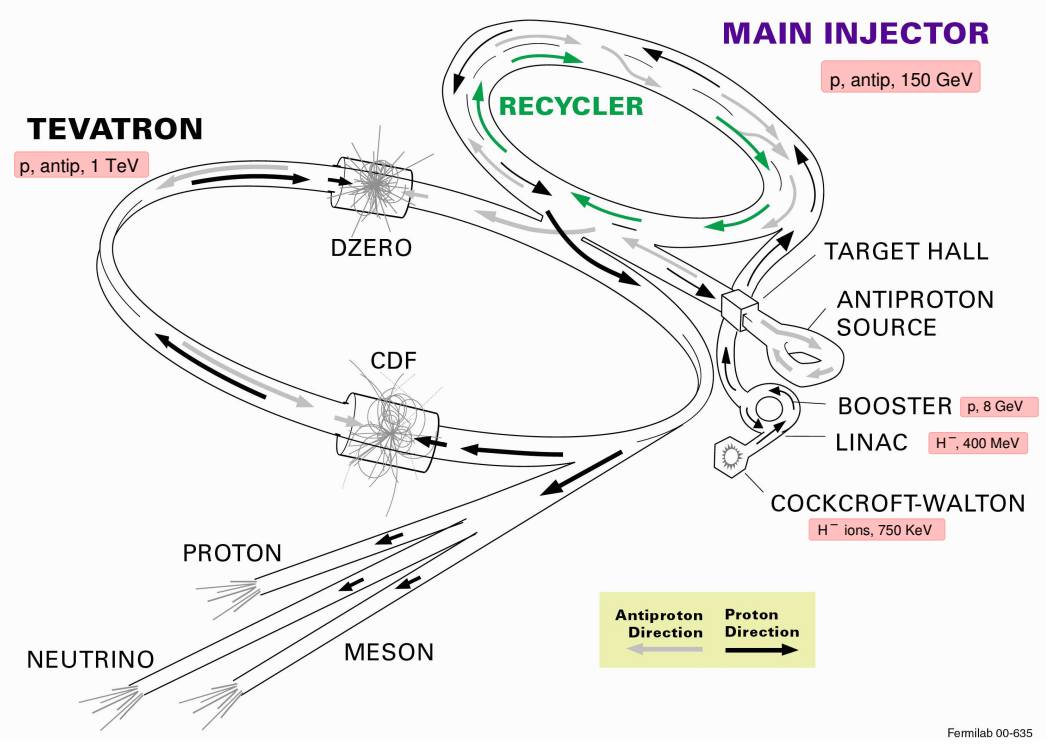

Figure 3.1: The components and layout of the Fermilab accelerator complex are shown. Black (gray) arrows indicate the direction of proton (anti-proton) beams. and labels give the particle type and maximum energy of each portion of the accelerator chain.

raises their energy to $400 \mathrm{MeV}$. The particles accelerated in these first stages are $\mathrm{H}^{-}$ions, i.e. hydrogen atoms with an extra electron. As the ions enter the next stage of the accelerator chain, called the Booster, their electrons are stripped off and what remain are bare protons. The Booster is a synchrotron accelerator with a circumference of $475 \mathrm{~m}$, which brings the proton energy up to $8 \mathrm{GeV}$.

From the booster, the $8 \mathrm{GeV}$ protons are sent to the Main Injector. This is a multipurpose synchrotron for both protons and anti-protons whose maximum energy is $150 \mathrm{GeV}$. Particles are sent from the Main Injector to the Tevatron for the final stage of acceleration and collisions. But first, the other half of $p \bar{p}$ collisions must be produced.

\subsubsection{Anti-proton production}

The protons (originally hydrogen ions) accelerated so far come from a simple can of compressed hydrogen gas. Anti-protons are not so easy to find - they must be painstakingly created, cooled, and stored. To do this, the Main Injector accelerates protons to $120 \mathrm{GeV}$ and sends them to the Anti-Proton Source. The protons smash into a nickel target and 
produce many particles, some of which are anti-protons that are selected using a bending magnet. The selected anti-protons are sent through the Debuncher, where they are cooled to form a beam with more uniform $8 \mathrm{GeV}$ energy, then to the Accumulator, where they are stored until enough anti-protons have been made to set up a new store in the Tevatron. The efficiency to get a stable anti-proton in the Accumulator from each proton sent to the anti-proton source is about $2 \times 10^{-5}$, so it can take many hours of "stacking" anti-protons to prepare enough for a store. Finally, the accumulated anti-protons are sent to the Main Injector to be accelerated to $150 \mathrm{GeV}$ and then injected into the Tevatron.

\subsubsection{Tevatron}

The final stage in the accelerating chain is the Tevatron itself, a synchrotron built using superconducting magnets with a bending magnetic field of $4.2 \mathrm{~T}$. The Tevatron accelerates both protons and anti-protons to $980 \mathrm{GeV}$ in opposing paths around the $1 \mathrm{~km}$ radius ring. At two predetermined points on the ring, where the CDF and D0 detectors are located, the beams are focused and steered into each other and particle collisions are produced. There are 36 bunches of protons and 36 bunches of anti-protons in the Tevatron, and bunch crossings occur every $396 \mathrm{~ns}$. The total zero bias rate is $1.7 \mathrm{MHz}{ }^{1}$

The accelerator performance was somewhat shaky at the beginning of run II, but recently significant improvements in the luminosity ${ }^{2}$ have been made. Figure 3.2 shows the integrated luminosity delivered by the accelerator and recorded by CDF. The current analysis uses data that has been calibrated and validated between March 2002, when the detector was fully commissioned, and September 2003, the most recent data for which all checks and calibrations have been performed.

\subsection{Detector}

The CDF detector is built on a principle common to most collider detectors: measure as many properties as possible of as many particles as possible. In particular, we would like to know the particle type and momentum four-vector of every particle produced in a given collision. Of course, in practice, not all the desired information can be measured.

\footnotetext{
${ }^{1}$ Not all bunches have protons and anti-protons, so this number is less than $\frac{1}{396 \mathrm{~ns}}$.

${ }^{2}$ The frequency of events of a given type is proportional to the instantaneous luminosity $(L)$ provided by the accelerator. The expectation value of number of events of a given type in a data sample is proportional to the integrated luminosity $\left(\mathcal{L}=\int L d t\right)$ for that sample.
} 


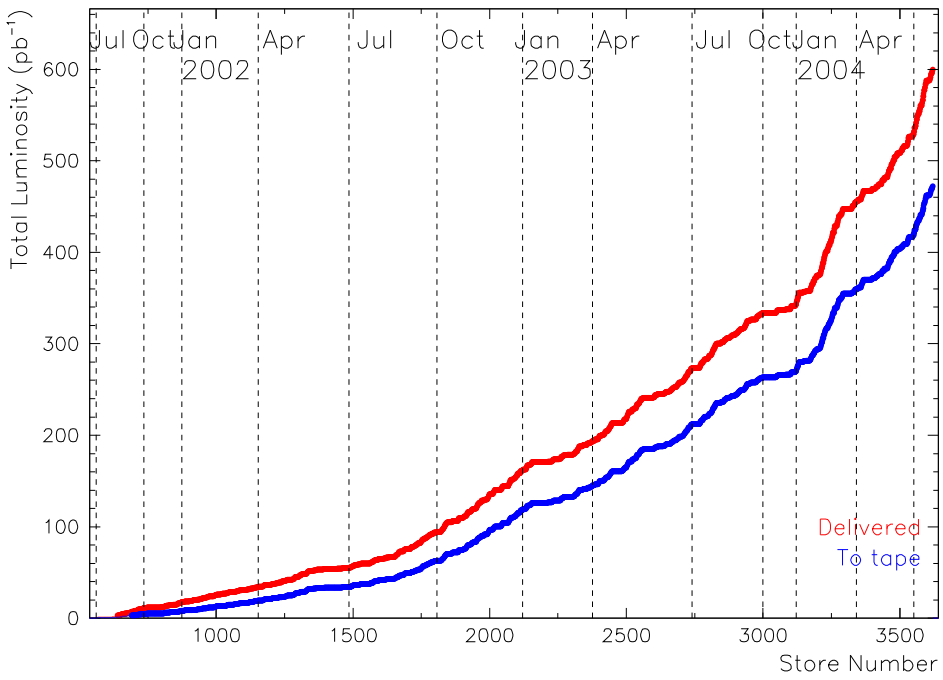

Figure 3.2: The accumulated luminosity delivered by the accelerator (upper curve) and recorded by CDF (lower curve) vs Tevatron store number. Rough time markers are given by the vertical dotted lines. This analysis uses data collected from March 2002 to September 2003.

Some particles are not measured at all, for example if they travel down the beampipe, where the detector is not instrumented. Other particles are not energetic enough to be reliably measured by the detector. Some particles may overlap in the detector, so that inferring their individual properties is difficult or impossible.

CDF is composed of several subsystems, which are responsible for different types of measurements. The detector geometry is cylindrical, with the $z$ axis pointing along a tangent to the Tevatron ring, in the direction of proton flight in the accelerator. The $x$ axis points outward from the accelerator ring, and the $y$ axis points straight up. The angle $\theta$ is the polar angle measured from the proton direction, and $\eta=-\frac{1}{2} \ln (\tan \theta)$ is the pseudorapidity, ${ }^{3}$ a useful coordinate at a hadron collider since the difference $\Delta \eta=\eta_{1}-\eta_{2}$ is invariant under Lorentz boosts along the $z$ axis. Typically by $\eta$ we refer to the detector $\eta$, which is calculated assuming an origin of $(0,0,0)$. But $\eta$ can also refer to "event $\eta$ ", where the origin of the coordinate system is the interaction point.

Overall, the detector measures about $15 \mathrm{~m}$ in length, and about $10 \mathrm{~m}$ in diameter.

\footnotetext{
${ }^{3}$ Pseudorapidity is equal to rapidity, $y=\frac{1}{2} \ln \frac{E+p_{z}}{E-p_{z}}$, for high-momentum particles.
} 


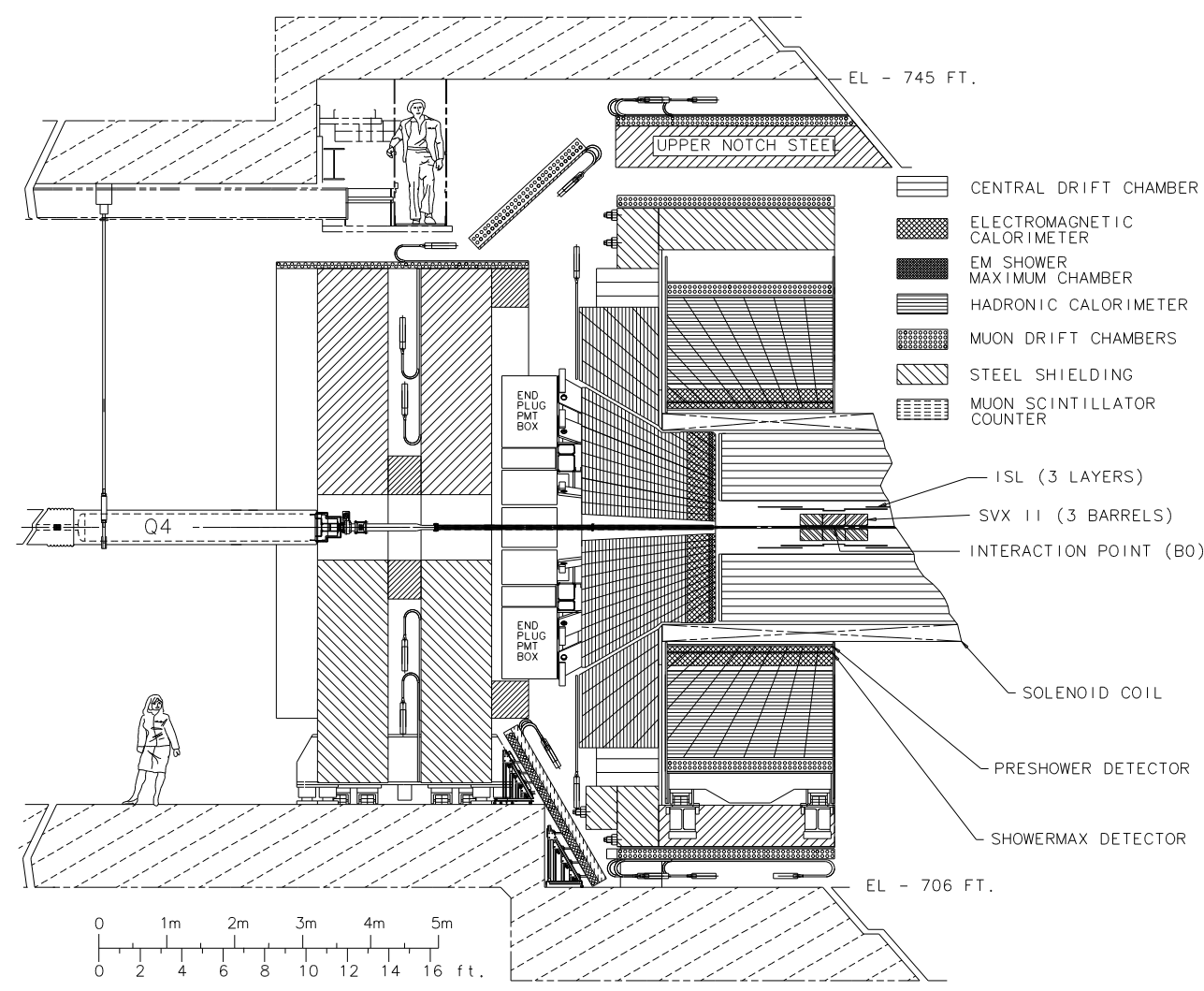

Figure 3.3: This elevation view of CDF shows the onion-like nature of the typical particle physics detector. The subdetectors are concentric, each covering as much solid angle as possible around the interaction point, and each with a roughly cylindrical geometry. From inside to outside, CDF features a silicon strip detector, a tracking drift chamber, an electromagnetic calorimeter, a hadronic calorimeter, and muon chambers.

The subdetectors are arranged in cylindrical shells, so that a given particle in principle traverses each detector (until it decays in flight or stops, for example in the calorimeter). Figure 3.3 shows a schematic of the CDF detector. By the "central" region, we mean roughly $|\eta| \leq 1.0$. The "plug" or "forward" region is $1.0 \leq|\eta| \leq 3.6$.

In the following sections, the subdetectors important to this analysis are described in more detail. Many smaller systems, as well as those less immediately relevant, have been left out.

\subsubsection{Tracking system}

The CDF tracking system is the first thing seen by a particle leaving the interaction point and exiting the beampipe. The aim of the tracking detectors is to measure the 


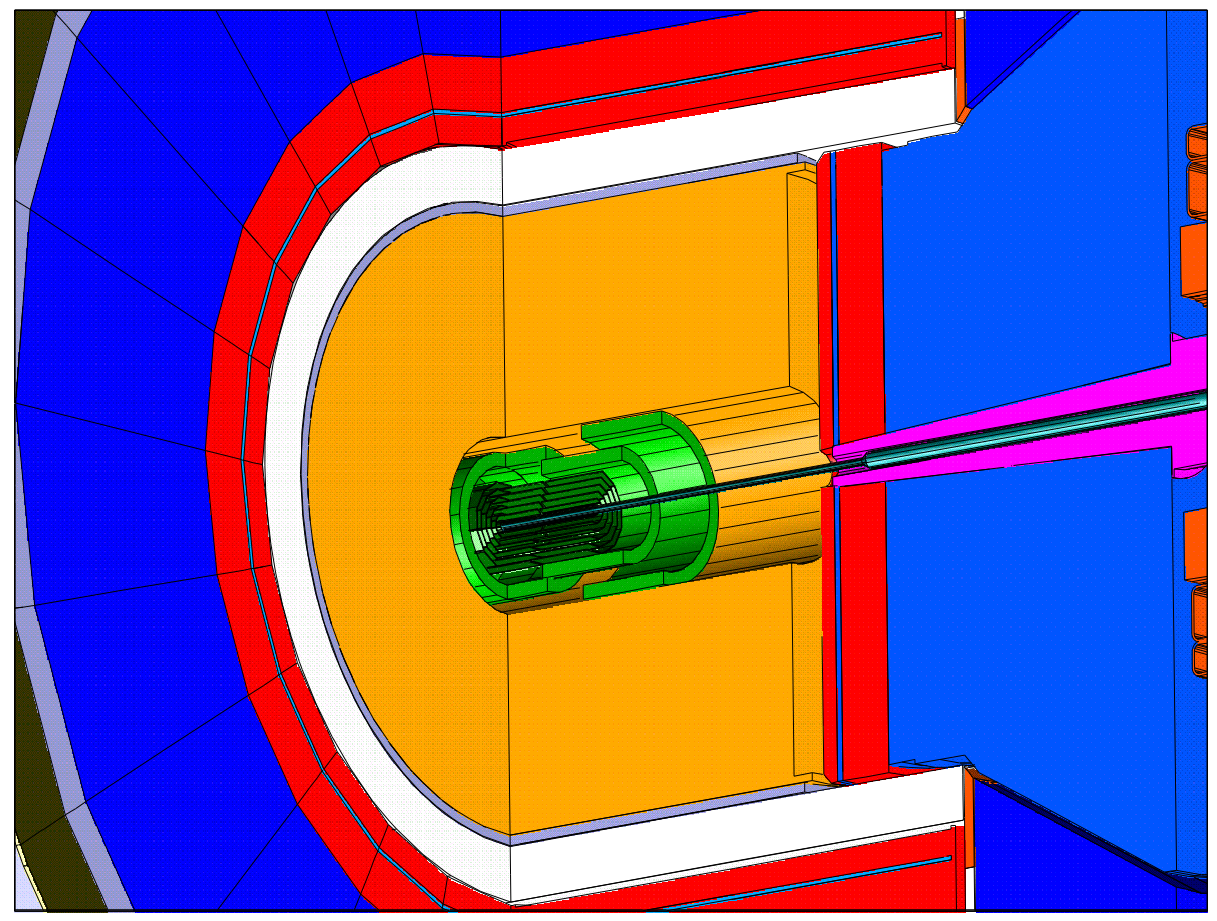

Figure 3.4: This is a zoomed view of the CDF tracking detectors, which are surrounded by a solenoid (white) providing a magnetic field of $1.4 \mathrm{~T}$. At the center is the silicon strip detector with eight layers of silicon (green/dark), while between the silicon and the solenoid is a large drift chamber with 96 layers of sense wires (orange/light). Also visible outside the solenoid are the electromagnetic (red/dark) and hadronic (blue/darker) calorimeters.

momentum of charged particles by observing their curvature in a magnetic field. ${ }^{4}$ By combining the measurements of several such particles, we can also reconstruct vertices from which the particles originated, both the primary vertex where the interaction occurred, and any secondary vertices due to decays of long-lived particles. The measurements must be performed with very little perturbation of the particle itself. Figure 3.4 shows a cutaway view of the tracking system. The magnetic field of $1.4 \mathrm{~T}$ is provided by a superconducting solenoid surrounding the central drift chamber and silicon detectors.

\footnotetext{
${ }^{4}$ Neutral particles are not measured by the tracker, both because they don't curve in the magnetic field and because the position measurements in the tracking detectors typically involve ionization reactions.
} 


\section{Central Outer Tracker}

The Central Outer Tracker (COT) [9] measures particle locations over a large radial distance, so that a precise measurement of the curvature, and therefore momentum, can be made. It is a large open-cell drift chamber with 8 "superlayers" (4 axial and 4 with a $3^{\circ}$ stereo angle), each of which contains 12 wire layers, for a total of 96 layers. There are 30,240 wires in total. The COT occupies a volume $310 \mathrm{~cm}$ in length and between radii of $43 \mathrm{~cm}$ and $130 \mathrm{~cm}$.

\section{Silicon}

The silicon detector [10] provides position measurements with very high resolution for charged particles close to the interaction region. This is important in extrapolating tracks all the way back to the collision point and determining whether the particle came from the primary interaction or was produced at a secondary, displaced vertex, as from a decay in flight.

There are three separate silicon microstrip subdetectors, in which all but the innermost layer use double-sided wafers of silicon to get measurements in both axial and stereo directions for three-dimensional tracking. There are a total of 722,432 channels in eight layers. Typical strip pitch is $55-65 \mu \mathrm{m}$ for axial strips, $60-75 \mu \mathrm{m}$ for $1.2^{\circ}$ small-angle stereo strips, and $125-145 \mu \mathrm{m}$ for $90^{\circ}$ stereo strips.

The SVXII detector is $90 \mathrm{~cm}$ long and contains 12 wedges in $\phi$, each with 5 layers of silicon at radii from $2.5 \mathrm{~cm}$ to $10.6 \mathrm{~cm}$. One side of each layer contains strips oriented in the axial direction, and the other side contains $90^{\circ}$ stereo strips in three cases, and $1.2^{\circ}$ small-angle stereo strips in two cases. The Intermediate Silicon Layers (ISL) comprise two layers of silicon at larger radii $(20 \mathrm{~cm}$ and $28 \mathrm{~cm})$. Besides providing extra measurements between the COT and SVXII to ease track reconstruction in the central region, the ISL also allows tracks to be reconstructed at high $\eta$ with larger level arm, and therefore better momentum resolution, since COT coverage drops off in the region $1<|\eta|<2$. Finally, layer 00 (L00) is a layer of silicon mounted directly on the beampipe to provide one more measurement as close to the collision point as possible. 


\subsubsection{Calorimeters}

The task of the calorimeters is to measure the energy of particles that have a large cross-section for electromagnetic interactions (electrons, photons) or nuclear interactions (hadrons). CDF uses sampling calorimeters, which measure energy proportional to the total number of particles created in an electromagnetic or hadronic shower. The calorimeters are segmented into "towers" that have projective geometry, i.e. the divisions between towers always point directly at the nominal interaction point $(0,0,0)$. The segmentation of the CDF calorimeters is rather large, so that often several particles contribute to the energy measured in one tower.

\section{Central calorimeter}

In the central region, i.e. $|\eta|<1.1$, the calorimeter is divided into wedges subtending $15^{\circ}$ in $\phi$. Each wedge has ten towers, of roughly equal size in $\eta$, on each side of $\eta=0$.

The central electromagnetic calorimeter (CEM) [11] contains alternating layers of lead and scintillator, making 18 radiation lengths of material. The resolution for high-

energy electrons and photons is $\frac{\sigma\left(E_{T}\right)}{E_{T}}=13.5 \% / \sqrt{E_{T}} \oplus 1.5 \%$. Embedded in the CEM is a shower maximum detector, the CES, which provides good position measurements of EM showers and is used in electron identification. The CES consists of wire proportional chambers $1.45 \mathrm{~cm}$ wide, with cathode strips (1.67-2.01 cm wide) providing stereo position information.

The central hadronic calorimeter (CHA) and the end wall hadronic calorimeter (WHA) [12] are of similar construction, with alternating layers of steel and scintillator (4.7 interaction lengths). The WHA fills a gap in the projective geometry between the CHA and the plug calorimeter.

\section{Plug calorimeter}

The calorimetry in the end plugs $(|\eta|>1)$ is new for run II. In principle, the plug calorimeters [13] are similar to the central ones, but they use more modern technology. The tower geometry is more complicated in the plug, but the $15^{\circ}$ wedge pattern is respected.

The plug electromagnetic calorimeter (PEM) has lead absorber and scintillating tile read out with wavelength shifting fibers. An electron traversing the PEM sees 
23.2 radiation lengths. The resolution for high-energy electrons and photons is $\frac{\sigma(E)}{E}=$ $14.4 \% / \sqrt{E} \oplus 0.7 \%$. As in the central, there is a shower maximum detector (PES), which measures the position of electron and photon showers. The plug hadronic calorimeter (PHA) has alternating layers of iron and scintillating tile, for a total of 6.8 interaction lengths.

\subsubsection{Muon chambers}

Since muons do not have large cross-sections for either electromagnetic or nuclear interactions, they typically travel through the calorimeters intact, leaving only ionization energy behind (the so-called minimum ionizing particle, or MIP, energy). The presence of a muon can then be confirmed by hits in the muon chambers, which are banks of single-wire drift cells four layers deep.

There are three muon subdetectors used in this analysis. The CMU [14] is located directly behind the hadronic calorimeter in a limited portion of the central region $(|\eta|<0.6)$. The CMP adds additional coverage in the central region and reduces background with an additional $60 \mathrm{~cm}$ of steel shielding, corresponding to 2.4 interaction lengths at $90^{\circ}$. The CMX covers the region $0.6<|\eta|<1.0$, and contains eight layers of drift tubes, with the average muon passing through six.

\subsubsection{Trigger and data acquisition}

The Tevatron produces proton and anti-proton bunch crossings about 1.7 million times per second. The detector acquires data for each of those potential events, but that rate must be immediately reduced to the rate that $\mathrm{CDF}$ can write events to tape, about $75 \mathrm{~Hz}$, saving of course only the most interesting events. This is accomplished with a threelevel trigger system. This analysis uses data from triggers based on high- $p_{T}$ leptons, which

come from the leptonically decaying $W$ in the event. See Table 4.1 for the full trigger paths used in this analysis.

At the first level, a hardware trigger looks for signs of an interesting event, such as a calorimeter tower with large energy, or a stiff track. The level 1 trigger takes about $4 \mu \mathrm{s}$, and the accept rate is about $40 \mathrm{kHz}$. The extremely fast tracker (XFT) finds tracks using the four axial layers of the COT, with a momentum resolution of better than $2 \% / \mathrm{GeV} / c$.

The level 2 trigger is also a hardware trigger, and performs some basic reconstruction, such as simple calorimeter clustering, to reduce the event rate to about $300 \mathrm{~Hz}$. A 
silicon vertex trigger (SVT) also operates at level 2, but is not used in this analysis.

Finally, the level 3 trigger uses an array of hundreds of PCs to perform full software reconstruction of the remaining events. Even more stringent requirements are imposed to reduce the event rate to the allowed $75 \mathrm{~Hz}$.

\subsection{Software}

High-energy physics analyses are largely an exercise in data reduction, and that process begins and ends with computer software. CDF software is almost all written in $\mathrm{C}^{++}$, and is all new since run I. It uses a framework developed for particle physics experiments called $\mathrm{AC}^{++}$, which has a modular structure, so that pieces of code with different functionality can be added and removed depending on the task at hand.

\subsubsection{Calibrations}

After the raw data is read out from the detector and written to tape, the first step in making something intelligible of the data is to apply calibrations. These are typically derived in situ, by looking at large data samples and determining the average corrections needed. For example, calorimeter energies are corrected due to known differences in the response for different towers. These calibrations are obtained by looking at large samples of electrons and comparing their EM energy to track momentum, and by examining the MIP energy of muons passing through the hadronic towers. The tracking system measurements are modified to account for slight misalignments, again determined by looking at large samples of tracks and checking track residuals on the smallest physical detector units, such as a silicon wafer or a COT cell of 12 wires.

\subsubsection{Basic Reconstruction}

The next step is to reconstruct interesting objects from the raw data. Some important steps are described here in more detail.

\section{Tracking}

Track reconstruction, as used in this analysis, begins with the COT. In each superlayer, short stubs are formed from hits in the 12 layers, then the stubs are linked 
together to form tracks. For high- $p_{T}$ tracks from $W$ bosons fiducial to the COT, the tracking efficiency has been measured at $98.3 \%$, while the momentum resolution is about $\sigma_{p_{T}} / p_{T}^{2} \approx 1.5 \times 10^{-3} /[\mathrm{GeV} / c]$. When a COT track is forced to pass through the beamline, the lever arm is increased, and the momentum resolution is improved by a factor of two; this is called beam-constrained tracking.

Subsequently, the tracks (without beam constraint) are extended into the silicon region by looking for hits in each layer consistent with the existing COT track. Adding silicon hits also improves the momentum resolution by about a factor of two, and more importantly provides good pointing resolution, relevant for reconstructing vertices from multiple tracks. The resolution of the impact parameter, the distance of a track's closest approach to the nominal beamline, is about $40 \mu \mathrm{m}$, which includes the $\approx 30 \mu \mathrm{m}$ intrinsic width of the beam itself.

\section{Clustering}

Calorimeter clustering of several types is performed. Electrons deposit their energy in a small region, so as the first step in electron identification, two-tower clusters are formed from the electromagnetic towers in the central region. Clustering is not allowed across wedge $(\phi)$ boundaries, where a "crack" allows energy to escape detection.

Electron showers deliver their strongest signal about six radiation lengths into the electromagnetic calorimeter, where they pass through the CES shower maximum detector, leaving information about their position and profile. Clusters of nine strips and nine wires are formed, and the position of the electromagnetic shower is determined by a fit to the cluster shape.

Jet clustering for this analysis is performed by looking for clusters of energy in the calorimeter using a cone algorithm, JETCLU [15], where the cone radius is $\Delta R=$ $\sqrt{\Delta \eta_{\text {evt }}^{2}+\Delta \phi^{2}}=0.4$. High-energy towers are used as a seeds, then nearby towers are added to the clusters, out to the maximum radius of 0.4. A final step of splitting and merging is performed such that in the end a tower does not contribute to more than one jet. 


\section{Muon stub reconstruction}

Sets of hits consistent with a particle trajectory, called stubs, are found in three or four layers of the muon chambers. Each stub is fit to a straight line, and its position and direction are recorded. This information can later be compared with extrapolations of tracks in the tracking chamber to reconstruct a muon.

\section{Missing $E_{T}$}

Information from all of the calorimeter towers is combined in a quantity called missing transverse energy, or $\boldsymbol{E}_{T}$. The momentum vectors for each tower in the central, wall, and plug calorimeters are projected into the plane transverse to the beams and summed. The opposite of this two-dimensional vector sum is the missing $E_{T}: \vec{E}_{T}=-\sum_{\text {towers }} \vec{E}_{i} \sin \theta_{i}$. Studies of the missing $E_{T}$ in minimum bias events indicates a resolution of roughly 0.4 .

$\sqrt{\sum E_{T}}$ on each component of $\overrightarrow{E_{T}}$, where $\sum E_{T}$ is the scalar sum of the transverse energy of towers in the calorimeters. The quantity $\vec{E}_{T}$ can be thought of as momentum that escaped the detector, given a perfectly balanced collision, and is often interpreted as neutrino energy (see section 4.2). $E_{T}$ is typically used as a scalar, in which case it refers to the magnitude of the vector defined above.

\subsubsection{Monte Carlo generation and detector simulation}

This top mass analysis relies heavily on the use of Monte Carlo (MC) event generation and detector simulation. Since $t \bar{t}$ production and decay is a complicated process, with many final state objects, and since the CDF detector is immensely complicated, with efficiencies and resolutions that are not at all uniform or easy to parameterize, it is virtually impossible to analytically predict the distribution of complicated observables such as the reconstructed mass described in chapter 5. Instead, we use the process of event generation and simulation described here to produce simulated events that we can analyze in the same way as data to produce predicted distributions. Thus, since we would like to perform a precision measurement of the top quark mass by comparing data to MC predictions, we need a very reliable and accurate simulation, robust and quick enough to produce large numbers of events to compare with the data.

For event generation we use the HERWIG [16], Pythia [17], and ALPGEN [18] programs. The Monte Carlo approach means that, given a process to simulate, the details 
of the production, as well as decays and kinematics, are all treated randomly event by event, taking into account all the relative probabilities.

The detector simulation takes everything produced by the generator (often hundreds of final-state particles) and "swims" them through a detailed description of the CDF detector, simulating the behavior of each subdetector and filling data banks identical to those recorded by the actual detector. The GEANT package provides a good description of most interactions in the CDF detector. Detailed models are developed and tuned to describe, for example, the COT ionization and drift properties so that high-level quantities like tracking efficiency and momentum resolution from the data can be reproduced. The calorimeter simulation is performed using a parameterized shower simulation (GFLASH) tuned to single particle energy response and shower shape from the data. 


\section{Chapter 4}

\section{Selecting $t \bar{t}$ Candidate Events}

\subsection{The $t \bar{t}$ Event Signature}

At the Tevatron, most top quarks are produced in top-antitop pairs, through quark annihilation $(\sim 85 \%)$ and gluon fusion $(\sim 15 \%)$. The $t \bar{t}$ production rate has been measured in both run I and run II and is consistent with the Standard Model prediction [19]. In order to measure the top mass, we must first find the extremely rare $t \bar{t}$ events among the millions of events recorded to tape.

The Standard Model top quark decays into a $W$ boson and a $b$ quark, immediately $\left(\tau \approx 4 \times 10^{-25} \mathrm{~s}\right)$ and essentially $100 \%$ of the time. The $t \bar{t}$ event signature is therefore determined by the decay products of the two $W \mathrm{~s}$, each of which can produce two quarks or two leptons $\left(e \nu_{e}, \mu \nu_{\mu}\right.$, or $\left.\tau \nu_{\tau}\right)$. Figure 4.1 shows the diagram for a typical case of $t \bar{t}$ production through quark annihilation. When more leptons are present, it is easier to distinguish the event from the huge number of uninteresting jet-filled events produced at a $p \bar{p}$ collider. However, the dilepton channel, marked by decays of both $W$ bosons to $e$ or $\mu$ and neutrino, suffers from a low branching ratio $(\sim 5 \%)$, and from difficulties in mass reconstruction due to the presence of two undetected neutrinos. This analysis considers events in the "lepton + jets" channel, where one $W$ decays to quarks and the other $W$ decays to $e \nu_{e}$ or $\mu \nu_{\mu}{ }^{1}$

Thus interesting events have a high- $p_{T}$ isolated $e$ or $\mu$, a neutrino, and four jets, two of which are $b$ jets. More jets may be present due to hard gluon radiation from an

\footnotetext{
${ }^{1}$ Events where one $W$ decays to $\tau \nu_{\tau}$ are problematic. The $\tau$ is not observed directly, and its decay products always include at least one neutrino, posing challenges in both identification and mass reconstruction.
} 


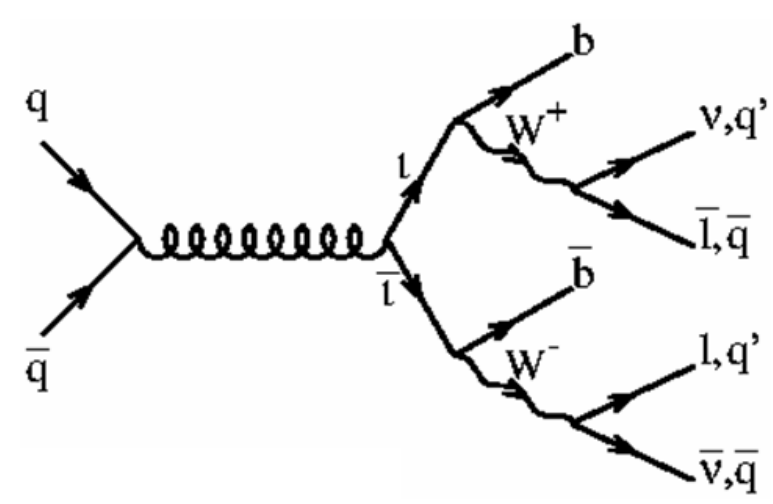

Figure 4.1: A typical diagram for $t \bar{t}$ production at the Tevatron (through quark annihilation) and the subsequent decay into $W^{+} W^{-} b \bar{b}$. The event signature is determined by the decays of the two $W$ bosons.

incoming parton (initial state radiation or ISR) or from a final-state quark (final state radiation or FSR).

There are several non- $t \bar{t}$ processes that have similar signatures and enter into the event sample for this analysis. Events where a leptonically decaying $W$ boson is found in association with QCD production of at least four additional jets, sometimes including a $b \bar{b}$ pair, have the same signature and are an irreducible background. Additional background events enter the sample when the $t \bar{t}$ signature is faked. For example, a jet can fake an isolated lepton with very low efficiency, a neutrino can be mistakenly inferred when the missing energy in the event is mismeasured, and a leptonically decaying $Z$ boson can look like a $W$ if one lepton goes undetected.

\subsection{Particle Identification}

Quarks, of course, even those with longer lifetimes than top, are not observed directly in the detector. In a process related to the QCD principle of asymptotic freedom, a quark "hadronizes" into a group of particles (hadrons), whose momentum vectors are clustered around the original flight path of the bare quark. These groups of particles are called jets. $^{2}$ See section 3.3.2 for jet clustering details. We identify each jet with a quark or gluon from the hard scattering interaction, but this is not always a robust assignment. Two quarks headed in roughly the same direction can produce overlapping or indistinguishable

\footnotetext{
${ }^{2}$ Gluons also produce jets by the same mechanism. For our purposes, quark jets and gluon jets are indistinguishable.
} 
clusters in the calorimeter. A single quark can emit a hard gluon before hadronization and form what appears to be two independent energy clusters. And in the end, even the theoretical assignment of a final-state particle to a bare quark is ambiguous, due to interactions among the original quarks and gluons during hadronization.

Jets produced by $b$ quarks ( $b$ jets) are particularly interesting objects. Since a top quark always decays into a $b$ jet, identifying $b$ jets in our sample is one way to distinguish $t \bar{t}$ signal events from background that does not prefer $b$ jets over other quark and gluon jets. We will also see that we can improve our jet energy resolution by applying different calibrations to $b$ jets (section 5.1.3). Experimentally, $b$ jets can be distinguished from their light quark counterparts because they always contain a $B$ meson or one of the baryons with $b$ content. These particles have relatively long lifetimes, on the order of $1.4 \mathrm{ps}$, so that they can travel millimeters from the interaction point before decaying. Thus jets can be identified as $b$ jets using a displaced vertex tagging algorithm, called SecVtx, which proceeds as follows. Jets with $E_{T}>15 \mathrm{GeV}$ are scanned for good-quality tracks with both COT and silicon information. When a secondary vertex can be reconstructed from at least two of those tracks, the distance between the primary and secondary vertices in the plane transverse to the beams $\left(L_{x y}\right)$ is calculated, along with its uncertainty $\left(\sigma\left(L_{x y}\right)\right)$. If $L_{x y} / \sigma\left(L_{x y}\right)>3$, the jet is considered tagged. The jet-by-jet $b$ tagging efficiency, when the jet is fiducial to the tracking region, is $40-50 \%$ in the typical energy range of $b$ jets from top decay. Overall for $t \bar{t}$ lepton + jets events, the efficiency for tagging at least one $b$ jet in an event is $54 \%$.

Since the energy required to produce the two tops $(\sim 350 \mathrm{GeV})$ is usually a large part of the center-of-mass energy of the colliding partons, $\sqrt{\hat{s}}$, they are produced at nonrelativistic velocities. ${ }^{3}$ This means that the decay products of a given top quark cannot be counted on to be near each other, and any top mass reconstruction must deal with the combinatoric problem of assigning jets to the partons from $t \bar{t}$ decay. When one or more jets are $b$ tagged, the number of possible jet-parton assignments is reduced.

High- $p_{T}$ isolated leptons ${ }^{4}$ and neutrinos are experimentally much less complicated objects than jets. Electrons are identified by a high-momentum track in the tracking detectors matched with an energy cluster in the electromagnetic calorimeter with $E_{T}>20 \mathrm{GeV}$.

\footnotetext{
${ }^{3}$ The center-of-mass energy of the $p \bar{p}$ system is $\sqrt{s}=1960 \mathrm{GeV}$, but each colliding parton carries only a fraction of the total proton momentum.

${ }^{4}$ From now on, we mean by "lepton" the electron or muon from the leptonically decaying $W$, although technically the word could refer to a tau or any neutrino as well.
} 
Photon and hadronic fakes are reduced by requiring the ratio of calorimeter energy to track momentum to be no greater than $2\left(\right.$ unless $\left.p_{T}>50 \mathrm{GeV} / c\right)$, requiring the ratio of hadronic to electromagnetic energy in the calorimeter towers to be less than $0.055+0.00045 \cdot E_{E M}$, and various other cuts. Isolated electrons from $W$ decay are preferred over electrons from $b$ or $c$ quark semi-leptonic decay by a cut that requires the total calorimeter energy in a

cone of $\Delta R\left(=\sqrt{\Delta \phi^{2}+\Delta \eta_{\text {evt }}^{2}}\right)=0.4$ around the cluster to be less than $10 \%$ of the cluster energy. Photon conversions to $e^{+} e^{-}$pairs are explicitly reconstructed and rejected.

Muons are identified by a high-momentum track in the tracking detectors $\left(p_{T}>\right.$ $20 \mathrm{GeV} / c$ ) matched with a set of hits in the muon chambers. The calorimeter towers to which the track points must contain energy consistent with a minimum ionizing particle. An isolation cut is imposed, requiring the total calorimeter energy in a cone of $\Delta R=0.4$ around the muon track (excluding the towers through which the muon passed) to be less than $10 \%$ of the track momentum. Cosmic ray muons are explicitly identified and rejected.

Neutrinos are in some sense the simplest particles because they almost never interact in the detector at all. Thus they are identified by "missing energy" in the event. At a hadron collider, much of the beam energy escapes down the beampipe, so we are not able to balance the momentum of the event in three dimensions. However, the particles escaping down the beampipe carry little momentum transverse to the beam direction, so the momentum in the transverse plane does tend to balance. The resolution of the missing energy (opposite of the vector sum of the transverse energy) is related to the scalar sum of the transverse energy as $\sigma\left(\mathscr{E}_{T}\right) \approx 0.4 \sqrt{\sum E_{T}}$. When the missing energy is significantly different from zero, it is evidence that a neutrino has traversed the detector, carrying off its energy.

\subsection{Event Selection}

A software module (TopEventModule) is used to perform some $t \bar{t}$-specific reconstruction. Detailed lepton ID cuts are implemented in this module, as are corrections to the missing energy to account for muon energy that is not deposited in the calorimeter and for corrections to the jets in the event. Finally, the jet clustering is redone, leaving out energy belonging to any well identified electrons.

To select $t \bar{t}$ events in the lepton + jets channel, we require exactly one well identified lepton in the event. The lepton can be a central electron (denoted CEM), a muon 
observed in the CMU and CMP detectors (denoted CMUP), or a muon observed in the CMX detector (denoted CMX). We require the corrected $\mathscr{E}_{T}>20 \mathrm{GeV}$.

In addition to the standard selection, we apply some cuts specific to the top mass analysis. Since we attempt to reconstruct the top and anti-top decay chains, there must be at least four jets in the event (two $b$ jets and two jets from the hadronic $W$ decay). Additional jets are permitted. We consider jet $E_{T}$ after corrections that smooth out detector response variations with $\eta$ and bring data and Monte Carlo particle energy scales into agreement. (These are the level 4 corrections of section 5.1.2.) All jets must have $|\eta|<2.0$. Events in which at least four jets satisfy a tight requirement of $E_{T}>15 \mathrm{GeV}$ will be referred to as "4-jet events." Due to the high purity of tagged events, this mass analysis with $b$ tags requires just three such tight jets and relaxes the cut on the fourth jet to $E_{T}>8 \mathrm{GeV}$; events in which $8 \mathrm{GeV}<E_{T}^{j 4}<15 \mathrm{GeV}$ will be called "3.5-jet events." 5 Positive tags from the SecVtx secondary vertex tagger are used to identify at least one $b$ jet in each event, but only jets with $E_{T}>15 \mathrm{GeV}$ are considered by the tagger.

Figure 4.2 (top left) illustrates the effect that various cuts on the fourth jet $E_{T}$ have on the acceptance of signal and background events. As the $E_{T}^{j 4}$ cut increases from $8 \mathrm{GeV}$ to $15 \mathrm{GeV}$, the number of $t \bar{t}$ events decreases by $24 \%$ while the number of $W b \bar{b}$ background events decreases by $56 \%$. Wb $\bar{b}$ is one of the dominant background processes. The mean and RMS values of the reconstructed mass (see chapter 5) for the background sample increase with the $E_{T}^{j 4}$ cut, while those for the signal samples remain quite stable, as expected (see Figure 4.2, bottom). The cut of $8 \mathrm{GeV}$ on the fourth jet $E_{T}$ was chosen to increase the $t \bar{t}$ acceptance without diluting too much the signal relative to background. However, this cut has not been optimized more thoroughly by investigating its effect on the top mass sensitivity. We perform the analysis using the full sample of $W+\geq 3.5$-jet events, but in addition, we crosscheck the result using the cleaner sample of $W+\geq 4$-jet events. Unless otherwise noted, all plots, tables, and text refer to $W+\geq 3.5$-jet events.

The data used in this analysis are required to come from runs between 141544 and 167716 that have good data quality. The CMX muon dataset is limited to runs after 150145 , corresponding to $\sim 153 \mathrm{pb}^{-1}$. In the data, we require that the primary lepton fired the corresponding trigger; details of the trigger paths used are given in Table 4.1. At level

\footnotetext{
${ }^{5}$ The $E_{T}$ cut of $8 \mathrm{GeV}$ on the fourth jet was initially chosen to match the run I analysis, but note that, in contrast to the run I analysis, the $\eta$ cut is not relaxed for the fourth jet. The acceptance increase from a looser fourth-jet $\eta$ cut was not considered worth the additional complication of using jets from the far-forward region, which is less well understood.
} 

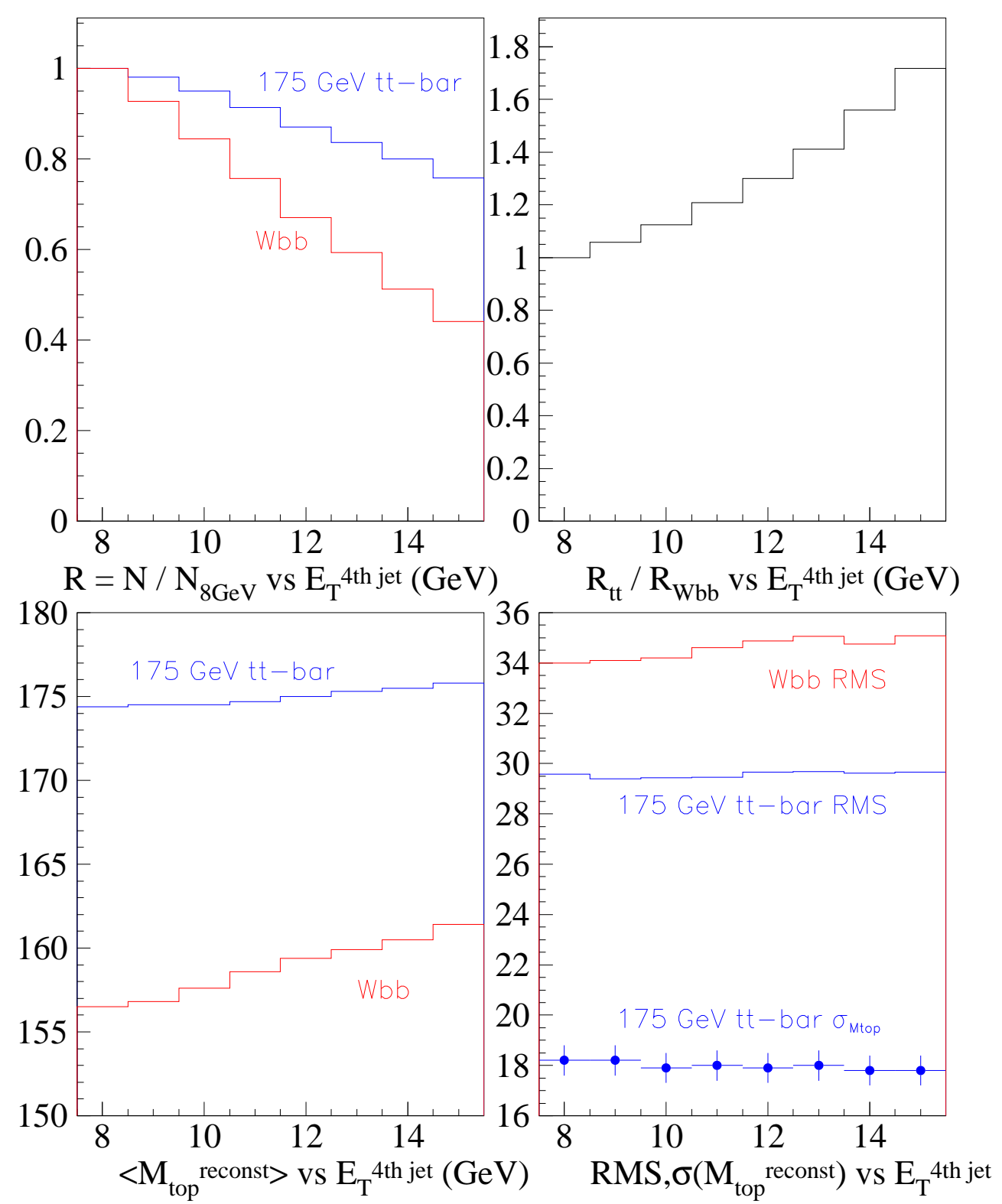

Figure 4.2: Top left: the acceptance as a function of the $E_{T}^{j 4}$ cut with respect to that of the $E_{T}^{j 4}>8 \mathrm{GeV}$ cut: the $t \bar{t}$ sample with $\mathrm{M}_{\mathrm{top}}=175 \mathrm{GeV} / c^{2}\left(R_{t \bar{t}}\right)$ in blue and the $W b \bar{b}$ background sample $\left(R_{W b \bar{b}}\right)$ in red. Top right: $R_{t \bar{t}} / R_{W b \bar{b}}$ as a function of $E_{T}^{j 4}$. Bottom: the average (left) and RMS (right) of the reconstructed top mass distribution between $80 \mathrm{GeV} / c^{2}$ and $380 \mathrm{GeV} / c^{2}$. The width $(\sigma)$ of the Gaussian fit between $140 \mathrm{GeV} / c^{2}$ and $210 \mathrm{GeV} / c^{2}$ is also shown in bottom-right for $t \bar{t}$ (points). 
Table 4.1: The level 1, level 2, and level 3 triggers used to select events for this analysis are shown. A different trigger path is used depending on whether the primary lepton (from $W$ decay) is an electron or a muon. Muons in two different subdetectors also have separate triggers.

\begin{tabular}{|c|c|c|c|}
\hline & CEM Electron & CMUP Muon & CMX Muon \\
\hline Level 1 & L1_CEM8_PT8 & L1_CMUP6_PT4 & L1_CMX6_PT8_CSX \\
& $8 \mathrm{GeV} / c$ XFT track & $4 \mathrm{GeV} / c$ XFT track & $8 \mathrm{GeV} / c$ XFT track \\
& $8 \mathrm{GeV}$ CEM tower & $6 \mathrm{GeV} / c$ CMU stub & $6 \mathrm{GeV} / c$ CMX stub \\
& & CMP stub & \\
\hline Level 2 & L2_CEM16_PT8 & L2_CMUP6_PT8 & L2_CMX6_PT10 \\
& $16 \mathrm{GeV}$ CEM cluster & $8 \mathrm{GeV} / c$ XFT track & $10 \mathrm{GeV} / c$ XFT track \\
\hline Level 3 & ELECTRON_CENTRAL_18 & MUON_CMUP18 & MUON_CMX18 \\
& $18 \mathrm{GeV}$ electron & $18 \mathrm{GeV}$ muon & $18 \mathrm{GeV}$ muon \\
\hline
\end{tabular}

1 , a track is required to be found by the hardware fast tracker XFT, along with a tower with large electromagnetic transverse energy or a stub in the muon chambers. At level 2, more sophisticated electron clustering is performed, and the thresholds are higher. In level 3, a complete reconstruction of the event is performed and a reconstructed electron or muon with simple identification cuts is required. The trigger efficiencies are high, $\sim 96 \%$ for electrons and $\sim 90 \%$ for muons, and show negligible $p_{T}$ dependence for leptons in our sample. We do not expect any distortion of kinematic distributions, such as the reconstructed mass, due to trigger effects. Thus no trigger simulation is run on Monte Carlo events, and no trigger requirement is imposed.

Using the selection described above, a total of 27 4-jet events and 10 3.5-jet events are found with at least one $b$ tag, of which 44 -jet events and 13.5 -jet event have two $b$ tags (see Table 4.2). The selected events are listed in Table 4.3 (events that pass an additional cut in the mass reconstruction, $\left.\chi_{\min }^{2}<9\right)$ and Table 4.4 (events that fail the $\chi_{\min }^{2}<9$ cut). For each event the tables give the run and event numbers, lepton type, lepton $E_{T}$, raw $E_{T}$, the four leading jets' raw $p_{T}$, the reconstructed top mass, and the $\chi_{\text {min }}^{2}$ of the mass reconstruction. A description of the top mass reconstruction, including a definition of $\chi_{\min }^{2}$, is given in chapter 5 . Chapter 9 features various checks on our understanding of the data sample. 
Table 4.2: The number of events found in each jet bin with one or two $b$-tagged jets. The numbers in parentheses indicate the number of events used in the top mass measurement, that is, with $\chi_{\min }^{2}<9$ in mass reconstruction (see chapter 5 for $\chi_{\min }^{2}$ description).

\begin{tabular}{|r||c|c||c|}
\cline { 2 - 4 } \multicolumn{1}{c|}{} & 3.5-jet events & 4-jet events & $\geq 3.5$-jet events \\
\hline Events with $==1$ tag & $9(8)$ & $23(18)$ & $32(26)$ \\
\hline Events with $==2$ tags & $1(1)$ & $4(1)$ & $5(2)$ \\
\hline \hline Events with $\geq 1$ tag & $10(9)$ & $27(19)$ & $37(28)$ \\
\hline
\end{tabular}

Table 4.3: The run and event numbers of the 28 top candidate events used in the top mass measurement, various raw measured variables for each event, and the fitted reconstructed mass and $\chi^{2}$, as described in chapter 5 . These events passed the $\chi_{\min }^{2}<9$ cut in the mass reconstruction. The events marked by " $\nmid$ " have two $b$-tagged jets among the four leading jets.

\begin{tabular}{|c|c|c|c|c|c|c|c|c|}
\hline \multicolumn{9}{|c|}{$\chi_{\min }^{2}<9$ (events used in mass extraction) } \\
\hline Run & Event & $\mathrm{Njt}$ & Type & $\begin{array}{l}E_{T}^{\text {lep }} \\
\mathrm{GeV}\end{array}$ & $\begin{array}{c}E_{T} \\
\mathrm{GeV}\end{array}$ & $\begin{array}{c}p_{T}, \text { four leading jets } \\
\mathrm{GeV} / c\end{array}$ & $\chi^{2}$ & $\begin{array}{c}\mathrm{M}_{\mathrm{top}} \\
\mathrm{GeV} / c^{2}\end{array}$ \\
\hline 141597 & 1353293 & 3.5 & CMUP & 51.2 & 30.9 & $50.3,39.4,36.4,12.0$ & 3.4 & 135.2 \\
\hline 144674 & 1782954 & 3.5 & CEM & 98.7 & 31.6 & $45.4,39.5,36.0,10.5$ & 4.9 & 132.6 \\
\hline 145036 & 245760 & 4 & CMUP & 28.2 & 67.4 & $95.9,64.1,19.1,17.4$ & 5.3 & 175.9 \\
\hline 148153 & 6088 & 3.5 & CEM & 54.9 & 48.2 & $67.0,46.7,18.0,8.8$ & 0.3 & 144.3 \\
\hline 149387 & 2551061 & 3.5 & CEM & 66.8 & 29.7 & $34.0,22.2,17.7,7.9$ & 4.2 & 122.0 \\
\hline 152266 & 3554 & 4 & CMUP & 30.6 & 40.2 & $54.9,40.3,17.4,16.7$ & 0.6 & 153.6 \\
\hline 153693 & 799494 & 4 & CMUP & 54.4 & 37.1 & $94.8,65.7,52.9,36.8$ & 0.4 & 176.8 \\
\hline 153694 & 1694029 & 4 & CEM & 31.8 & 31.5 & $118.8,59.9,36.5,23.3$ & 5.0 & 154.0 \\
\hline 153738 & 2083102 & 4 & CEM & 23.0 & 67.2 & $100.1,48.6,52.5,23.0$ & 2.6 & 216.8 \\
\hline 154175 & 1630925 & 4 & CEM & 30.4 & 115.1 & $111.5,29.4,17.0,15.4$ & 0.8 & 131.6 \\
\hline$\dagger 155145$ & 132579 & 3.5 & CEM & 52.9 & 31.7 & $65.7,59.8,27.2,14.2$ & 0.3 & 169.9 \\
\hline 155345 & 3194866 & 3.5 & CEM & 26.4 & 61.9 & $41.6,40.2,13.6,7.8$ & 3.8 & 122.9 \\
\hline 160153 & 1270879 & 4 & CEM & 93.2 & 52.1 & $87.6,50.2,24.0,16.3$ & 0.2 & 170.2 \\
\hline 160437 & 280173 & 4 & CMUP & 43.1 & 29.9 & $56.5,51.3,49.2,17.2$ & 0.1 & 178.6 \\
\hline 161379 & 494836 & 4 & CEM & 155.4 & 29.7 & $94.0,37.8,30.1,15.2$ & 1.3 & 152.8 \\
\hline 161633 & 1571961 & 4 & CEM & 48.6 & 36.8 & $75.3,46.2,24.3,13.1$ & 2.6 & 134.5 \\
\hline 161788 & 361577 & 4 & CMUP & 37.1 & 62.9 & $123.7,87.5,60.4,53.1$ & 0.7 & 177.1 \\
\hline 162423 & 261933 & 4 & CEM & 73.3 & 30.5 & $36.9,27.9,26.0,15.1$ & 0.9 & 129.6 \\
\hline 162519 & 931923 & 3.5 & CMX & 72.6 & 33.8 & $36.9,24.2,16.6,11.9$ & 3.3 & 131.1 \\
\hline 162631 & 7109631 & 4 & CMX & 84.5 & 22.3 & $70.7,34.4,18.4,12.0$ & 4.5 & 169.8 \\
\hline 162986 & 1538897 & 4 & CEM & 52.2 & 51.7 & $102.2,59.1,38.3,25.4$ & 1.5 & 264.6 \\
\hline 163012 & 2249546 & 4 & CMUP & 80.2 & 34.5 & $93.4,57.2,29.4,24.3$ & 6.4 & 192.3 \\
\hline 164274 & 2932602 & 3.5 & CMUP & 77.6 & 24.2 & $30.0,29.7,19.6,8.4$ & 1.3 & 148.6 \\
\hline 164819 & 2297394 & 4 & CEM & 31.5 & 46.8 & $33.1,35.4,15.9,15.4$ & 0.7 & 159.2 \\
\hline 165314 & 1155563 & 3.5 & CMUP & 28.8 & 12.6 & $44.4,20.8,18.6,8.3$ & 1.0 & 126.4 \\
\hline 166007 & 498553 & 4 & CEM & 44.7 & 52.7 & $65.2,54.9,43.1,13.9$ & 2.9 & 190.5 \\
\hline$\dagger 166567$ & 11615607 & 4 & CMUP & 39.7 & 50.1 & $69.0,75.4,36.0,31.0$ & 5.6 & 247.0 \\
\hline 166614 & 804529 & 4 & CEM & 111.2 & 29.4 & $97.3,51.1,42.1,20.1$ & 0.6 & 184.6 \\
\hline
\end{tabular}


Table 4.4: The run and event number of the nine top candidate events that failed the $\chi_{\min }^{2}<9$ cut in the mass reconstruction, various raw measured variables for each event, and the fitted reconstructed mass and $\chi^{2}$, as described in chapter 5. These events passed the $\chi_{\min }^{2}<9$ cut in the mass reconstruction. The events marked by " $\dagger$ " have two $b$-tagged jets among the four leading jets.

\begin{tabular}{|r|r|c|c|c|c|c|c|c|}
\hline \multicolumn{10}{|c|}{$\chi_{\min }^{2}>9$} \\
\hline Run & Event & Njt & Type & $\begin{array}{c}E_{T}^{\text {lep }} \\
\mathrm{GeV}\end{array}$ & $\begin{array}{c}E_{T} \\
\mathrm{GeV}\end{array}$ & $\begin{array}{c}p_{T}, \text { four leading jets } \\
\mathrm{GeV} / c\end{array}$ & $\chi^{2}$ & $\begin{array}{c}\mathrm{M}_{\text {top }} \\
\mathrm{GeV} / c^{2}\end{array}$ \\
\hline 155320 & 480816 & 4 & CEM & 99.4 & 28.1 & $51.0,31.3,19.3,16.4$ & 41.6 & 146.2 \\
$\dagger 156116$ & 6116596 & 4 & CEM & 25.3 & 28.2 & $69.8,51.2,29.4,27.9$ & 367 & 231.7 \\
160591 & 894406 & 4 & CMUP & 114.8 & 110.5 & $148.8,67.5,55.6,14.4$ & 9.6 & 232.2 \\
161013 & 111162 & 4 & CEM & 78.6 & 71.9 & $96.6,90.0,21.3,20.8$ & 9.6 & 172.6 \\
161678 & 5912849 & 4 & CEM & 27.6 & 54.7 & $97.4,75.4,38.9,28.0$ & 10.3 & 200.0 \\
165313 & 1770456 & 3.5 & CMUP & 112.0 & 22.9 & $85.8,18.7,12.9,12.7$ & 20.9 & 137.8 \\
166805 & 2534588 & 4 & CMUP & 59.1 & 56.8 & $123.9,27.3,27.1,17.6$ & 13.6 & 172.1 \\
$\dagger 167139$ & 1191211 & 4 & CMX & 31.2 & 63.5 & $56.6,38.7,37.3,21.5$ & 13.6 & 175.0 \\
$\dagger 167551$ & 7969376 & 4 & CEM & 77.2 & 54.9 & $75.8,38.5,24.9,23.1$ & 174 & 278.0 \\
\hline
\end{tabular}

\subsection{Expected Backgrounds}

The source and number of background events in the tagged sample were studied for the $W+3.5$-jet bin and the $W+\geq 4$-jet bin. Table 4.5 summarizes the background estimates for the $W+3.5$-jet sample and the $W+\geq 4$-jet sample, before the $\chi^{2}$ cut in the mass reconstruction. The dominant contributions (totaling about $75 \%$ ) come from $W+$ jets (mistags), non- $W$ (QCD), and $W b \bar{b}$ events. The uncertainty on the total number of background events is calculated by assuming $100 \%$ correlation for uncertainties in different jet bins of the same process, and no correlation for uncertainties on different processes, except for the $W+$ heavy flavor backgrounds, which are correlated through the uncertainty on the heavy flavor fraction. The efficiency of the $\chi^{2}$ cut on background events will be discussed in section 6.2 . 
Table 4.5: The sources and expected numbers of background events in the $W+3.5$-jet sample and the $W+\geq 4$-jet sample, for $162 \mathrm{pb}^{-1}$.

\begin{tabular}{|c||c|c|}
\hline \multicolumn{1}{|c||}{ Source } & \multicolumn{2}{c|}{ Expected Background } \\
\cline { 2 - 3 } & $W+3.5$ jets & $W+\geq 4$ jets \\
\hline$W+$ jets (mistags) & $1.6 \pm 0.3$ & $1.2 \pm 0.3$ \\
$W b \bar{b}$ & $1.0 \pm 0.4$ & $0.6 \pm 0.3$ \\
$W c \bar{c}$ & $0.4 \pm 0.2$ & $0.2 \pm 0.1$ \\
$W c$ & $0.5 \pm 0.2$ & $0.2 \pm 0.1$ \\
$W W / W Z$ & $0.17 \pm 0.03$ & $0.12 \pm 0.02$ \\
non- $W$ (QCD) & $1.7 \pm 0.5$ & $1.7 \pm 0.5$ \\
Single top (S chan.) & $0.18 \pm 0.03$ & $0.13 \pm 0.02$ \\
Single top (T chan.) & $0.12 \pm 0.02$ & $0.06 \pm 0.02$ \\
\hline Total & $5.7 \pm 0.8$ & $4.4 \pm 0.7$ \\
\hline
\end{tabular}




\section{Chapter 5}

\section{Top Mass Reconstruction}

For each event that has been selected according to chapter 4, we extract a single quantity through a series of corrections to the raw detector measurements and a multiparameter fit in MINUIT [20]. ${ }^{1}$ This quantity is intended to have a strong dependence on the top quark pole mass, so that we can use its distribution in the data to determine the top mass through a likelihood fit to the distributions we extract from various Monte Carlo samples (see chapter 6).

Specifically, we do our best to reconstruct the invariant mass of the top quark in each event, assuming the event is $t \bar{t}$ signal with top quark decays and kinematics according to standard model predictions. Every event is treated identically, whether signal or background, data or Monte Carlo (except as noted below). Some events "fail" the mass reconstruction and are discarded. This amounts to one more event selection requirement, and will be described below.

Note that, although this quantity is referred to as the reconstructed top mass, it is not used as an independent measurement of the top mass in each event. It is simply an experimental quantity that will be used to discriminate among the range of possible true values of the standard model top quark mass.

\subsection{Correcting Measured Quantities}

We provide as input to the MINUIT fit the four-vectors of the various physics objects identified in the event. We first apply all known corrections to the measured quan-

\footnotetext{
${ }^{1}$ MINUIT is a standard minimization package used by particle physicists since time immemorial.
} 
tities, and establish an uncertainty on the quantities that are permitted to vary in the fit. These corrections are performed by TopEventModule (see section 4.3) and a software module called TopMassFitModule, which also performs the fit described in this chapter.

\subsubsection{Lepton corrections}

The electron four-vector is defined as follows. The electron energy is taken from a two-tower calorimeter cluster, while the angles are taken from the beam-constrained COTonly track. We apply corrections to the energy for slight differences in the response of the electromagnetic calorimeter depending on where in the tower face the electron enters. The electron mass is set to zero in the four-vector, and the angles are taken as perfectly measured quantities. The transverse momentum of the electron is given an uncertainty of

$$
\frac{\sigma_{p_{T}^{e}}}{p_{T}^{e}}=\sqrt{\left(\frac{0.135}{\sqrt{p_{T}^{e}[\mathrm{GeV} / c]}}\right)^{2}+(0.02)^{2}}
$$

Since the angles are fixed, this amounts to an uncertainty on the magnitude of the full electron momentum vector, not just the transverse component.

For muons, the corrected four-vector uses the three-vector of the beam-constrained COT-only track, also with a mass of zero. We apply in addition muon curvature corrections due to chamber misalignment:

$$
\frac{1}{p_{T}^{\mu}[\mathrm{GeV} / c]}=\frac{1}{p_{T}^{\mu}[\mathrm{GeV} / c]}-0.00037-0.00110 \sin (\phi+0.28)
$$

(data only; no such correction is applied to Monte Carlo muons). Again the angle and mass are given no uncertainty; the transverse momentum is given an uncertainty of

$$
\sigma_{1 / p_{T}^{\mu}[\mathrm{GeV} / c]}=0.0011 \quad \rightarrow \quad \frac{\sigma_{p_{T}^{\mu}}}{p_{T}^{\mu}}=0.0011 \cdot p_{T}^{\mu}[\mathrm{GeV} / c]
$$

which is equivalent to an uncertainty on $|p|$, as for electrons. The electron and muon transverse momentum uncertainties are taken from studies performed in run I. (The relevant detectors are unchanged.)

\subsubsection{Generic jet corrections}

Jets are first corrected with a set of "generic" jet corrections, so called because they are intended to be independent of the particular process under consideration. The 
generic jet corrections consist of seven "levels" applied in series, each of which scales the jet four-vector to account for a particular effect, as follows.

Level 1: $\eta$-Dependent Calibration The $\eta$ dependence in calorimeter response with respect to the central calorimeter is removed by applying corrections derived from the dijet balancing procedure. This procedure assumes that the magnitudes of transverse momenta of the jets in two-jet events be equal on average. One of the two jets is required to be in the central region with $0.2<|\eta|<0.6$, and $\eta$-dependent corrections are derived such that the $p_{T}$ of the other jet balances on average the $p_{T}$ of the central jet. A different dependence on $\eta$ is observed in simulated events and data events, so the extracted relative corrections are different. After this correction, all jets are normalized to central jets, and all subsequent corrections are determined for jets with $0.2 \leq|\eta| \leq 0.6$.

Level 2: Unused This correction for time-dependent gain in the PMTs is now performed at the level of tower calibrations.

Level 3: Raw Energy Scale This simple scale factor returns the energy scale in the central calorimeter to that measured in the test beam for a single particle. This correction is also different for simulated events and real data. After this point, Monte Carlo jets and data jets are equivalent, and subsequent corrections do not distinguish between the two.

Level 4: Multiple Interactions When more than one $p \bar{p}$ pair interact in a given bunch crossing, the debris from additional collisions can fall into the jet cone, increasing the energy. The average energy deposited in a tower as a function of the number of reconstructed primary vertices is determined from minimum bias data, and a correction is applied to reverse the effect.

Level 5: Absolute Energy Correction The CDF calorimeters are non-linear devices; they respond differently to particles with different energies. At this level, jets are corrected for the effects of fragmentation and calorimeter non-linearity. The corrections are derived from Monte Carlo simulation, and after this point, the jet energy should on average represent the total energy of the hadrons inside the jet cone.

Level 6: Underlying Event For a given $p \bar{p}$ collision, part of the original proton and 
anti-proton momentum not involved in the hard parton interaction can flow into the calorimeter, artificially inflating the jet energies. The correction is derived from minimum bias events where there is exactly one reconstructed primary vertex.

Level 7: Out-of-Cone Correction When the goal is to determine the original parton energy from a measurement of the jet energy, it must be taken into account that a parton's total energy may not remain inside the cone of jet clustering, especially for the relatively small cone size of 0.4 used in this analysis. This correction, derived from simulated events, adds back in the energy that, on average, falls outside of the jet cone.

Note that the corrections at levels 5 and 7 , since they are a function of $p_{T}^{j e t}$ and since jet resolution is non-negligible, necessarily incorporate information about an underlying $p_{T}$ distribution. In particular, for these generic jet corrections, a flat jet $p_{T}$ spectrum is assumed.

For the purposes of mass fitting (as opposed to event selection), we apply generic jet corrections through level 5. To summarize, these corrections are intended to return, on average, the true in-cone hadronic energy for a jet in a dijet event with flat $p_{T}$ spectrum. That is, they are intended to correct for differences in the calorimeter response at different $\eta$, an overall energy scale calibration, extra energy derived from multiple collisions in the same accelerator bunch crossing, and the calorimeter non-linearity. Generic jet corrections simply scale a jet four-vector by a factor, so angles are unaffected, while $E$ and $p$ (and mass!) change. The various sources of uncertainty in the CDF jet energy scale and simulation are quantified in the systematic uncertainties on these jet corrections (see section 8.1).

\subsubsection{Top-specific jet corrections}

Jet corrections derived specifically for the $t \bar{t}$ process are needed to account for the different fragmentation of light quark jets from $W$-boson decay ( $W$ jets) and $b$ jets, as well as the non-flat $p_{T}$ spectrum of partons from $t \bar{t}$ decay. In addition, the top-specific corrections account for the energy falling outside the jet cone. ${ }^{2} W$ jets and $b$ jets, which

\footnotetext{
${ }^{2}$ The level 7 corrections are not applicable for $t \bar{t}$ events, since they are determined using dijet events, where there is no jet overlap. In the case of busy $t \bar{t}$ events, naively applying the generic out-of-cone corrections results in the double-counting of some energy.
} 
clearly have different response (see Figure 5.1), are corrected using different functions, but no separate correction is attempted for $b$ jets with identified semi-leptonic decays.

The $t \bar{t}$-specific corrections also provide uncertainties on the jet energies (i.e. the measurement resolution, not systematic uncertainties). As in the case of the lepton fourvectors, the angles of jets are assumed to be perfectly measured; only the $\sigma_{p_{T}^{j e t}}$ is parameterized.

The $t \bar{t}$-specific corrections are extracted from a large sample of HERWIG $t \bar{t}$ events in which the four leading jets in $E_{T}$ are matched with the four HEPG partons from $t \bar{t}$ decay. ${ }^{3}$ The correction is defined as (the inverse of) the most probable value of the jet response curve, as a function of $p_{T}^{\text {jet }}$ and $\eta^{j e t}$. A corresponding resolution is found by taking the symmetric window about the MPV of the jet response that includes $68 \%$ of the total area.

Figure 5.1 illustrates the need for the $t \bar{t}$-specific corrections. The upper plot shows significant differences between parton momentum and jet momentum with $p_{T}^{\text {jet }}<40 \mathrm{GeV} / c$ after generic corrections. The $t \bar{t}$-specific corrections remove the dependence of the jet response as a function of $p_{T}^{\text {jet }}$ separately for $b$-jets and $W$-jets. In addition, the $p_{T}$ spectrum of jets from $t \bar{t}$ decay is strongly dependent on $\eta$. Therefore we expect $\eta$ dependence in the jet response after any $t \bar{t}$-specific corrections that depend only on $p_{T}^{j e t}$. This is illustrated in the lower plot of Figure 5.1. The corrections are extracted as a function of both $p_{T}^{j e t}$ and $\eta^{j e t}$ in order to remove this effect.

One way to check the effectiveness of the $t \bar{t}$-specific corrections is to look at the reconstructed invariant mass of the jets matched to the $W$-boson daughters as a function of the $p_{T}^{W}$ and average $\eta$ of daughter jets. As shown in Figure 5.2, no significant dependences are observed after applying the $t \bar{t}$-specific corrections. The overall $W$ mass distribution has mean and Gaussian mean of $78.6 \mathrm{GeV} / c^{2}$ and $79.5 \mathrm{GeV} / c^{2}$, respectively. We also look at the effect on the reconstructed invariant mass of the three jets from the hadronic top. The $t \bar{t}$-specific corrections have been shown to improve the RMS of the hadronic invariant mass distribution by $\approx 10 \%$.

The $t \bar{t}$-specific corrections produce another scale factor by which the full jet fourvector is multiplied. Thus again the angles do not change. Note that, since these corrections depend on the flavor of the jet, they must be applied after a hypothesis has been selected

\footnotetext{
${ }^{3}$ As a crosscheck, the correction functions and their effect on the reconstructed invariant mass of the hadronic top quark have been shown to be consistent with those extracted from a large Pythia sample.
} 

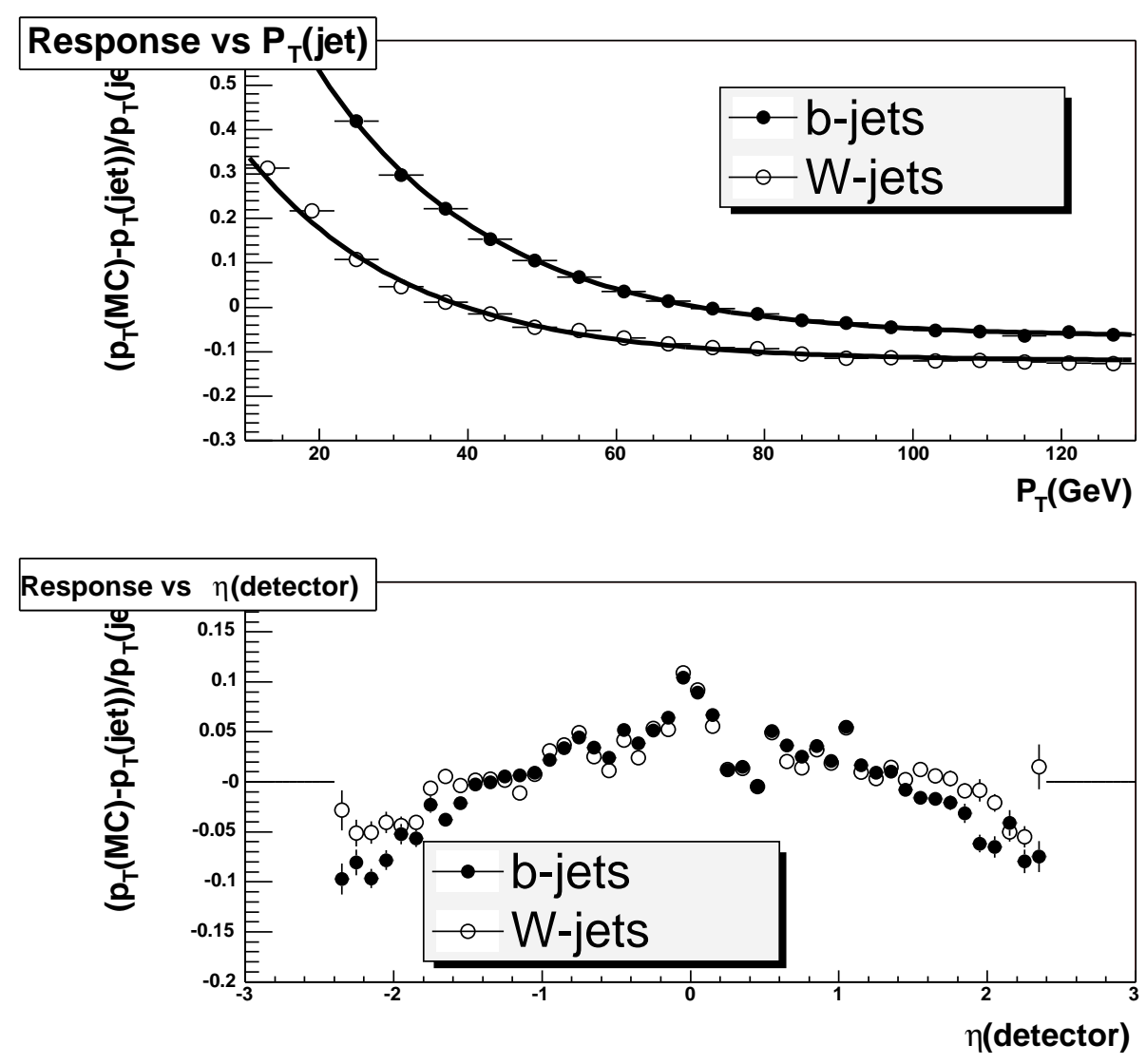

Figure 5.1: Upper plot: jet response, $\left(p_{T}^{\text {parton }}-p_{T}^{\text {jet }}\right) / p_{T}^{\text {jet }}$, as a function of $p_{T}^{\text {jet }}$ for $b$-jets and $W$-jets after only generic corrections are applied. Lower plot: jet response as a function of $\eta^{j e t}$ after applying $t \bar{t}$-specific corrections that depend only on $p_{T}^{\text {jet }}$. 

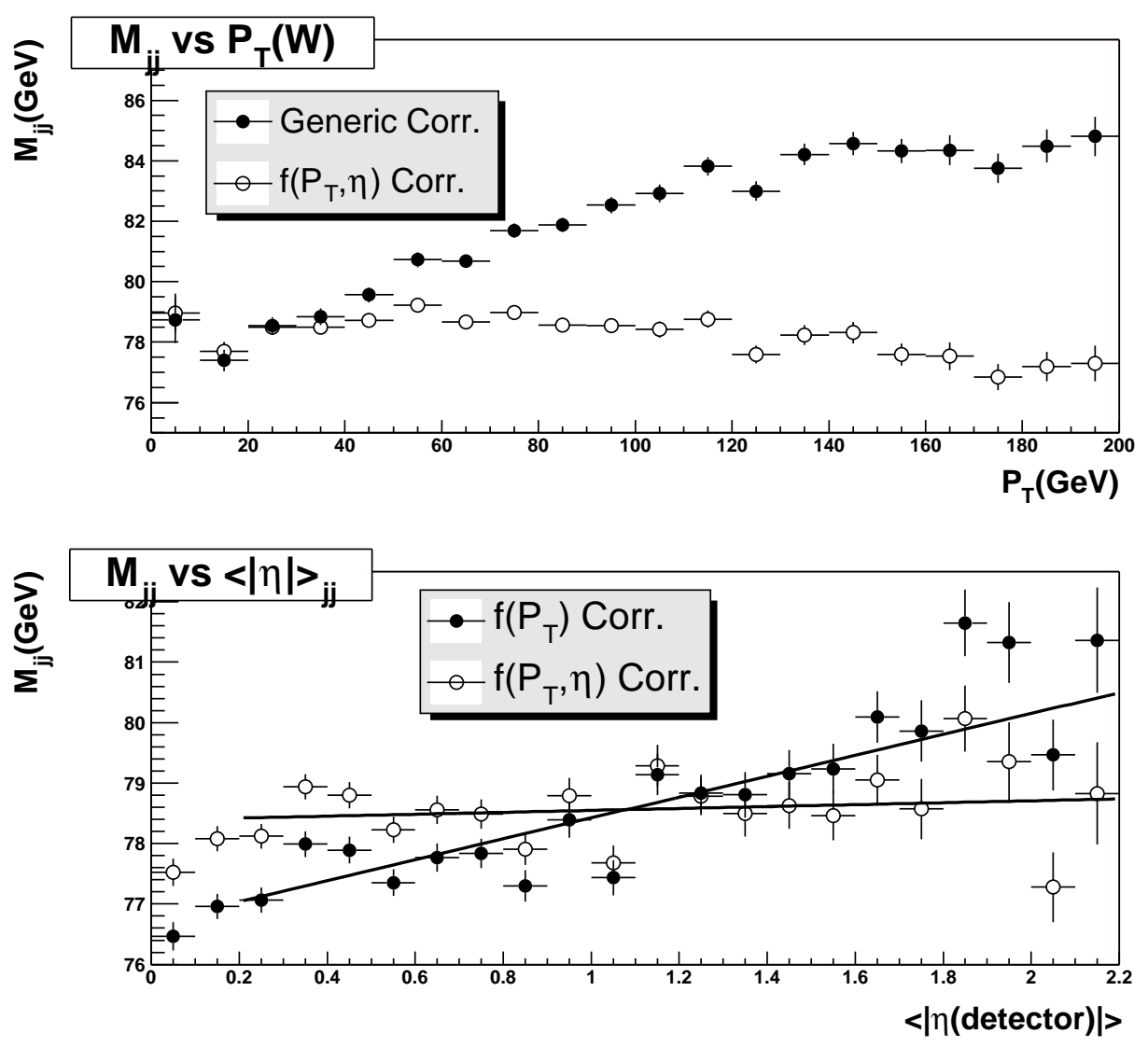

Figure 5.2: Upper plot: invariant mass of jets from $W$ daughters as a function of $p_{T}^{W}$ after applying generic corrections (full circles) and $p_{T^{-}}$and $\eta$-dependent corrections (open circles). Lower plot: invariant mass of jets from $W$ daughters as a function of the average $\eta$ of $W$ jets after applying only $p_{T^{-}}$-dependent corrections (full circles) and $p_{T^{-}}$and $\eta$-dependent corrections (open circles). In both plots, MC events with a good jet-parton match are used. 
for the assignment of the measured jets to partons from the $t \bar{t}$ decay chain.

As a final step, the jet momentum is held fixed while the jet energy is adjusted so that the jet has a mass according to its flavor hypothesis. A mass of $0.5 \mathrm{GeV}$ is used for $W$ jets, and a mass of $5.0 \mathrm{GeV}$ is used for $b$ jets.

\subsubsection{Additional jets, unclustered energy, and missing energy}

"Additional jets" in an event are those jets beyond the leading four in $E_{T}$ corrected through level 4 , but still having $E_{T}>8 \mathrm{GeV}$ and $|\eta|<2.0$. They are identified as not belonging to the top decay chain, but arising from initial state radiation, underlying event, or multiple interactions. This is, of course, an approximation, since the additional jets may in fact be top decay daughters, or may arise from final state radiation and therefore be relevant in reconstructing the top quark mass. These jets are corrected using generic corrections through level 5; however, they contribute to the reconstructed top mass only through their effect on the $\mathscr{E}_{T}$ calculation.

The "unclustered energy" in an event consists of any energy seen in the calorimeter that is not associated with the primary lepton or a jet. The $p_{T}$ of towers with $|\eta|<3.6$ are included in the unclustered energy. ${ }^{4}$ The "missing energy" is identified with the energy carried out of the detector by one or more neutrinos. The unclustered energy and missing energy are related to each other through the other measured physics objects in the event, since the $p \bar{p}$ system has $p_{T}=0$.

In this analysis, the input to the MINUIT fit called unclustered energy is the sum of the raw unclustered energy $U_{T}^{r a w}$, described above, and the energies of the additional jets. The raw unclustered energy is multiplied by a scale factor of 1.4, which is roughly the level 5 correction factor for $8 \mathrm{GeV}$ jets. In addition, we add to the unclustered energy the energy that would have been subtracted from the leading four jets due to the underlying energy (level 6 generic corrections), and we subtract the energy that would have been added to those jets due to energy falling outside the jet cone (level 7 generic corrections). This is done to avoid double-counting of energy that is included in the jet energies after all corrections. So the unclustered energy input to the fit is the vector sum

$$
\overrightarrow{U_{T}}=1.4 * \overrightarrow{U_{T}^{r a w}}+\sum_{\text {add'l jets }} E_{T}^{\overrightarrow{j e t}}+\sum_{t \bar{t} \text { jets }} E_{T}^{\vec{L} 6}-\sum_{t \bar{t} \text { jets }} E_{T}^{\vec{L} 7}
$$

\footnotetext{
${ }^{4}$ Jets with $E_{T}>8 \mathrm{GeV}$ but $|\eta|>2.0$ are not considered as separate physics objects in this analysis, so their raw energy contributes to the unclustered energy like any other tower.
} 
Each transverse component of this composite unclustered energy $\left(U_{x}, U_{y}\right)$ is assigned an uncertainty of $0.4 \cdot \sqrt{\sum E_{T}^{\text {unclus }}}$, where $\sum E_{T}^{\text {unclus }}$ is the scalar sum of the transverse energy of towers not included in the electron or one of the leading four jet clusters (cf. section 3.3.2).

The neutrino energy, i.e. the missing energy, is always calculated from the other objects, including the composite unclustered energy. In practice, only the transverse momentum in the event balances, so only the neutrino transverse momentum $p_{T}^{\nu}$ is calculated at each stage in the fit, using the current values of lepton, jet, and unclustered transverse energies:

$$
\overrightarrow{p_{T}}=-\left(\overrightarrow{p_{T}^{\ell}}+\sum p_{T}^{\overrightarrow{j e t}}+\overrightarrow{U_{T}}\right)
$$

Note that this quantity, used in the mass fitting procedure, is different from the missing energy described in section 4.2 and used in event selection.

Although this is a complicated piece of the fit procedure, and it is not clear what exactly is the "correct" way to treat unclustered energy and the missing energy, we have concluded that the $E_{T}$ calculation does not in fact have a large effect on the results of the $\chi^{2}$ fit. Various other approaches to correcting the unclustered energy and assigning resolution were tried, and no changes had any significant effect on the top mass resolution.

The longitudinal momentum of the neutrino $p_{z}^{\nu}$ is a free (unconstrained) parameter in the fit. Its initial value is calculated using the initial value of the lepton four-vector and the initial missing energy, and assuming their mother $W$ has its pole mass. Since these conditions yield a quadratic equation, there are in general two solutions for the $p_{z}^{\nu}$; a separate fit is done with each solution used as the initial value of $p_{z}^{\nu}$. When the two fits land in different minima, we choose the one that provides the best fit using the $\chi^{2}$ values as described below.

\subsection{MINUIT fit}

Given the inputs described above, the event-by-event fit for the reconstructed top mass proceeds as follows. MINUIT is used to minimize a $\chi^{2}$ where the top mass is a free parameter. For each event this is done 24 times, corresponding to the 12 ways to assign the four leading jets to the four partons in $t \bar{t}$ decay, ${ }^{5}$ times the 2 solutions for the neutrino

\footnotetext{
${ }^{5}$ Although $4 \cdot 3 \cdot 2 \cdot 1=24$, there is a two-fold degeneracy in the jet-parton assignments since the $W$ daughters are treated identically in the fit.
} 
longitudinal momentum. For each combination, the following $\chi^{2}$ is minimized:

$$
\begin{aligned}
\chi^{2} & =\Sigma_{i=\ell, 4 j e t s} \frac{\left(p_{T}^{i, f i t}-p_{T}^{i, \text { meas }}\right)^{2}}{\sigma_{i}^{2}}+\Sigma_{j=x, y} \frac{\left(U_{j}^{\text {fit }}-U_{j}^{\text {meas }}\right)^{2}}{\sigma_{j}^{2}} \\
& +\frac{\left(M_{j j}-M_{W}\right)^{2}}{\Gamma_{W}^{2}}+\frac{\left(M_{\ell \nu}-M_{W}\right)^{2}}{\Gamma_{W}^{2}}+\frac{\left(M_{b j j}-M_{t}\right)^{2}}{\Gamma_{t}^{2}}+\frac{\left(M_{b \ell \nu}-M_{t}\right)^{2}}{\Gamma_{t}^{2}} .
\end{aligned}
$$

In the first term, $\sigma_{\ell}$ and $\sigma_{j e t}$ are the lepton and four leading jet $p_{T}$ resolutions. In the second term, $p_{x, y}^{U E}$ and $\sigma_{x, y}$ are the components of the composite unclustered energy and their resolutions. These quantities have all been defined in section 5.1.

Each of $M_{j j}, M_{\ell \nu}, M_{b j j}$, and $M_{b \ell \nu}$ refers to the invariant mass of the sum of the four-vectors denoted in the subscript. For example, $M_{j j}$ is the invariant mass of the vector sum of the $W$ daughter jets. $M_{W}$ and $M_{t}$ are the pole masses of the $W$ and top quark. $M_{W}$ is $80.41 \mathrm{GeV} / c^{2}$, taken from the PDG, and $M_{t}$ is, of course, the free parameter that we will take as the reconstructed mass after the $\chi^{2}$ is minimized. The fit is initialized with $M_{t}=175 \mathrm{GeV} / c^{2} . \Gamma_{W}$ and $\Gamma_{t}$ are the total width of the $W$ boson and the top quark. ${ }^{6} \Gamma_{W}$ is $2.06 \mathrm{GeV}$, again from the PDG, and $\Gamma_{t}$ is $2.5 \mathrm{GeV} .^{7}$ Thus these terms provide constraints such that the $W$ masses come out correctly, and the $t$ and $\bar{t}$ masses come out the same (modulo the Breit-Wigner a.k.a. Gaussian distribution in both cases).

The jet-parton assignment (and $p_{z}^{\nu}$ solution) with the lowest $\chi^{2}$ after minimization is selected for each event. The $\chi^{2}$ of this combination is denoted $\chi_{\min }^{2}$, and the requirement $\chi_{\min }^{2}<9$ is imposed. ${ }^{8}$ This value of the $\chi^{2}$ cut was found to give the best expected statistical uncertainty on the top mass, as shown in Figure 5.3. For this analysis, $b$-tagging information is taken into account simply by ignoring combinations that assign a tagged jet to a light quark at parton level. So at this stage, after mass reconstruction, each event either has been rejected or can be described by a single value of this reconstructed mass variable. Indeed, the rest of the analysis can be performed with nothing more than a list of the reconstructed masses for events in various samples that have passed all selection cuts (including the $\chi^{2}$ cut).

\footnotetext{
${ }^{6}$ The $W$ and $t$ have lineshapes described by a Breit-Wigner, not by a Gaussian. It is not clear what one should use for $\sigma$ if one wants to approximate a Breit-Wigner by a Gaussian; currently we use the Breit-Wigner full width at half maximum.

${ }^{7}$ For historical reasons, this is larger than the commonly referenced theoretical top width of $\approx 1.5 \mathrm{GeV}$. In fact, either value is small compared to the jet resolution, so the fit is not expected to be sensitive to this difference.

${ }^{8}$ When the context is unambiguous, we may use $\chi^{2}$ and $\chi_{\min }^{2}$ interchangeably to denote the $\chi^{2}$ of the selected combination for each event.
} 


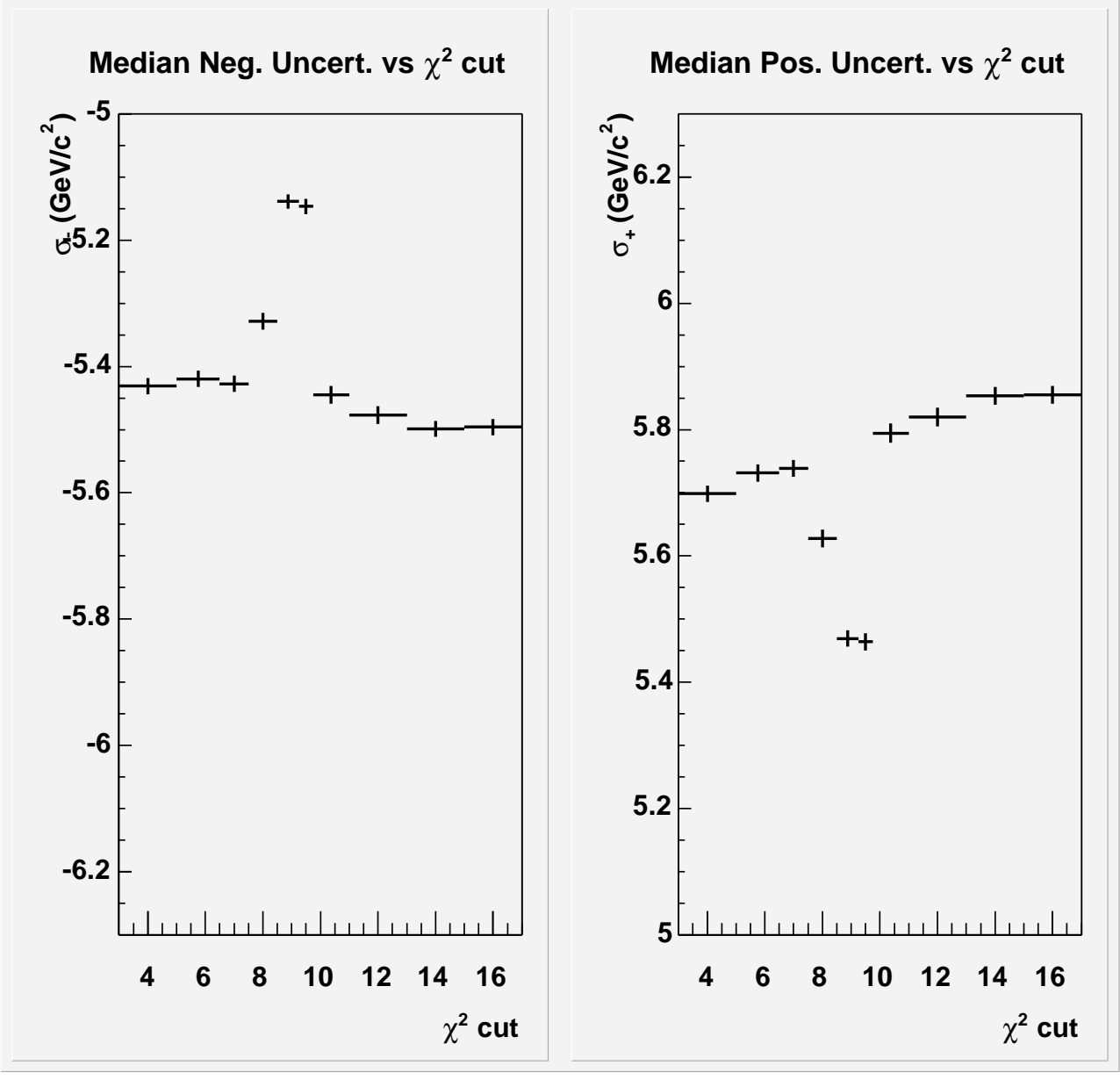

Figure 5.3: The expected statistical uncertainty on the top mass is given as a function of the $\chi^{2}$ cut. The number of events per pseudoexperiment is changed for each value of the $\chi^{2}$ cut, so that the effect of the cut efficiency on the fit statistics is taken into account. 
Figure 5.4 (top) shows the distribution of $\chi_{\min }^{2}$ for a $175 \mathrm{GeV} / c^{2} t \bar{t}$ signal sample, and for $W+$ jets events (mistags), one important background sample. The signal events are more sharply peaked at low values of $\chi^{2}$, but the separation is not overwhelming. The bottom part of Figure 5.4 gives the efficiency of the $\chi^{2}$ cut as a function of the cut value. Our cut at 9 is $\sim 80 \%$ efficient for signal and $\sim 70 \%$ efficient for the background. Also shown are the ratio of signal efficiency to background efficiency $(S / B)$ and the ratio of signal efficiency to the square root of the background efficiency $(S / \sqrt{B})$. The $\chi_{\min }^{2}<9$ cut is also expected to distinguish between signal events where the leading four jets are associated with the top decay daughters, and those where they are not.

A typical reconstructed mass distribution for signal Monte Carlo (this is from the large $175 \mathrm{GeV} / c^{2}$ mass sample) is shown as the yellow histogram in Figure 5.5. Each event in the sample that passes both event selection and the $\chi_{\min }^{2}<9$ cut contributes exactly one entry to this plot. ${ }^{9}$ As expected, the distribution peaks near the generated mass of $175 \mathrm{GeV} / c^{2}$, but we neither seek nor expect an exact correspondence between the generated mass and, say, the mean of the reconstructed mass. The broader shape, beneath the relatively sharp peak at $175 \mathrm{GeV} / c^{2}$, is believed to comprise events where an incorrect assignment of jets to partons has been chosen in the fit. The green histogram in the same figure shows the reconstructed mass distribution for the correct jet-parton assigment (for events where the four leading jets are matched to the four partons from $t \bar{t}$ decay), and this distribution has much smaller tails.

The reconstructed mass distribution for the 28 events passing the $\chi^{2}$ cut in the data can be seen as the yellow histogram in Figure 7.1. These events consist of both $t \bar{t}$ signal and background events. See section 6.2 for a discussion of the expected background shape and normalization.

\footnotetext{
${ }^{9}$ Since the efficiency of the SecVtx $b$ tagger differs in data and Monte Carlo, the ratio of double- to single-tagged events will differ as well. We have checked that scaling the contributions of the two types of events in our signal templates does not affect the result (see section 8.8). If $N_{1}$ is the number of single-tagged events in $\mathrm{MC}, N_{2}$ is the number of double-tagged events in $\mathrm{MC}$, and $x$ is the scale factor $\epsilon_{d a t a} / \epsilon_{M C}$, then the ratio will be correct for data if we use the scaling

$$
\begin{aligned}
& N_{2} \rightarrow N_{2} x^{2} \\
& N_{1} \rightarrow N_{1} x+2 N_{2} x-2 N_{2} x^{2}
\end{aligned}
$$
}

With the current $b$ tagging code, the scale factor is $x=0.89$. 

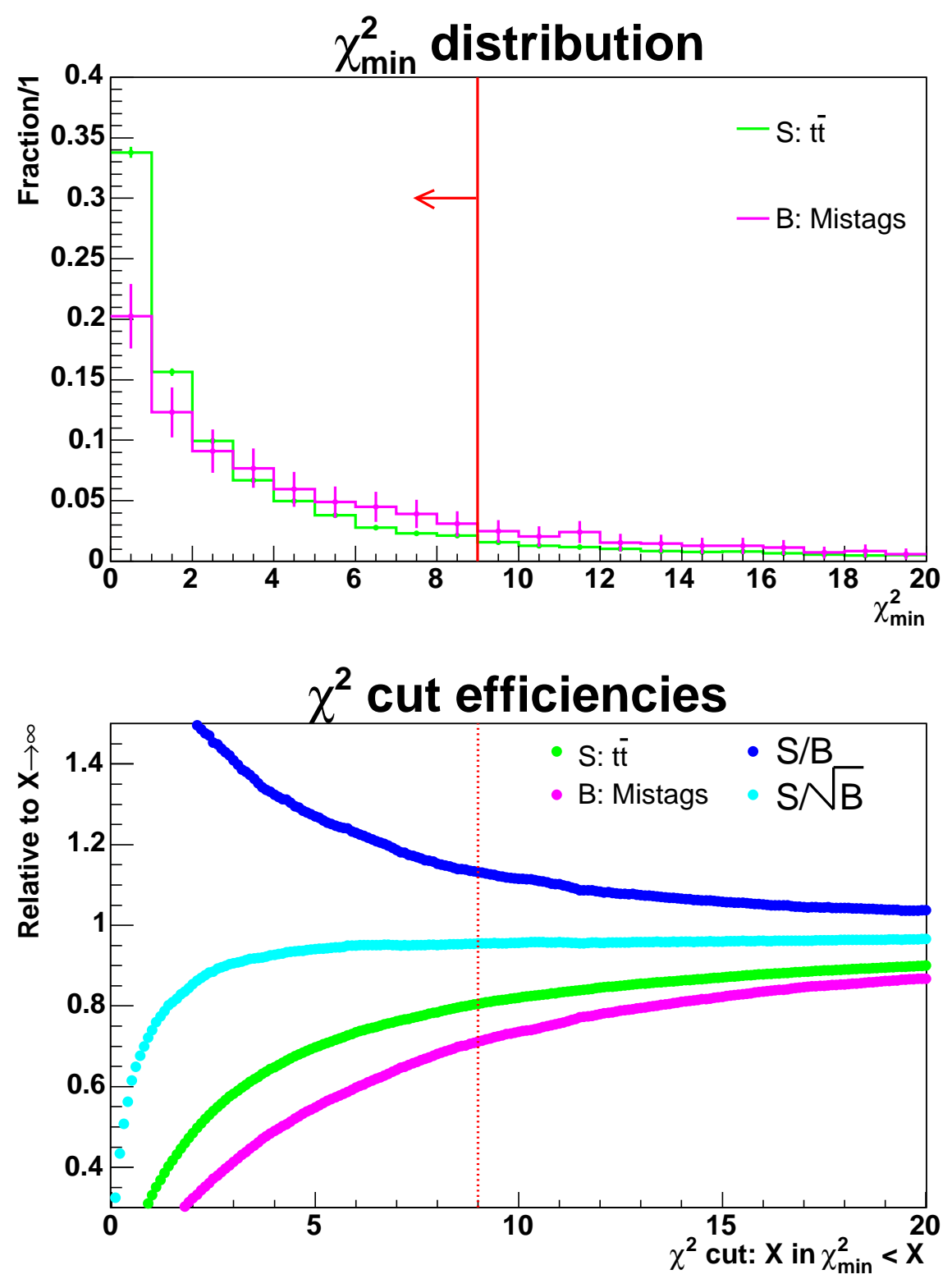

Figure 5.4: Top: the $\chi^{2}$ distributions of signal and background Monte Carlo samples. Bottom: The efficiency of the $\chi^{2}$ cut (relative to no cut) on signal and $W b \bar{b}$ background samples. Also shown are $S / B$ and $S / \sqrt{B}$, where $S$ and $B$ are the signal and background efficiencies, respectively. In both plots, the red line marks our applied cut $\chi_{\min }^{2}<9$. 


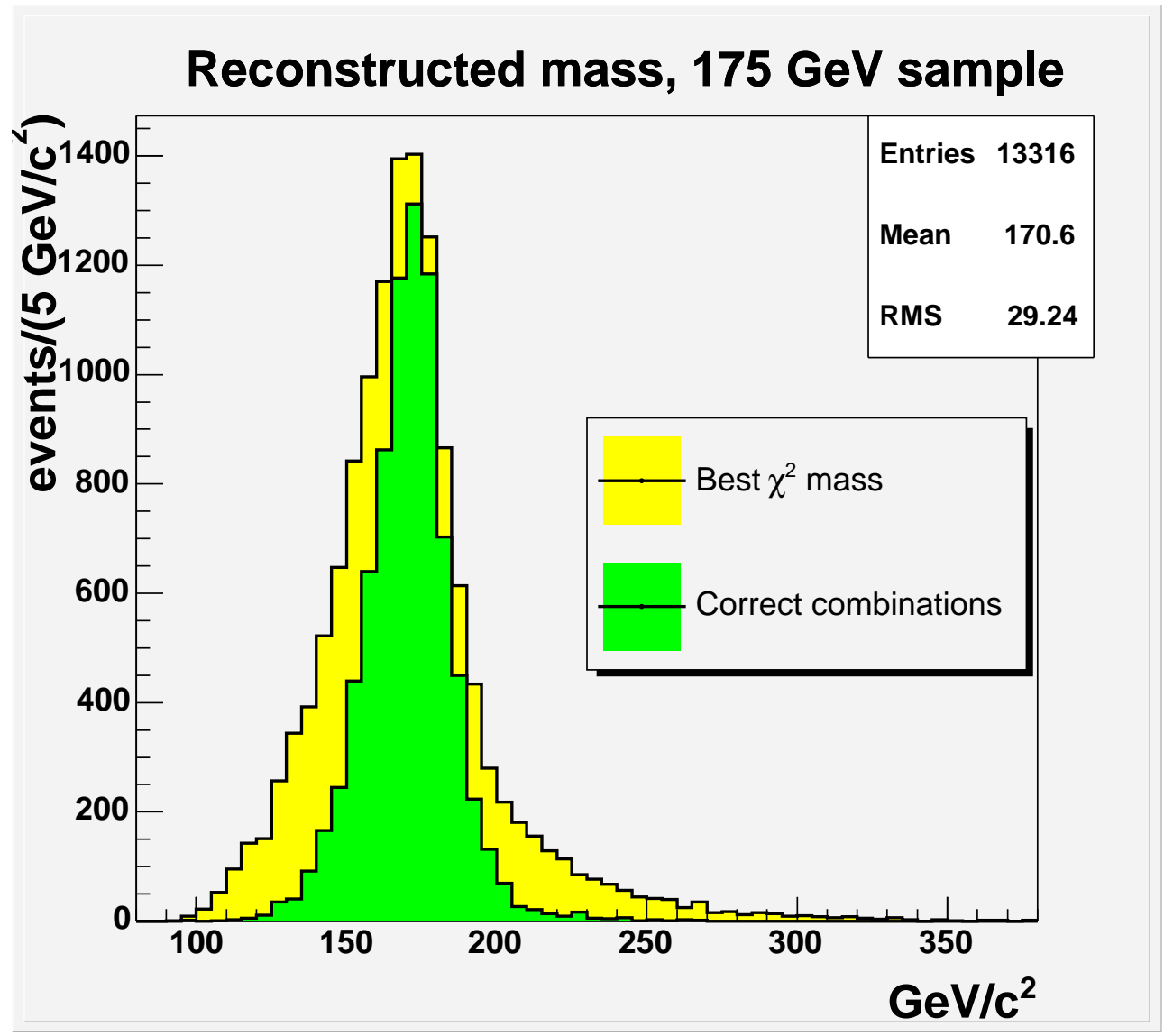

Figure 5.5: In yellow (wide histogram), the reconstructed mass distribution for the $175 \mathrm{GeV} / c^{2}$ HERWIG $t \bar{t}$ sample. Overlaid in green (narrow histogram) is the reconstructed mass distribution for the correct parton-jet assignment, in the subset of events for which the leading four jets are matched to the four partons from the $t \bar{t}$ decay. 


\section{Chapter 6}

\section{Extracting the Top Mass}

Given an observable quantity that depends strongly on the mass of the top quark, namely the reconstructed mass, we can measure the top mass in a group of events by comparing the distribution of reconstructed mass in those events to probability density functions with known dependence on the true top quark mass. In this section we describe that procedure.

Each event after selection contributes a single value to the reconstructed mass distribution. We use an unbinned likelihood fit, described in section 6.3, to measure the top mass. But first we need the probability density functions for reconstructed mass as a function of top mass, which are derived from Monte Carlo samples as described in section 6.1. Since we have background events in our sample that cannot a priori be distinguished from the signal, we need also p.d.f.'s for the background contribution. These are the subject of section 6.2 .

\subsection{Monte Carlo Signal Templates}

The distribution of reconstructed mass for a particular Monte Carlo sample is referred to as a template. Signal mass templates are produced using 29 Monte Carlo samples with the input top mass at $2.5-5 \mathrm{GeV} / c^{2}$ intervals from $130 \mathrm{GeV} / c^{2}$ to $230 \mathrm{GeV} / c^{2}$. Figure 6.1 shows nine selected mass templates built using the events that pass the event selection criteria $\left(E_{T}^{j 4}>8 \mathrm{GeV}\right)$ described in chapter 4 and the $\chi_{\min }^{2}<9$ cut described in chapter 5. In Figure 6.2 are shown the means, medians, and most probable values of all 29 mass templates as a function of the generated top mass, as well as their slopes from a 
linear fit.

From these templates we want to obtain p.d.f.'s that are a smoothly varying function of true top mass. We fit to an arbitrary parameterization that gives the probability to observe a particular reconstructed top mass given the true top mass. For a given true top mass, the parameterization consists of two Gaussians - intended to account for the well measured events - plus the integrand of the Gamma function - intended to account for events where the incorrect combination is chosen, or where the four leading jets do not match the four partons from the $t \bar{t}$ decay. The second Gaussian is not well motivated a priori, but was added to the parameterization in order to fit better the current generation of large statistics Monte Carlo samples. We assume that the 9 parameters necessary to specify this combination of functions themselves depend linearly on the true top mass, so that the full set of p.d.f.'s is specified by 18 parameters. This assumption is certainly arbitrary as well, but seems to be reasonable in the limited range of top masses we consider. Adding a quadratic term to the dependence of each parameter on the true top mass did not improve the quality of the fit. Thus the parameterization of the $t \bar{t}$ signal p.d.f. is as follows:

$$
\begin{aligned}
P_{\text {sig }}(m ; M)= & \alpha_{7} \cdot \frac{\alpha_{2}^{1+\alpha_{1}}}{\Gamma\left(1+\alpha_{1}\right)} \cdot\left(m-\alpha_{0}\right)^{\alpha_{1}} e^{-\alpha_{2}\left(m-\alpha_{0}\right)} \\
& +\alpha_{8} \cdot \frac{1}{\alpha_{4} \sqrt{2 \pi}} \cdot e^{\frac{-\left(m-\alpha_{3}\right)^{2}}{2 \alpha_{4}^{2}}} \\
& +\left(1-\alpha_{7}-\alpha_{8}\right) \cdot \frac{1}{\alpha_{6} \sqrt{2 \pi}} \cdot e^{\frac{-\left(m-\alpha_{5}\right)^{2}}{2 \alpha_{6}^{2}}}
\end{aligned}
$$

where

$$
\alpha_{i}=p_{i}+p_{i+9} \cdot(M-175)
$$

$M$ in $\mathrm{GeV} / c^{2}$ refers to the true top mass, and $m$ in $\mathrm{GeV} / c^{2}$ refers to the reconstructed top mass. Note that the p.d.f.'s are normalized so that, for a given true top mass $M$, the integral over all reconstructed top masses $m$ is unity.

A binned likelihood fit is used to extract the 18 parameter values. The full covariance matrix of uncertainties from this binned likelihood fit is saved and used to allow the parameters to vary in the final, unbinned likelihood fit to the data described in section 6.3. For the templates shown in Figures 6.1 and 6.2, the fit returns the parameters given in Table 6.1. Figure 6.3 shows the p.d.f.'s for true top mass between $130 \mathrm{GeV} / c^{2}$ and $230 \mathrm{GeV} / c^{2}$. The $\chi^{2}$ is calculated between the MC samples and the prediction from the fit, 


\section{CDF Run II Preliminary}
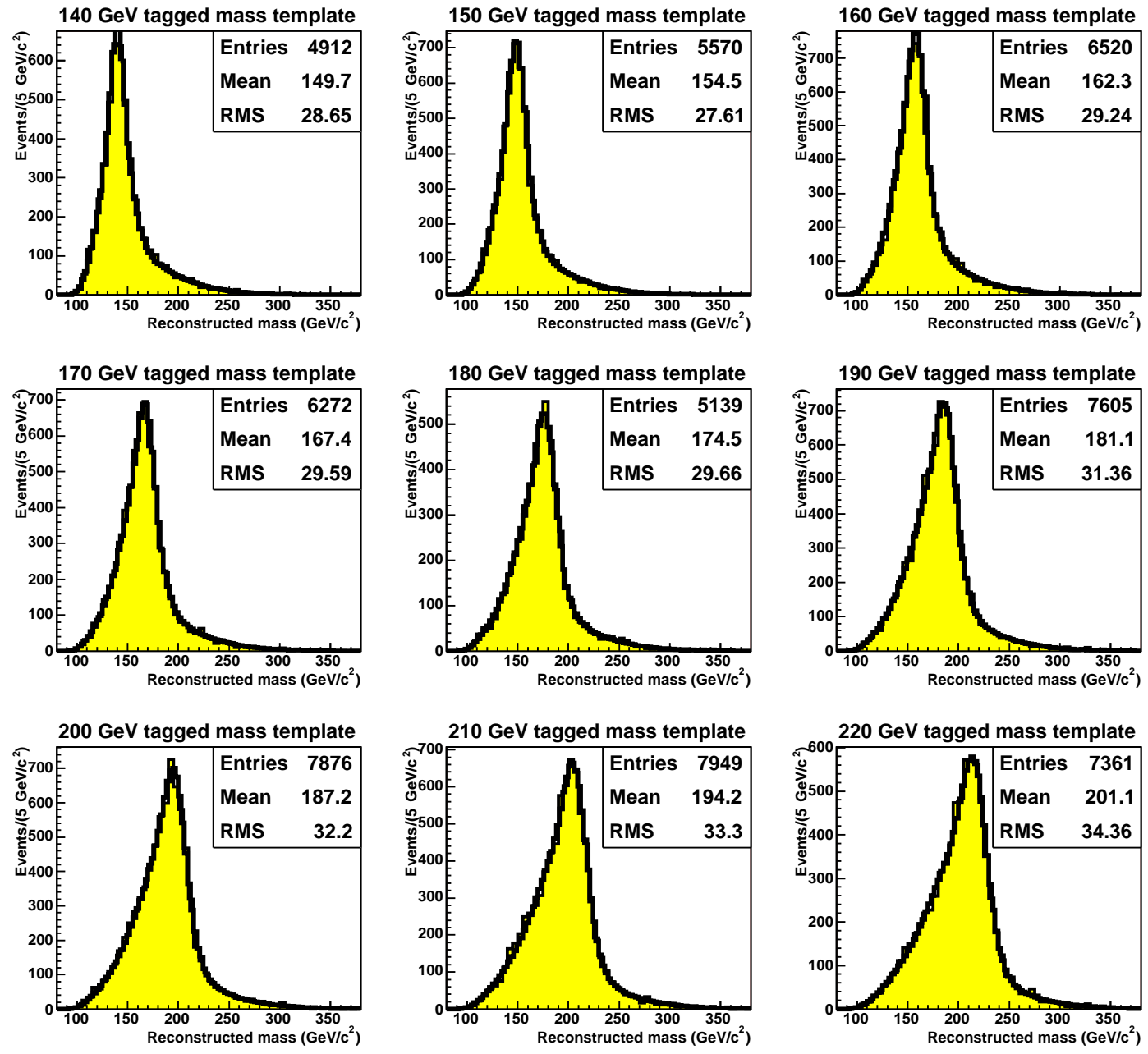

Figure 6.1: Selected Monte Carlo top mass templates with generated top mass between $140 \mathrm{GeV} / c^{2}$ and $220 \mathrm{GeV} / c^{2}$. Overlaid are curves from the parameterization whose form is given in Eq. 6.1.1 and whose fitted parameters are given in Table 6.1. 


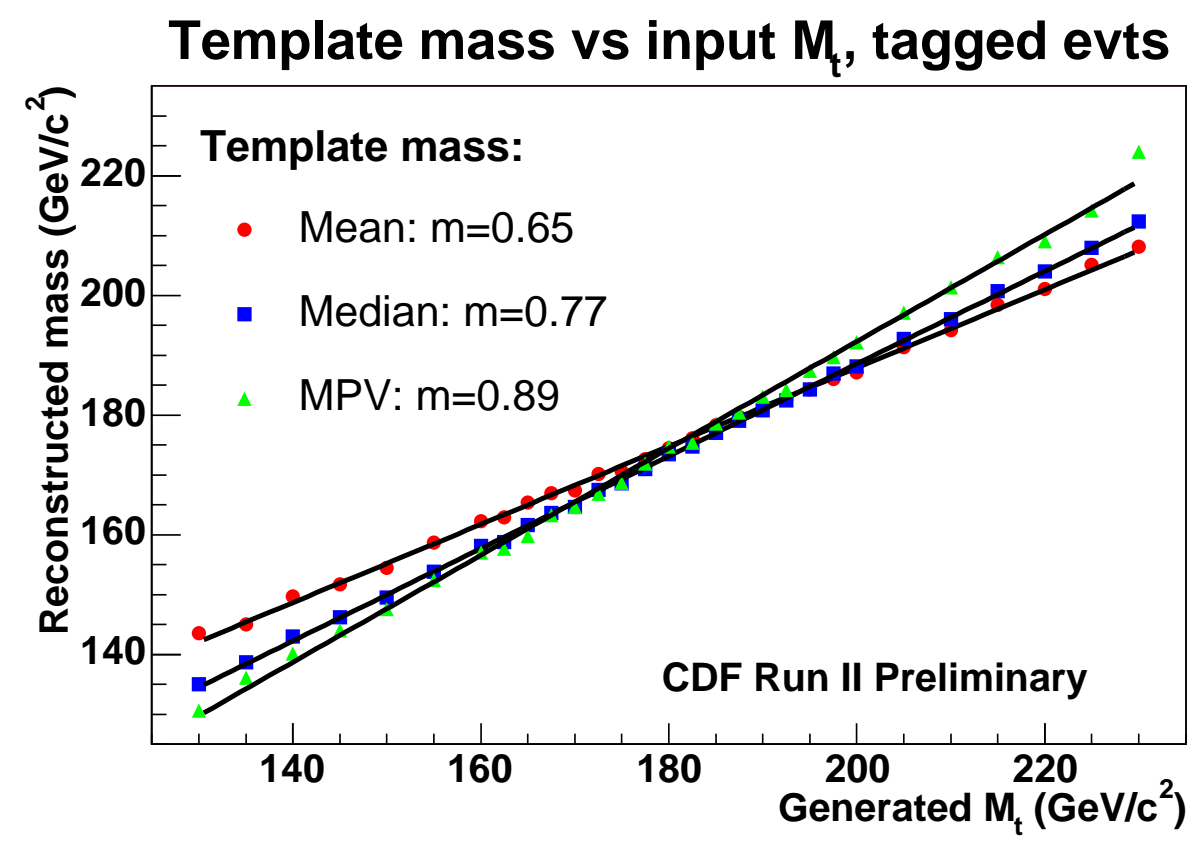

Figure 6.2: The means, medians, and most probable values of the $t \bar{t}$ signal mass templates are shown as a function of the generated top mass between $130 \mathrm{GeV} / c^{2}$ and $230 \mathrm{GeV} / c^{2}$. Each set of points is fitted to a line; the slopes are given in the legend.

after rebinning to ensure that each bin has at least five predicted events. The resulting $\chi^{2}$ is 1365 , with 1263 degrees of freedom, for a reduced $\chi^{2}$ of $1.08 .^{1}$

\subsection{Monte Carlo Background Template}

In modeling the background shape, which will be used in the final likelihood fit to reconstructed mass, we use Monte Carlo samples generated with Pythia, HERWIG, and ALPGEN. Samples corresponding to all processes listed in Table 4.5 were reconstructed using the procedure in chapter 5 , except $W W / W Z$ because of its very small contribution to the total background. $W c \bar{c}$ and $W c$ were shown to have the same reconstructed mass shape as $W b \bar{b}$ within statistics. Given the relatively small statistics available for those two processes, and the larger statistics available for $W b \bar{b}$, we have used the $W b \bar{b}$ shape for all three contributions, as well as the small $W W / W Z$ background.

\footnotetext{
${ }^{1}$ This corresponds to a very small probability for such a large number of degrees of freedom. So even with the addition of a second Gaussian in the parameterization, the limited power of our arbitrarily chosen function to describe the high-statistics templates is beginning to show.
} 
Table 6.1: The fitted parameters for the signal p.d.f.'s are given. The form of the parameterization is given in Equation 6.1.1.

\begin{tabular}{|c|c|}
\hline $\begin{array}{c}\text { Parameter } \\
\text { (see Eq. 6.1.1) }\end{array}$ & Fitted value \\
\hline$p_{0}$ & $87.9 \pm 0.5$ \\
$p_{1}$ & $3.32 \pm 0.08$ \\
$p_{2}$ & $0.0488 \pm 0.0006$ \\
$p_{3}$ & $173.3 \pm 0.2$ \\
$p_{4}$ & $8.2 \pm 0.2$ \\
$p_{5}$ & $165.1 \pm 0.3$ \\
$p_{6}$ & $17.2 \pm 0.2$ \\
$p_{7}$ & $0.411 \pm 0.006$ \\
$p_{8}$ & $0.179 \pm 0.009$ \\
$p_{9}$ & $-0.15 \pm 0.02$ \\
$p_{10}$ & $0.025 \pm 0.003$ \\
$p_{11}$ & $-2 \pm 2) \times 10^{-5}$ \\
$p_{12}$ & $0.956 \pm 0.005$ \\
$p_{13}$ & $0.077 \pm 0.005$ \\
$p_{14}$ & $0.748 \pm 0.009$ \\
$p_{15}$ & $0.127 \pm 0.005$ \\
$p_{16}$ & $-0.00035 \pm 0.00014$ \\
$p_{17}$ & $0.0015 \pm 0.0002$ \\
\hline$\chi^{2} /$ n.d.o.f. & $1365 / 1263$ \\
\hline
\end{tabular}

\section{CDF Run II Preliminary}
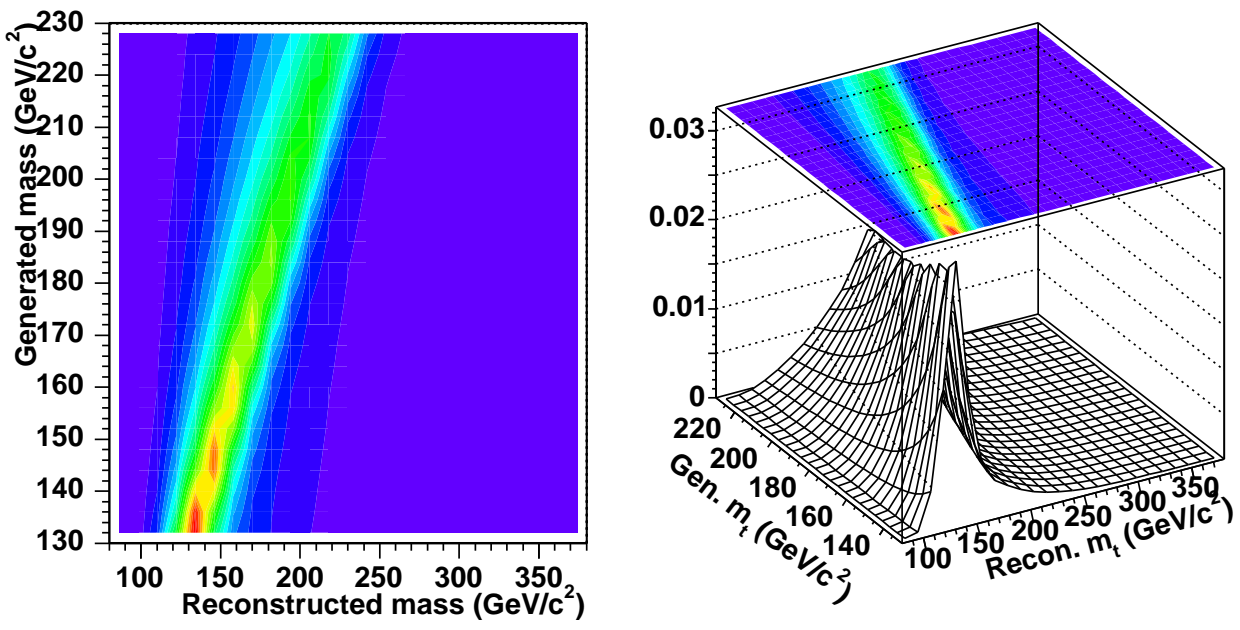

Figure 6.3: Visualizations of the fitted probability density functions for $130 \mathrm{GeV} / c^{2}<$ $\mathrm{M}_{\text {top }}<230 \mathrm{GeV} / c^{2}$. 
Large single-top samples are available in the $s$ channel and $t$ channel separately. The $s$ channel and the $t$ channel contribute $60 \%$ and $40 \%$ of the single top background with our selection, respectively. Since the two channels have different kinematics, we expect the reconstructed mass distributions to be different. Even though the contributions are small, we make use of our ample statistics and treat the two channels as separate processes.

The most elusive background comes from QCD events, i.e. events with no real $W$ to produce the isolated lepton and $E_{T}$. We looked at events in the data with non-isolated leptons, which are expected to be enriched in this type of background, but kinematically similar to our signal events. Within the limited statistics available, Figure 6.4 (left) shows that the reconstructed mass distribution of the QCD-enriched events is consistent with that of $W+$ jets events. In order to improve the statistics, we also compare the $W+$ jets template with a QCD-enriched template made without using a $\chi^{2}$ cut and using all pre-tagged events where the most probable heavy-flavor jet ${ }^{2}$ is required to be assigned to a $b$ parton. These templates also appear to be very similar (Figure 6.4, right). A flattened KS test gives a probability of $6.5 \%$ for the first plot, and $50.7 \%$ for the second. ${ }^{3}$ Given these similarities, we use the shape of the $W+$ jets reconstructed mass template for the expected contribution from QCD. ${ }^{4}$

Thus we consider reconstructed mass distributions from the following processes:

- $W+$ jets (mistags)

- $W b \bar{b}$

- Single top in the $s$ channel

- Single top in the $t$ channel

The reconstructed mass template for the $W+$ jets (mistag) sample is determined using a $W+4$ parton sample, where the $E_{T}$ cut on generated partons is $8 \mathrm{GeV}$. The $b$ tags found in Monte Carlo are not used for this sample (and this sample alone). This is because the Monte Carlo $b$ tagger is not trusted to model well the rate or kinematic

\footnotetext{
${ }^{2}$ We use an algorithm that determines the heavy flavor probability using information about tracks inside the jets.

${ }^{3}$ In the flattened KS test, the KS probability is normalized using an ensemble of KS distances measured in sets of events randomly drawn from the large-statistics sample.

${ }^{4}$ This background could be controlled better. One idea is to apply additional cuts, such as a $\Delta \phi$ cut between $E_{T}$ and the leading jet, that would diminish the relative contribution of this poorly understood background.
} 

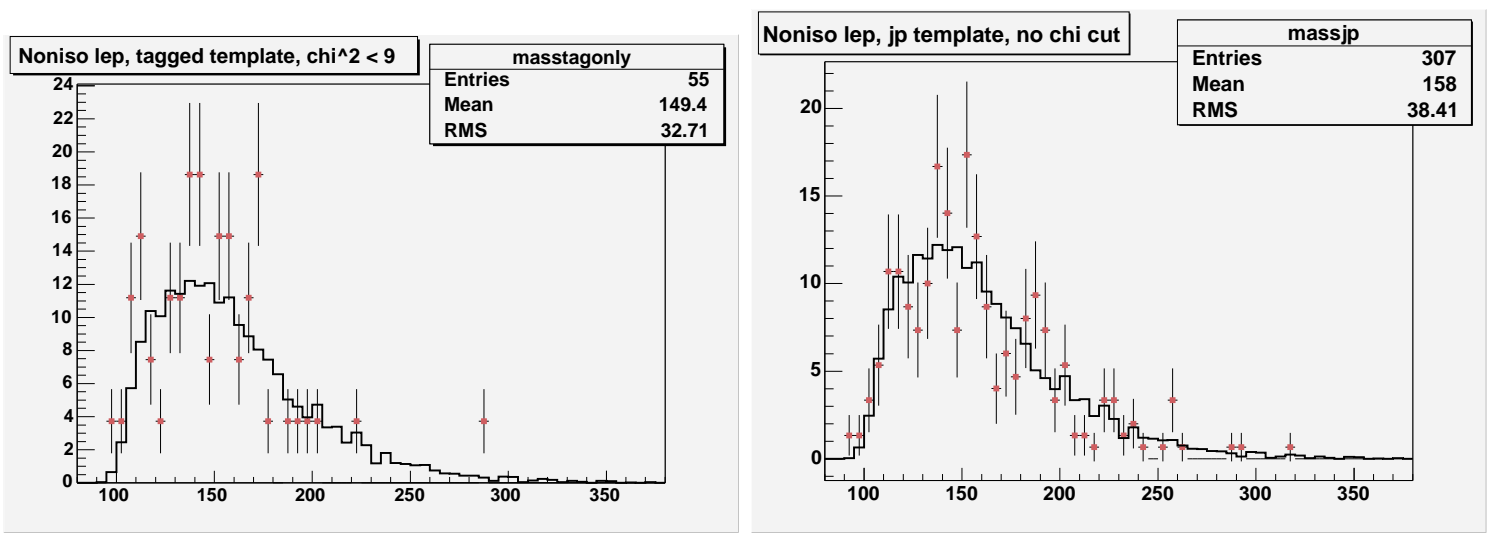

Figure 6.4: Comparison between $W+$ jets templates (histograms) and data events with nonisolated leptons (points). The latter are thought to be enriched in non- $W$ background. At left, the points are the default template, including the cut $\chi^{2}<9$. At right, the points are given improved statistics, both by eliminating the $\chi^{2}$ cut, and by using the full pre-tagged sample, with the most likely heavy flavor jet identified as a $b$.

dependences of fake tags. Instead, the mistag matrix is queried for each jet to determine the probability of getting a negative tag for that $E_{T}, \phi, \eta, N_{t r k}$, and $\Sigma E_{T}$. That probability is multiplied by 1.2 to account for an asymmetry in the $L_{x y}$ of mistags, and the result is considered to be the probability to get a positive mistag on that jet. Next we consider each possible configuration of tags in the event. There are four single-tag configurations and six double-tag configurations. For each one we determine the best tag-consistent assignment of jets to partons, and fill the mass template with a weight corresponding to the probability of observing that tag configuration. The result is a weighted template for the mistag background. The reconstructed mass template for the $W b \bar{b}$ and similar backgrounds is made using the $W b \bar{b}+2$ parton sample exactly as for the signal samples, and the single top templates are produced in the same way.

In order to estimate the number of background events in our mass fitting sample, we must account for the effect of the $\chi_{\min }^{2}<9$ cut. The efficiency of this cut for background samples is around 60\%-70\% (see Table 6.2), taken directly from the fraction of events that pass the cut in our Monte Carlo samples. In the case of the mistag background, the uncertainty is inflated to account for possible differences in efficiency between mistag and QCD events. In the QCD-enriched data sample with non-isolated leptons, 55/68 tagged events survive the $\chi^{2}$ cut, for an efficiency of $81 \pm 5 \%$. We also have a small effect due to the requirement that a tag be on one of the leading four jets instead of on any jet in the 
Table 6.2: Top: the number of background events in each combined background sample. Middle: the efficiency of $\chi_{\min }^{2}<9$ and requiring the tag on the leading four jets for each mass template. Bottom: the number of background events used in the mass fitting.

\begin{tabular}{|c|c|c|c|c|}
\hline \multicolumn{5}{|c|}{ Background events without $\chi_{\min }^{2}<9$} \\
\hline Mass Template Source & \multicolumn{2}{|c|}{ Background Source } & $W+3.5$ jets & $W+\geq 4$ jets \\
\hline$W+$ jets (mistags) & \multirow{2}{*}{\multicolumn{2}{|c|}{$\begin{array}{c}\text { mistags, QCD } \\
W b \bar{b}, W c \bar{c}, W c, W W / W Z\end{array}$}} & \multirow{2}{*}{$\begin{array}{l}3.3 \pm 0.6 \\
2.1 \pm 0.6\end{array}$} & $2.9 \pm 0.6$ \\
\hline$W b \bar{b}$ & & & & $1.1 \pm 0.4$ \\
\hline Single top: s channel & & & $0.18 \pm 0.03$ & $0.13 \pm 0.02$ \\
\hline Single top: t channel & & & $0.12 \pm 0.02$ & $0.06 \pm 0.02$ \\
\hline \multicolumn{5}{|c|}{ Efficiency of cuts } \\
\hline Mass template & \multicolumn{2}{|c|}{$\chi_{\min }^{2}<9$} & \multicolumn{2}{|c|}{ Tag on leading 4 jets } \\
\hline source & $W+3.5$ jets & $W+\geq 4$ jets & \multicolumn{2}{|c|}{$W+\geq 4$ jets } \\
\hline$W+$ jets (mistags) & $0.716 \pm 0.070$ & $0.707 \pm 0.070$ & \multicolumn{2}{|c|}{$0.973 \pm 0.010$} \\
\hline$W b \bar{b}$ & $0.668 \pm 0.011$ & $0.692 \pm 0.012$ & \multicolumn{2}{|c|}{$0.979 \pm 0.004$} \\
\hline Single top: s channel & $0.598 \pm 0.016$ & $0.665 \pm 0.018$ & \multicolumn{2}{|c|}{$0.994 \pm 0.003$} \\
\hline Single top: t channel & $0.793 \pm 0.026$ & $0.564 \pm 0.040$ & \multicolumn{2}{|c|}{$0.987 \pm 0.009$} \\
\hline \multicolumn{5}{|c|}{ Background events with $\chi_{\min }^{2}<9$} \\
\hline Mass Template Source & \multicolumn{2}{|c|}{ Background Source } & $W+3.5$ jets & $W+\geq 4$ jets \\
\hline \multirow{2}{*}{$\begin{array}{c}W+\text { jets (mistags) } \\
W b \bar{b}\end{array}$} & \multirow{2}{*}{\multicolumn{2}{|c|}{$\begin{array}{c}\text { mistags, QCD } \\
W b \bar{b}, W c \bar{c}, W c, W W / W Z\end{array}$}} & $2.4 \pm 0.5$ & $2.0 \pm 0.5$ \\
\hline & & & $1.4 \pm 0.4$ & $0.7 \pm 0.3$ \\
\hline \multirow{2}{*}{\multicolumn{3}{|c|}{$\begin{array}{l}\text { Single top: s channel } \\
\text { Single top: t channel }\end{array}$}} & $0.11 \pm 0.02$ & $0.09 \pm 0.01$ \\
\hline & & & $0.10 \pm 0.01$ & $0.03 \pm 0.01$ \\
\hline \multicolumn{3}{|l|}{ Total } & $4.0 \pm 0.6$ & $2.9 \pm 0.5$ \\
\hline & & & \multicolumn{2}{|c|}{$6.8 \pm 1.2$} \\
\hline
\end{tabular}

event with $E_{T}>15 \mathrm{GeV}$. The efficiency for this requirement is also calculated from MC, and is given in Table 6.2 for $\geq 4$-jet events; this requirement is by definition $100 \%$ efficient for $W+3.5$-jet events.

We do not allow each background contribution to vary independently in the final likelihood fit. Instead, the eight contributions listed in Table 6.2 (3.5- and 4-jet contributions from each of four processes) are added with appropriate weights to create a combined background template, and the normalization of the combined shape is permitted to vary in the final likelihood fit (see section 6.3). Since the quantity that varies in the likelihood fit is the expectation value of the number of background events in the sample, we do not need to account for any counting error. The total number of background events is estimated to be $4.0 \pm 0.6$ in the $W+3.5$ jet bin and $2.9 \pm 0.5$ in the $W+\geq 4$ jet bin. The total background estimate for $W+\geq 3.5$ jets is $6.8 \pm 1.2$. Complete details are given in Table 6.2. 
CDF Run II Preliminary
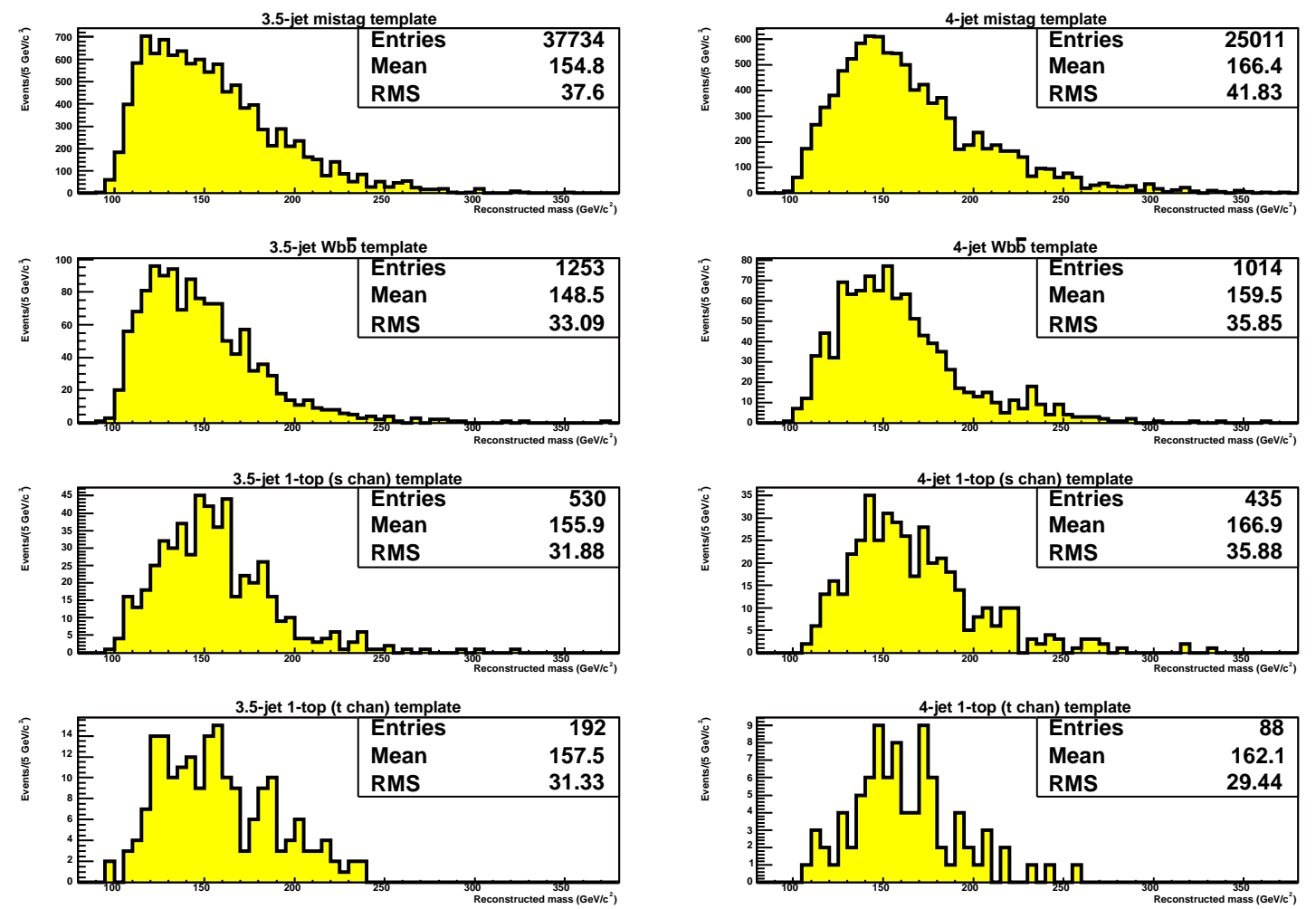

Figure 6.5: Reconstructed mass distributions for different background sources. On the left are the 3.5-jet templates, and on the right the 4-jet templates. From top to bottom: $W+$ jets (mistags), $W b \bar{b}$, single top ( $s$ channel), and single top ( $t$ channel).

The mass templates for each process and the combined background mass template are shown in Figures 6.5 and 6.6. The systematic uncertainty due to background shape modeling will be obtained by comparing the fit masses when the mass template of each separate background process is used.

Finally, we determine the p.d.f. for the background reconstructed mass using a parameterization similar in spirit to that of the signal, but much simpler. First, there is obviously no dependence on top mass. Second, no narrow Gaussian peak is expected, so the full shape is modeled by the integrand of the Gamma function. Specifically,

$$
P_{b g}(m)=\frac{p_{2}^{1+p_{1}}}{\Gamma\left(1+p_{1}\right)} \cdot\left(m-p_{0}\right)^{p_{1}} e^{-p_{2}\left(m-p_{0}\right)}
$$

where $m$ in $\mathrm{GeV} / c^{2}$ is the reconstructed top mass. For our combined reconstructed mass template, shown in Figure 6.6, the fitted parameters are given in Table 6.3. After rebinning 
Table 6.3: The fitted parameters for the background p.d.f. are given. The form of the parameterization is given in Equation 6.2.1.

\begin{tabular}{|c|c|}
\hline $\begin{array}{c}\text { Parameter } \\
\text { (see Eq. 6.2.1) }\end{array}$ & Fitted value \\
\hline$p_{0}$ & $94 \pm 4$ \\
$p_{1}$ & $1.8 \pm 0.5$ \\
$p_{2}$ & $0.045 \pm 0.006$ \\
\hline$\chi^{2} /$ n.d.o.f. & $12 / 34$ \\
\hline
\end{tabular}

so that each bin has at least five predicted events, the $\chi^{2}$ for the fit is 12 , with 34 degrees of freedom. ${ }^{5}$

\subsection{Likelihood}

To measure the top mass, we compare the reconstructed mass distribution from data to a sum of p.d.f.'s from signal (section 6.1) and background (section 6.2) Monte Carlo using an unbinned extended likelihood fit.

There are 18 parameters in the parameterization of the signal p.d.f.'s as a function of $\mathrm{M}_{\mathrm{top}}$, and 3 in the parameterization of the background p.d.f. All 21 of these are permitted to vary in the likelihood fit; they are constrained by the covariance matrices output from their independent binned likelihood fits.

The extended likelihood fit involves parameters for the expectation values of the number of signal and background events. It contains terms that sum over the possible Poisson fluctuations of these quantities. In the absence of prior constraints, the best fit expectation values are the same as the best fit for the number of signal or background events in the sample, but this is no longer the case when for example the expected number of background events is constrained. The $22^{\text {nd }}$ parameter in the unbinned likelihood fit is the expectation value for the number of signal events, and the $23^{\text {rd }}$ parameter is the expectation value for the number of background events. The former is unconstrained, and the latter is constrained by a simple Gaussian. We also perform fits where the background fraction is not constrained. In this case (and technically even in the constrained case), the

\footnotetext{
${ }^{5}$ The bin-by-bin uncertainties for the combined template may be incorrect due to complicated uncertainties on the mistag shape that are not treated absolutely correctly, leading to an incorrect $\chi^{2}$. The binned likelihood fit assumes a histogram filled with a constant weight.
} 


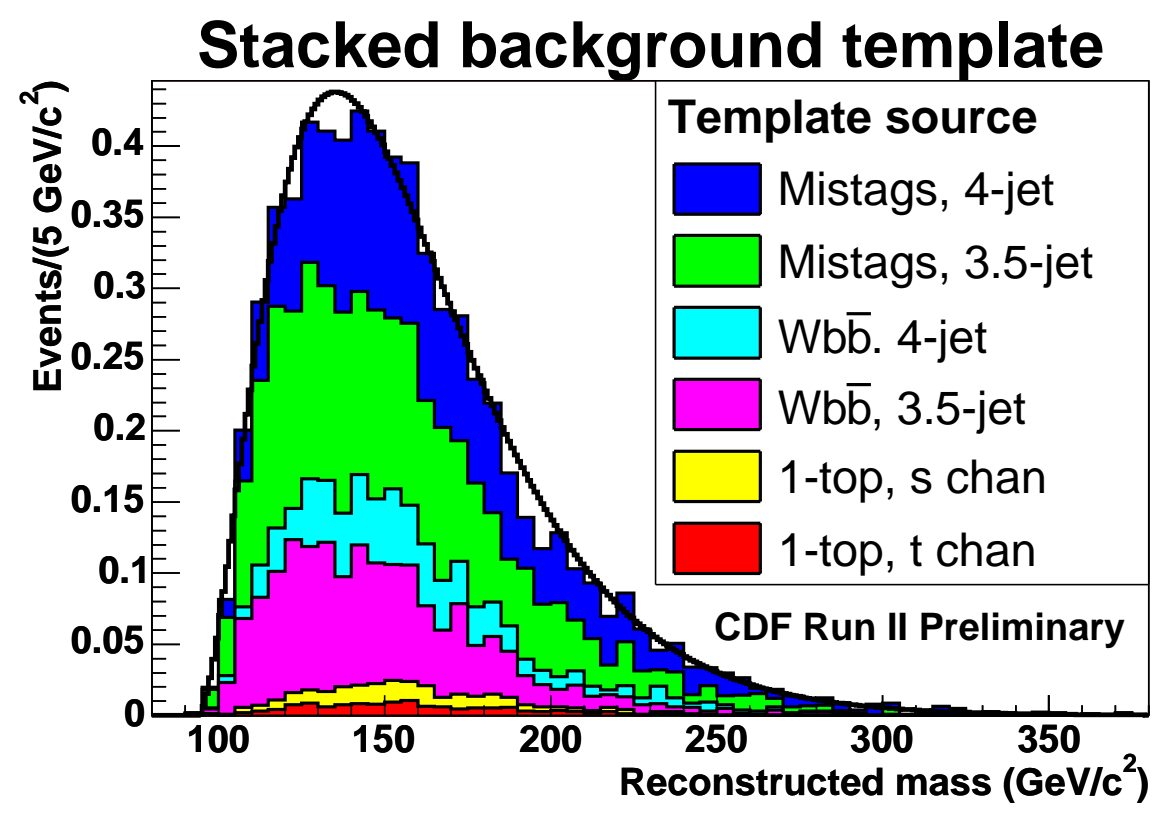

Figure 6.6: Reconstructed mass distribution of the combined backgrounds. The contributions from different background processes are stacked. Overlaid is the fitted curve (see Eq. 6.2.1) with parameters given in Table 6.3.

best fit may have an unphysical background fraction, i.e. $f_{b g}<0$ or $f_{b g}>1$.

Finally, the true top mass is the final (and most interesting!) parameter. It has no constraint. Its initial value is $175 \mathrm{GeV} / c^{2}$. The likelihood is maximized with respect to all 24 parameters using the MINUIT package.

The likelihood is given by:

$$
\begin{aligned}
\mathcal{L} & =\mathcal{L}_{\text {shape }} \times \mathcal{L}_{\text {bg }} \times \mathcal{L}_{\text {params }} ; \\
\mathcal{L}_{\text {shape }} & =\frac{e^{-\left(n_{s}+n_{b}\right)}\left(n_{s}+n_{b}\right)^{N}}{N !} \prod_{i=1}^{N} \frac{n_{s} P_{\text {sig }}\left(m_{i} ; \mathrm{M}_{\text {top }}\right)+n_{b} P_{b}\left(m_{i}\right)}{n_{s}+n_{b}} ; \\
-\ln \mathcal{L}_{b g} & =\frac{\left(n_{b}-n_{b}^{e x p}\right)^{2}}{2 \sigma_{n_{b}}^{2}} ; \\
-\ln \mathcal{L}_{\text {params }} & =\sum_{i=0}^{17} \sum_{j=0}^{17} \frac{1}{2}\left(\delta p_{i}^{\text {sig }} C_{i j}^{\text {sig }} \delta p_{j}^{\text {sig }}\right)+\sum_{i=0}^{2} \sum_{j=0}^{2} \frac{1}{2}\left(\delta p_{i}^{b} C_{i j}^{b} \delta p_{j}^{b}\right) ;
\end{aligned}
$$

where the last two terms are given as the negative log likelihood for simplicity. $N$ is the total number of events observed in the data, $m_{i}$ is the reconstructed mass of the $i$ th event, $P_{s i g}$ is the signal p.d.f. defined in Equation 6.1.1, $P_{b}$ is the background p.d.f. defined in Equation 6.2.1, $\mathrm{M}_{\mathrm{top}}$ is the value of the true top mass parameter, $C$ is the inverted error 
matrix from either of the binned likelihood fits for the p.d.f. parameters, and $\delta p_{i}$ is the difference between the fitted $i$ th parameter and the input, for signal or background.

We note that since we fit for both $\mathrm{M}_{\text {top }}$ and $n_{s}$, the expectation value of the number of signal events, we could extract the top mass and the $t \bar{t}$ cross-section simultaneously (given the efficiency of our $\chi^{2}$ cut on signal events, plus various other quantities already available). It would be interesting to do this exercise and see the cross-section value we observe, as well as its correlation with the top mass.

\subsection{Likelihood Checks and Statistical Uncertainty}

We check the fitting procedure on our Monte Carlo samples. Checking whether the likelihood fit returns the correct mass for a wide range of the input top masses is performed by comparing the fit mass using pseudoexperiments against the input top mass. See Appendix A for details on the generation of pseudoexperiments. We use 28-event pseudoexperiments, with a background constraint of $6.8 \pm 1.2$ events. As illustrated in Figure 6.7, the slope of the linear fit between the input and fit top mass is consistent with one. The centers and widths of the associated pull distributions are checked in Figure 6.8. The pull widths are consistently larger than one. We believe this is an unavoidable problem with trying to describe non-Gaussian errors with a single " $1 \sigma$ " uncertainty. However, we would like our quoted $\pm 1 \sigma$ statistical uncertainties to cover the true mass value (neglecting systematic effects) $68 \%$ of the time. To achieve this coverage, we scale the raw uncertainties by a factor of 1.065. See Appendix B for more details.

The raw expected statistical uncertainty (before scaling the errors as described in Appendix B) on the top mass from 28 top candidate events with $6.8 \pm 1.2$ expected background events is estimated from pseudoexperiments. Figure 6.9 shows the raw expected uncertainty as a function of the top mass for both the default measurement $(\geq 3.5$-jet events) and the 4-jet-only measurement. The default measurement is expected to perform slightly better across the board. For the measurement using 4-jet events only, the signal and background parameterizations are determined using only 4-jet events, and the 4-jet background composition and normalization are used in the fit. 


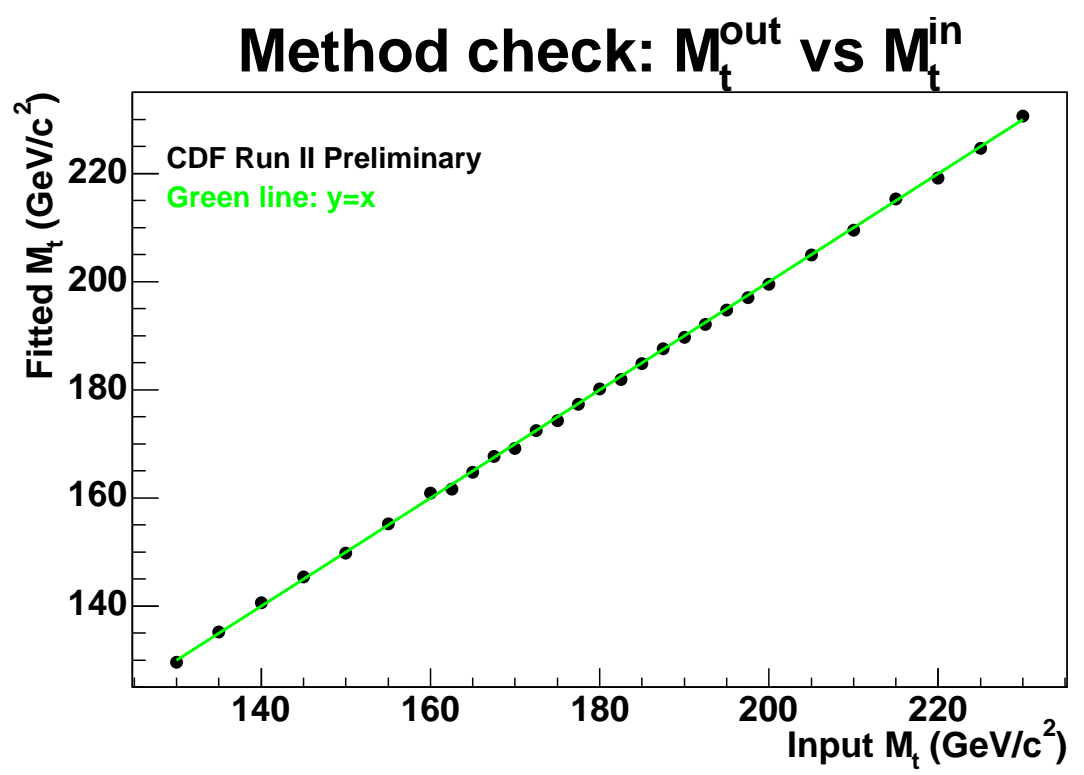

Figure 6.7: The returned (fit) top mass as a function of the input top mass. The line is drawn with slope of one. Pseudoexperiments with 28 events each were performed, and the median of those pseudoexperiments is plotted here.
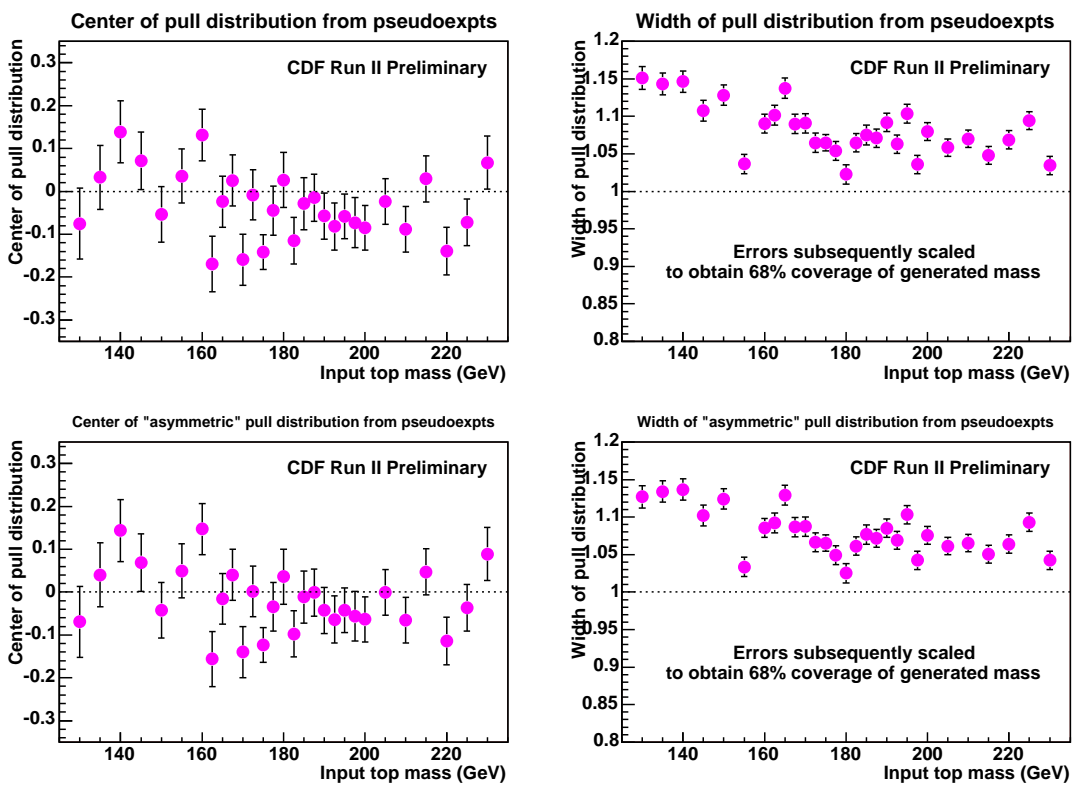

Figure 6.8: The center (left) and the width (right) of the mass pull distribution from 28event pseudoexperiments as a function of the input top mass. Top plots use a symmetrized error, while the bottom plots make use of the reported asymmetric errors. 

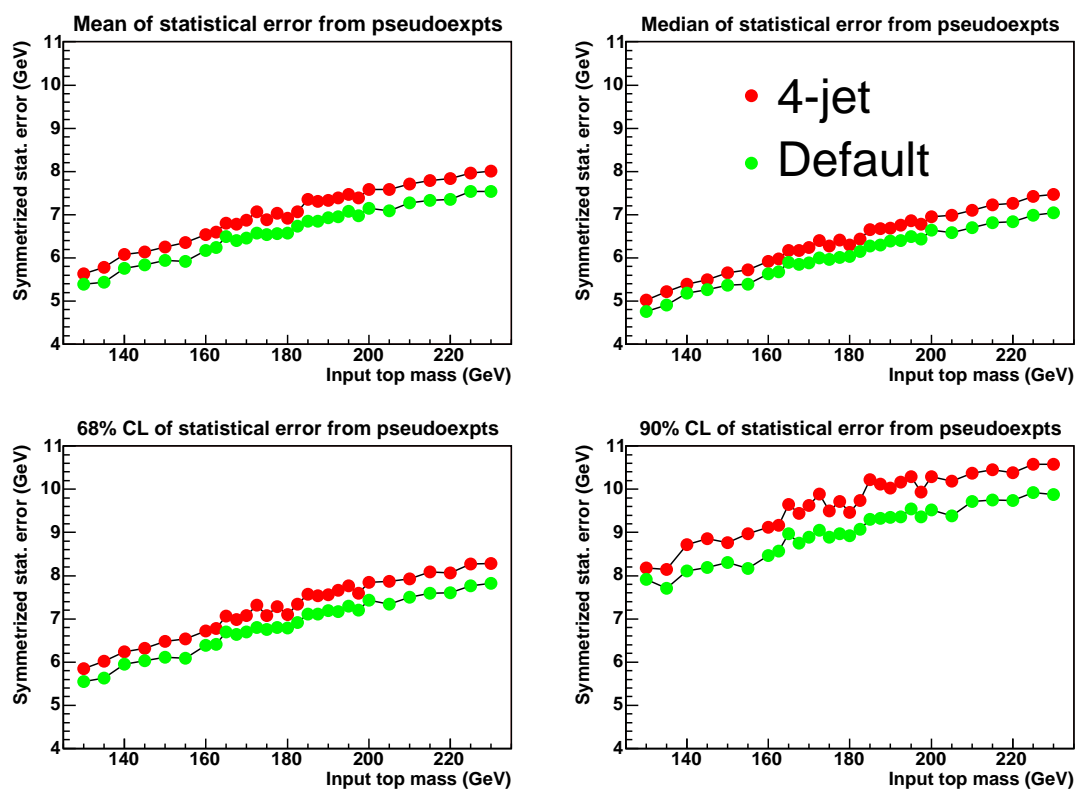

Figure 6.9: The estimated raw statistical uncertainty as a function of the input top mass using mean, median, 68\% CL, and 90\% CL. From 10,000 28-event pseudoexperiments. The green (lower) curve is for the default measurement ( $\geq 3.5$-jet events), and the red (upper) curve is for the measurement using only 4 -jet events (19-event pseudoexperiments). 


\section{Chapter 7}

\section{Results from the data}

Figure 7.1 (top) shows the reconstructed mass distribution from the data with $\geq 3.5$ jets, compared to a combination of the parameterized signal template at the fit mass and the combined background Monte Carlo template when the background normalization is constrained. The likelihood curve as a function of top mass is also shown in the inset. The extracted top mass is

$$
\mathrm{M}_{\text {top }}=174.9_{-7.2}^{+6.7} \text { (stat.) } \mathrm{GeV} / c^{2} \text {. }
$$

We check the KS probability for the data against the fitted signal and background templates. Normalizing the KS distance using sets of events drawn from the fitted templates, we find a KS probability of $21.9 \%$ for the data. When a finer binning is used $\left(3 \mathrm{GeV} / c^{2}\right.$ instead of $15 \mathrm{GeV} / c^{2}$ ), we find a probability of $34.4 \%$.

We also perform the fit without the background constraint as a check. In this case, shown in the bottom plot of Figure 7.1, the fitted mass, with raw statistical uncertainty, is

$$
\mathrm{M}_{\text {top }}=177.7_{-8.3}^{+9.2} \text { (stat.) } \mathrm{GeV} / c^{2} \text {. }
$$

In this case the normalized KS probabilities are $76.4 \%$ for $15 \mathrm{GeV} / c^{2}$ binning, and $89.9 \%$ for $3 \mathrm{GeV} / c^{2}$ binning. The number of background events favored by the fitter in the unconstrained fit is $16.6_{-7.3}^{+8.2}$, more than one sigma higher than the estimate of $6.8 \pm 1.2$.

After scaling the statistical uncertainty to achieve $68 \%$ coverage of the true mass value, as described in Appendix B, the default measurement becomes

$$
\mathrm{M}_{\text {top }}=174.9_{-7.7}^{+7.1} \text { (stat.) } \mathrm{GeV} / c^{2} \text {. }
$$


Table 7.1: Results of likelihood fit with and without background constraint. The measurement in bold is the primary result for this analysis. In the last column, the statistical uncertainties are scaled to provide $68 \%$ coverage of the true mass value (see Appendix B).

\begin{tabular}{|c|c|c|c|c|}
\hline Category & \multicolumn{2}{|c|}{ Background (events) } & \multirow{2}{*}{$\mathrm{M}_{\text {top }}\left(\mathrm{GeV} / c^{2}\right)$} & $\begin{array}{c}\mathrm{M}_{\text {top }}\left(\mathrm{GeV} / c^{2}\right) \\
\text { (scaled errors) }\end{array}$ \\
\cline { 2 - 5 } & input & output & & $\mathbf{1 7 4 . 9}_{-\mathbf{7 . 7}}^{+\mathbf{7 . 1}}$ \\
\hline \hline$W+\geq 3.5$ jets & $6.8 \pm 1.2$ & $7.1 \pm 1.2$ & $174.9_{-7.2}^{+6.7}$ & \\
\cline { 2 - 5 } & no constraint & $16.6_{-7.3}^{+8.2}$ & $177.7_{-8.3}^{+9.2}$ & \\
\hline \hline$W+\geq 4$ jets & $2.9 \pm 0.5$ & $2.9 \pm 0.5$ & $179.4 \pm 6.2$ & \\
\cline { 2 - 5 } & no constraint & $3.2_{-5.5}^{+6.5}$ & $179.5_{-6.2}^{+6.6}$ & \\
\hline
\end{tabular}

The necessary scaling has been determined only for the default measurement; all other fits are reported with raw uncertainties only. The fitted top mass and background values are summarized in Table 7.1.

We check that the statistical uncertainty seen in the data is consistent with expectations for our measured mass. Figure 7.2 shows the expected positive and negative uncertainty distributions for an input top mass of $175 \mathrm{GeV} / c^{2}$, where the arrows indicate the statistical uncertainties in the data. About $66 \%$ of the positive errors are smaller than our observed positive error of $+7.1 \mathrm{GeV} / c^{2}$, and about $80 \%$ of the negative errors are smaller (in magnitude) than our observed negative error of $-7.7 \mathrm{GeV} / c^{2}$. Figure 7.3 shows the same quantities in two dimensions so that the correlations are apparent. In Figures 7.2 and 7.3 , the scaled uncertainties are used.

Finally, we perform the 4-jet likelihood fit to the 19 such events in the data. The extracted top masses with and without the background constraint are

$$
\mathrm{M}_{\text {top }}=179.4 \pm 6.2 \text { (stat.) } \mathrm{GeV} / c^{2}
$$

and

$$
\mathrm{M}_{\mathrm{top}}=179.5_{-6.2}^{+6.6} \text { (stat.) } \mathrm{GeV} / c^{2}
$$

respectively. The reconstructed mass distribution and the fit results are shown in Figure 7.4 and Table 7.1. Although the statistical uncertainty on the 4 -jet sample happens to be smaller than that of the 3.5-jet sample, the 3.5-jet result is the primary measurement, and the 4 -jet result is considered a check. 

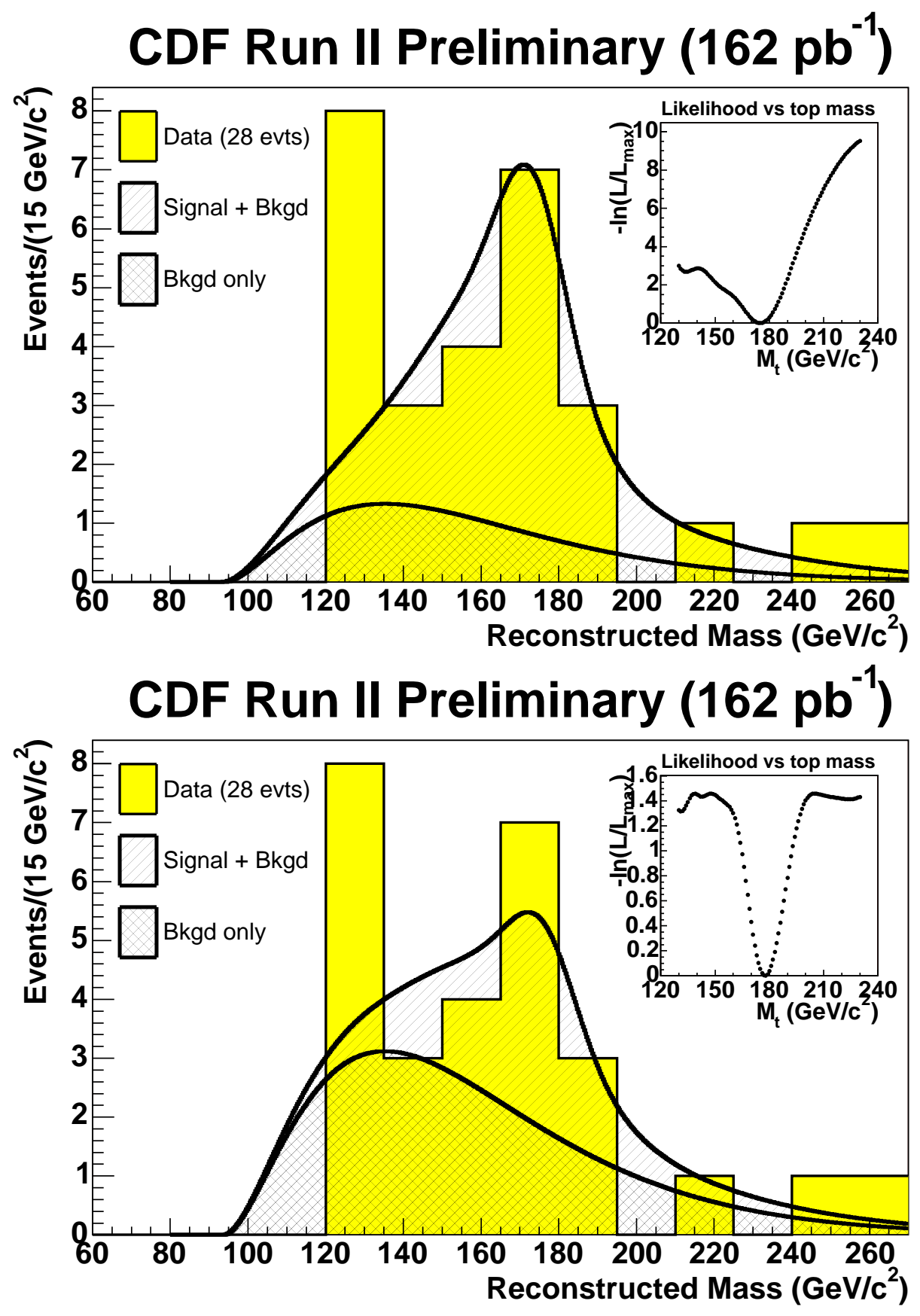

Figure 7.1: The reconstructed mass distribution plotted with the expected distributions from the Monte Carlo signal template at the fit mass and the background template for the $W+\geq 3.5$ jet sample. The background normalization is constrained in the upper plot and not constrained in the lower plot. In the lower plot, the points on the likelihood curve (inset) for which $139 \mathrm{GeV} / c^{2} \leq \mathrm{M}_{\text {top }} \leq 147 \mathrm{GeV} / c^{2}$ and $206 \mathrm{GeV} / c^{2} \leq \mathrm{M}_{\text {top }}$ are best fit with $f_{b g}>1$. 


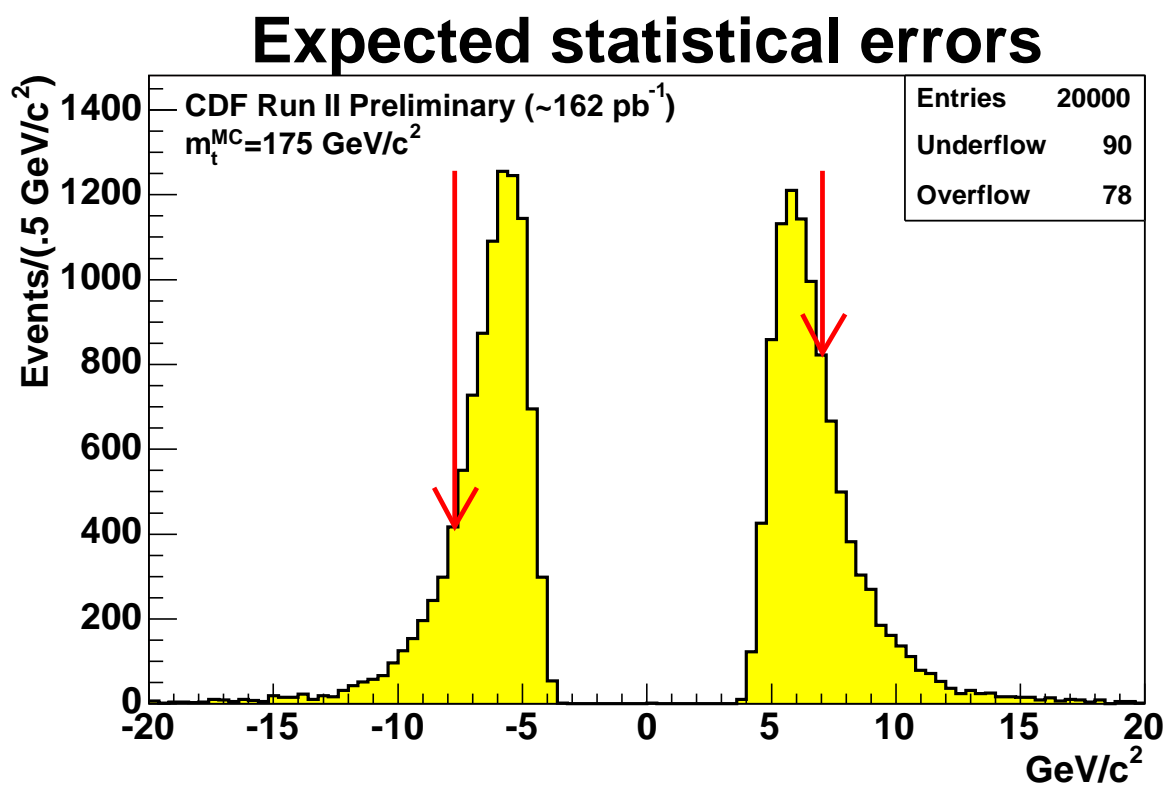

Figure 7.2: The positive and negative scaled statistical uncertainty distributions from 10,000 28-event pseudoexperiments for an input top mass of $175 \mathrm{GeV} / c^{2}$. The red arrows indicate the statistical uncertainties in the data.

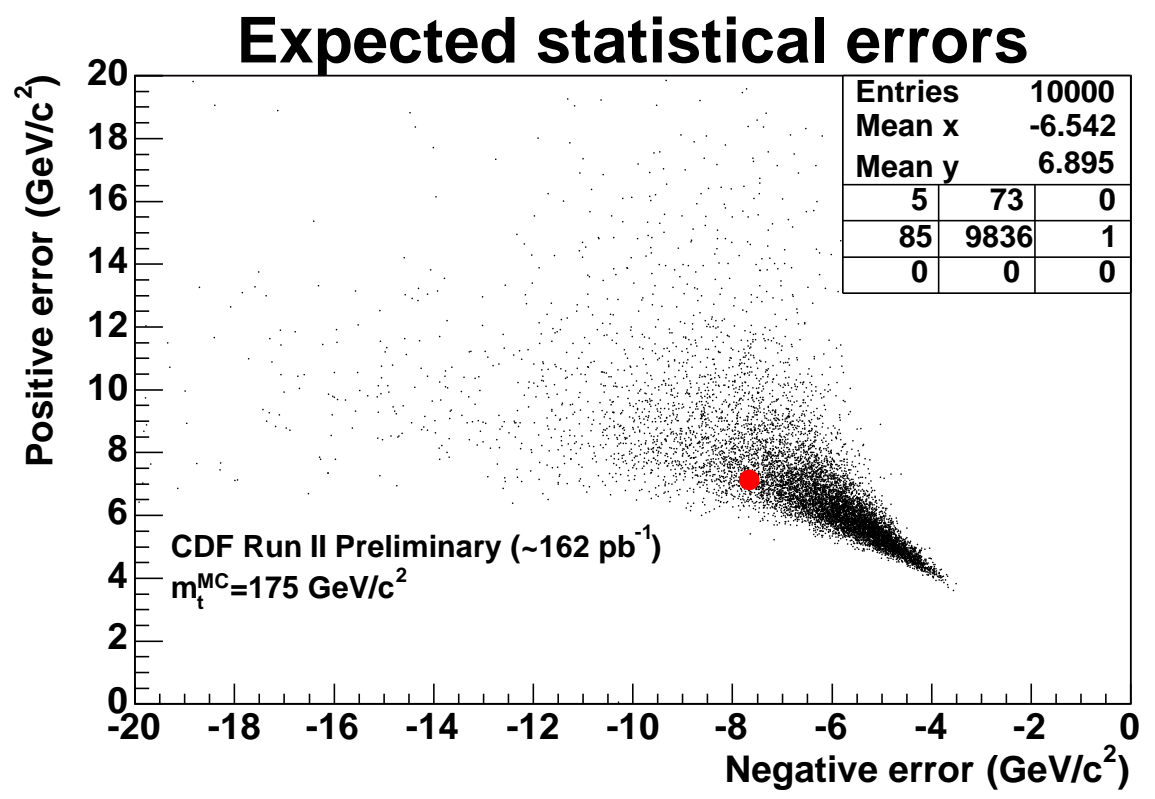

Figure 7.3: The positive vs negative scaled statistical uncertainty from 10,000 28-event pseudoexperiments for an input top mass of $175 \mathrm{GeV} / c^{2}$. The red point indicates the positive and negative statistical uncertainties in the data. 

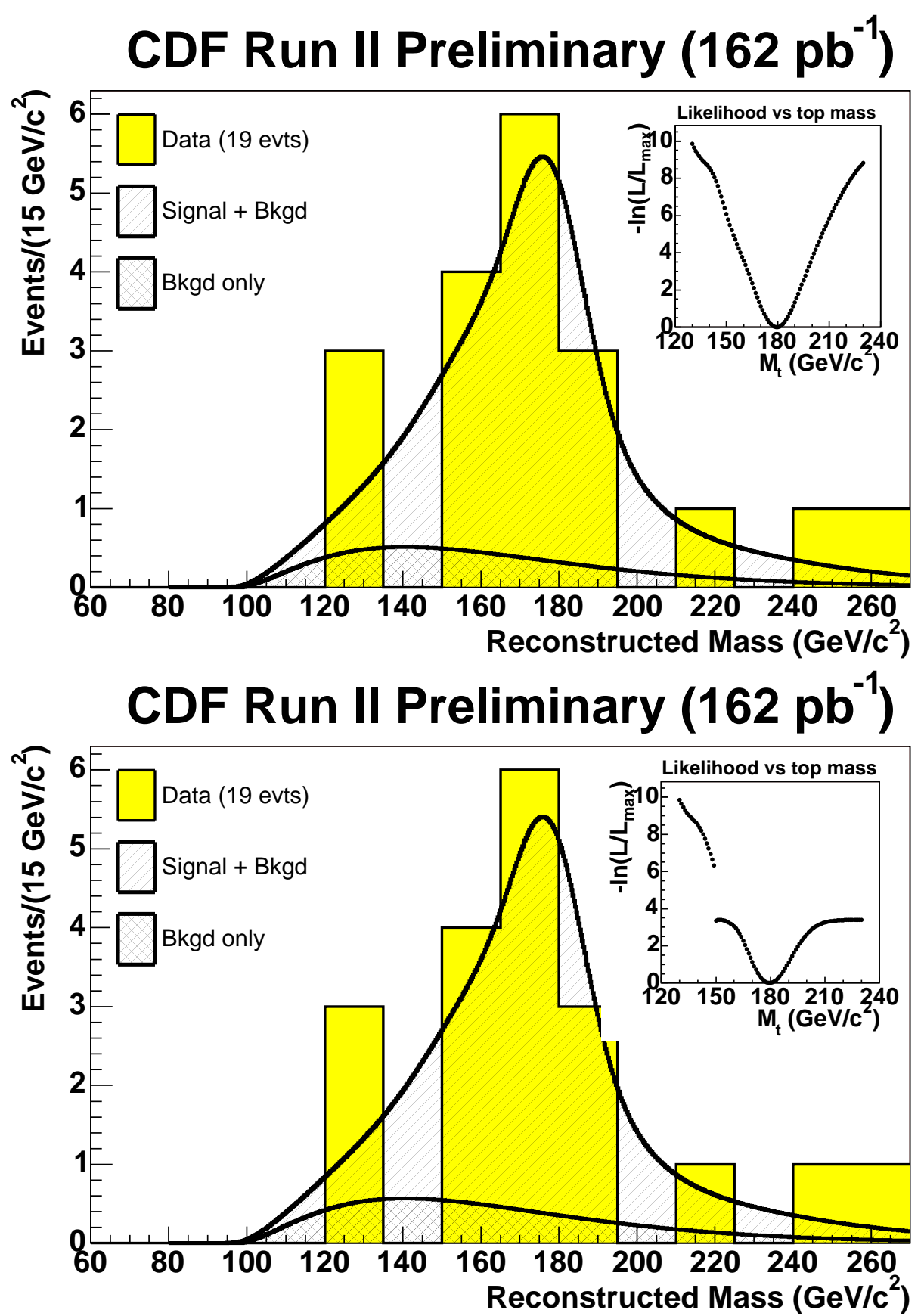

Figure 7.4: The reconstructed mass distribution plotted with the expected distributions from the Monte Carlo signal template at the fit mass and the background template for the $W+\geq 4$ jet sample. The background normalization is constrained in the upper plot and not constrained in the lower plot. In the lower plot, the points on the likelihood curve (inset) for which $150 \mathrm{GeV} / c^{2} \leq \mathrm{M}_{\text {top }} \leq 152 \mathrm{GeV} / c^{2}$ are best fit with $f_{b g}>1$. 


\section{Chapter 8}

\section{Systematic Uncertainties}

In the limit of perfect Monte Carlo generation and perfect detector simulation, this method would have no systematic errors. A more optimal event-by-event mass reconstruction would produce a measurement with better statistical resolution, but any shifts or effects in the reconstructed mass distribution would be, on average, the same in Monte Carlo and data, so that no systematic effects could be present. Of course, that condition does not obtain. But it is helpful to understand that all systematics are, at their root, due to differences between the data and our Monte Carlo description both of physical processes and of our detector.

We have considered the following sources of systematic uncertainty on the top mass: the jet energy scale/jet fragmentation, the jet resolution, the generated top $p_{T}$ distribution, our understanding of the reconstructed mass shape in the background events, the amount of initial and final state radiation, the parton distribution functions, and the SecVtx $b$-tagging scale factor.

Systematic uncertainties are estimated by looking at simulated Monte Carlo sam-

ples, in order to avoid biases due to the particular set of events in the data. For each source of systematic uncertainty, we vary quantities associated with the uncertainty source by $\pm 1 \sigma$, and produce new $175 \mathrm{GeV} / c^{2} t \bar{t}$ signal and background Monte Carlo templates by performing event selection and mass reconstruction on the modified samples. We draw events for pseudoexperiments from these new templates, but the signal and background p.d.f.'s are always unchanged. We then find the shift in the median fitted top mass for a large ensemble of pseudoexperiments. See Appendix A for details regarding pseudoexperiments, including a discussion of the uncertainties we quote for the systematic shifts. The 
uncertainty on a given systematic shift is added in quadrature to the shift to obtain the final systematic error.

Unless otherwise noted, all systematics described here have been estimated using 28 -event pseudoexperiments where the number of background events is generated with a Poisson mean of $7.0 \pm 0.8$.

\subsection{Jet Energy Scale and Jet Fragmentation}

There are significant uncertainties on many aspects of our measurement of jet energies. Some of these are in the form of uncertainties on our energy measurements themselves; some are uncertainties on our detector simulation, which is used to derive many corrections, and ultimately to extract the top mass; still others are best understood as uncertainties on jet production and fragmentation models used in the generators. In practice, we define systematics on the jet energies corresponding to the seven levels of generic jet corrections, described in section 5.1.2. The jet energy systematics, as well as the procedure to evaluate the resulting systematic uncertainty on the top mass measurement, are described in this section.

\subsubsection{Uncertainties on the jet energy}

The details of the jet corrections and the sources and amounts of systematic uncertainties are briefly described below:

$\eta$-Dependent Uncertainties The smoothing of the detector response as a function of $\eta$ is tested by photon-jet balancing in data and simulation. This uncertainty takes into account residual spline-interpolation effects, biases in the dijet balancing procedure, especially in the crack region, variation of plug calorimeter response with time, differences seen in the photon-jet balancing, and half the difference between the corrections for data and simulation. Table 8.1 summarizes the uncertainty that depends on $\eta$.

Central Calorimeter Stability A 1\% uncertainty for the stability of the central calorimeter response is assigned.

Raw Energy Scale Another systematic uncertainty is associated with the level 3 correction for the single-particle response of the central calorimeter. The difference between 
Table 8.1: The $\eta$-dependent systematic uncertainties on jet energies are shown for both data and simulation.

\begin{tabular}{|c|c|c|}
\hline$\eta$ range & data & simulation \\
\hline$|\eta|<0.2$ & $3 \%$ & $1 \%$ \\
$0.2<|\eta|<0.6$ & $0.5 \%$ & $1 \%$ \\
$0.6<|\eta|<1.0$ & $2 \%$ & $1 \%$ \\
$1.0<|\eta|<1.4$ & $4 \%$ & $7 \%$ \\
$1.4<|\eta|<2.0$ & $2 \%$ & $6 \%$ \\
$|\eta|>2$ & $7 \%$ & $7 \%$ \\
\hline
\end{tabular}

run I and run II energy scales is studied using photon-jet balancing and is measured to be $5 \%$, at least $2 \%$ of which comes from a difference in the CEM scale definition and additional material in the run II tracking volume, which results in a softer particle spectrum due to external bremsstrahlung and conversions. The remaining $3 \%$ difference is taken as a systematic uncertainty on the data jet energies.

A separate comparison of the photon-jet balancing in data and simulation finds a maximal difference of $5 \%$ between the two. Subtracting the data uncertainty of $3 \%$ in quadrature yields an independent uncertainty of $4 \%$ for the simulation energy scale.

Jet Fragmentation, Calorimeter Linearity, Underlying Event This systematic, associated with the level 5 and 6 generic corrections, determines uncertainties due to the modeling of jet showering and hadronization by varying relevant parameters in the generator.

Out-of-Cone Energy The uncertainty on the fraction of energy contained in the jet cone (also due to jet fragmentation modeling) is estimated in two parts, one between $R=$ 0.4 and $R=1.0$ and the other between $R=1.0$ and $R=\infty$.

\subsubsection{Uncertainties on the top mass due to jet energy systematics}

For each systematic uncertainty defined above, we apply a systematic shift to the jets in both the positive $(+1 \sigma)$ and negative $(-1 \sigma)$ directions. For each such shift, we reconstruct the mass in a given simulated sample using the shifted jets. Thus the mass fitter is applied $2 \times N_{\text {syst }}$ times to each event, where $N_{\text {syst }}=8$ is the number of systematic uncertainties due to the jet energy scale (see Table 8.2). 
Table 8.2: The systematic uncertainties on the top mass measurement are shown corresponding to each jet energy systematic described in section 8.1.1. Systematics are estimated both for the $W+\geq 3.5$-jet sample and the $W+\geq 4$-jet sample.

\begin{tabular}{|l|c||c|c|}
\hline \multicolumn{2}{|c||}{ Source of Corrections } & \multicolumn{2}{c|}{$\Delta \mathrm{M}_{\text {top }}\left(\mathrm{GeV} / c^{2}\right)$} \\
\hline Level & Description & $\geq 3.5$ jets & $\geq 4$ jets \\
\hline \hline 1 (sim) & $\eta$-Dependent Calibration $* *$ & $2.40 \pm 0.11$ & $2.53 \pm 0.11$ \\
1 (data) & $\eta$-Dependent Calibration $* *$ & $1.74 \pm 0.11$ & $1.89 \pm 0.11$ \\
2 (data) & Calorimeter Stability & $0.88 \pm 0.11$ & $1.11 \pm 0.11$ \\
\hline 3 (sim) & Raw Scale (central) $* *$ & $3.57 \pm 0.11$ & $3.60 \pm 0.11$ \\
3 (data) & Raw Scale (central) $* *$ & $2.72 \pm 0.11$ & $2.82 \pm 0.11$ \\
\hline \hline 5 & Absolute Scale & $2.20 \pm 0.11$ & $2.34 \pm 0.11$ \\
7 & Out-of-cone: up to cone 1.0 & $1.72 \pm 0.11$ & $1.79 \pm 0.11$ \\
& Out-of-cone: outside cone 1.0 & $1.47 \pm 0.12$ & $1.49 \pm 0.11$ \\
\hline \multicolumn{2}{|c|}{ Total } & $6.3 \pm 0.3$ & $6.6 \pm 0.3$ \\
\hline
\end{tabular}

For each shift (say, the raw energy scale systematic, $-1 \sigma$ ), signal and background reconstructed mass distributions are generated using all standard analysis cuts. A large number of pseudoexperiments are performed, drawing events from those shifted distributions. The number of events in each pseudoexperiment, and the background constraint, is the same as in the fit to the data events. Each systematic uncertainty is defined as half the difference between the medians of the results of the $+1 \sigma$ and the $-1 \sigma$ pseudoexperiments.

For the $\eta$-dependent and raw energy scale systematics, the uncertainties are different, and independent, for Monte Carlo and data jets. In order to estimate the uncertainty due to data jet systematics, we need to apply those data jet systematic shifts to Monte Carlo jets. We determine three jet corrections for each jet: the MC correction with no systematic applied; the data correction with the systematic applied; and the data correction with no systematic applied. We define the shift $\Delta$ as the difference between the latter two corrections. Then we apply the correction ( "corr" $+\Delta$ ), where "corr" is the first correction above, to the Monte Carlo jet. Each jet is corrected thusly, and the rest of the procedure is as above.

The out-of-cone and splash-out uncertainty need to be treated differently since they are defined only for jets corrected through level 7, and we use jets corrected through level 5 as input to the $t \bar{t}$-specific corrections. We take the absolute $p_{T}$ shift defined on the jets corrected through level 7 and add it to the jets corrected through level 5 . This procedure slightly overestimates the uncertainty, but the effect is small and does not affect 
Table 8.3: The top mass shift for various signal templates relative to a default are listed. Systematics are estimated both for the $W+\geq 3.5$-jet sample and the $W+\geq 4$-jet sample.

\begin{tabular}{|c||c||r|r|}
\hline \multicolumn{2}{|c|}{ Source } & \multicolumn{2}{c|}{$\Delta \mathrm{M}_{\text {top }}\left(\mathrm{GeV} / c^{2}\right)$} \\
\cline { 3 - 4 } \multicolumn{2}{|c||}{} & $\geq 3.5$ jets & $\geq 4$ jets \\
\hline \hline \multirow{2}{*}{ Gen } & Pythia - Herwig & $+0.3 \pm 0.3$ & $+0.2 \pm 0.3$ \\
\hline \hline \multirow{2}{*}{ PDF } & MRST72 - CTEQ5L (diff. group) & $0.0 \pm 0.3$ & $-0.2 \pm 0.3$ \\
\cline { 2 - 4 } & MRST75 - MRST72 $\left(\alpha_{s}\right.$ var. $)$ & $+0.2 \pm 0.3$ & $+0.6 \pm 0.3$ \\
\hline \hline \multirow{3}{*}{ ISR } & Tune B - Tune A & $+0.6 \pm 0.3$ & $+0.5 \pm 0.3$ \\
\cline { 2 - 4 } & Less ISR & $+0.3 \pm 0.3$ & $+0.3 \pm 0.3$ \\
\cline { 2 - 4 } & More ISR & $+0.3 \pm 0.3$ & $+0.6 \pm 0.3$ \\
\hline \hline \multirow{3}{*}{ FSR } & $\alpha_{s}$ var. & $+1.2 \pm 0.3$ & $+1.4 \pm 0.3$ \\
\cline { 2 - 4 } & NLO corr. off -on & $-1.4 \pm 0.3$ & $-1.5 \pm 0.3$ \\
\cline { 2 - 4 } & Less FSR & $+0.7 \pm 0.3$ & $+0.7 \pm 0.3$ \\
\cline { 2 - 4 } & More FSR & $+0.8 \pm 0.3$ & $+1.0 \pm 0.3$ \\
\hline \hline Spin & off - on & $+0.6 \pm 0.3$ & $+0.8 \pm 0.3$ \\
\hline
\end{tabular}

the total jet systematic uncertainties.

Table 8.2 lists the jet uncertainties obtained for the top mass measurement. The total systematic uncertainty in the top mass due to jet energy determination is $6.3 \pm 0.3 \mathrm{GeV} / c^{2}$ for the measurement using the $W+\geq 3.5$-jet sample. With the $W+\geq 4$-jet sample, the uncertainty is estimated to be $6.6 \pm 0.3 \mathrm{GeV} / c^{2}$, slightly larger than the uncertainty for the $\geq 3.5$-jet sample. As illustrated in Figure 8.1 (top), there seems to be a small correlation between the uncertainty and the sum of the leading four jet transverse energies, $\Sigma E_{T}^{4 j}$. The $\Sigma E_{T}^{4 j}$ of the $\geq 4$-jet sample is higher than that of the $\geq 3.5$-jet sample.

\subsection{Generators}

We estimate the systematic effect due to possible problems in the way our generator models its physical processes by comparing the Pythia and HERWIG generators. When the two corresponding $175 \mathrm{GeV} / c^{2} t \bar{t}$ samples are used to generate pseudoexperiments, the shift in top mass between them is found to be $0.3 \pm 0.3 \mathrm{GeV} / c^{2}$ (see Table 8.3). We declare this as the systematic uncertainty due to the generators. Note that this uncertainty may be contained by the jet fragmentation uncertainty (cf. level 5 and level 7 jet uncertainties). 

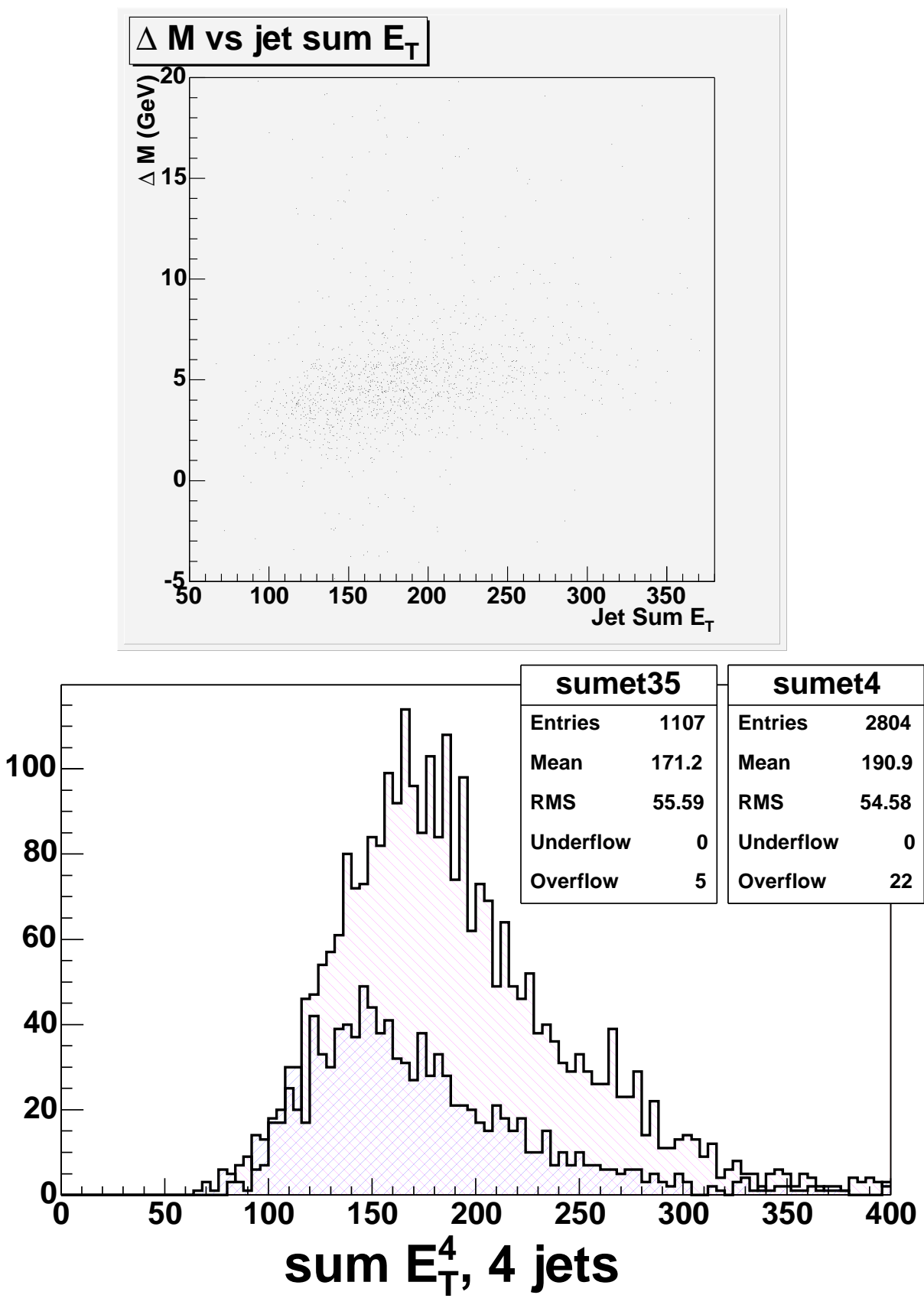

Figure 8.1: Upper plot: the uncertainty in top mass due to level 3 jet energy systematics as a function of the $\Sigma E_{T}$ of the leading 4 jets. Lower plot: the $\Sigma E_{T}$ distributions are shown for the $W+3.5$-jet sample and the $W+\geq 4$-jet sample. 


\subsection{Initial State and Final State Radiation}

The initial state radiation effect is studied using a special tuning of the Pythia parameters that reproduces well the underlying event, as well as with Pythia events with ISR enhanced and reduced to cover the present uncertainty in the rate of ISR. The results are summarized in Table 8.3. Since the median top mass from the default Pythia sample is not between the median top mass from the "less ISR" and the "more ISR" samples, we use the largest difference, $0.3 \pm 0.3 \mathrm{GeV} / c^{2}$, as the systematic.

The final state radiation effect is studied by varying the amount of final state radiation in the event to cover current uncertainties. The results are summarized in Table 8.3. Since the median top mass from the default Pythia sample is not between the median top mass from the "less FSR" and the "more FSR" samples, we use the largest difference, $0.8 \pm 0.3 \mathrm{GeV} / c^{2}$, as the systematic.

\subsection{Parton Distribution Functions}

The effect of PDFs is studied using the reweighting technique, in which we do not reconstruct samples independently generated and simulated using various PDFs. Instead, one single sample is used (the standard HERWIG $175 \mathrm{GeV} / c^{2}$ sample), and reconstructed mass templates for each PDF are generated by weighting the mass for each event by the probability for that event to proceed according to the given PDF (relative to the default PDF used to generate the sample).

We generate 46 reconstructed mass templates, where the PDFs used are

0 - CTEQ5L Our default PDF. The relative weight for every event is identically 1.

1 - MRST72 A leading-order PDF using more or less the same data as our default but fitted by a different group. No significant difference is expected relative to the default, but if a difference is found, it should be taken as a systematic.

2 - MRST75 Same as the previous one, but using a different value of $\alpha_{s}$, corresponding to $\Lambda_{Q C D}=300 \mathrm{MeV}$ vs $\Lambda_{Q C D}=228 \mathrm{MeV}$ for previous. The difference between these two PDFs is taken as a systematic.

3 - CTEQ6L More recent fit from CTEQ group, still leading order. 
4 - CTEQ6L1 Updated version of CTEQ6L.

5 - CTEQ6M This is the next-to-leading-order PDF from the CTEQ group. Using a leading-order matrix element, as the one found in HERWIG, in conjunction with this PDF will not give a correct description of event rates. However, we assume in the following that the relative behavior of various NLO PDFs is accurately modeled even though we use a LO matrix element. ${ }^{1}$

6-45 - CTEQ6M uncertainty PDFs These PDF sets encode the uncertainty in the CTEQ6M PDF. The possible variations are separated into contributions from 20 independent eigenvectors. Each eigenvector has a " $+1 \sigma$ " and a " $-1 \sigma$ " set, so for example sets 6 and 7 correspond to the $\pm 1 \sigma$ variations of eigenvector 1 , and sets 44 and 45 to $\pm 1 \sigma$ of eigenvector 20 .

Interesting quantities from the resulting 46 templates are shown in Figure 8.2. Each plot shows some information about the reweighted templates vs PDF number, and in each plot the central value NLO PDF (\#5) is highlighted in blue (large point), and a blue line is drawn through the 40 systematic PDFs at that value. The integral (top left) gives information about the change in the overall cross-section (folded in with our selection cuts). Again, our generator uses LO PDFs with a LO matrix element, so the comparison with NLO PDFs is not meaningful. But throughout this exercise, we assume that at least the differences between NLO PDFs can be trusted. The mean (top right), median (bottom left), and RMS (bottom right) of the templates track each other closely, but not exactly. A simple mechanism for change in the template, such as a linear slope in PDF probability vs reconstructed mass, is equivalent to rescaling the $\mathrm{x}$ axis, and would cause these three quantities to move together.

Note the $y$-axis scale in Figure 8.2. The differences are quite small. The largest effect in the CTEQ systematics is expected a priori for eigenvector \#15, corresponding to PDFs 34 and 35. This is the systematic dealing with the gluon distribution at high $x$. Indeed, the difference in the template medians is largest for this eigenvector, but it is only of the order of $0.1 \mathrm{GeV} / c^{2}$. Slightly larger differences are observed between PDF sets 0,1 , and 2 .

\footnotetext{
${ }^{1}$ This assumption breaks down at some point since, for example, the contribution from gluon-gluon fusion in NLO is 2-3 times as important as in LO. Thus contributions to the systematics due to uncertainty in the gluon distribution may be underestimated since we use a LO matrix element.
} 

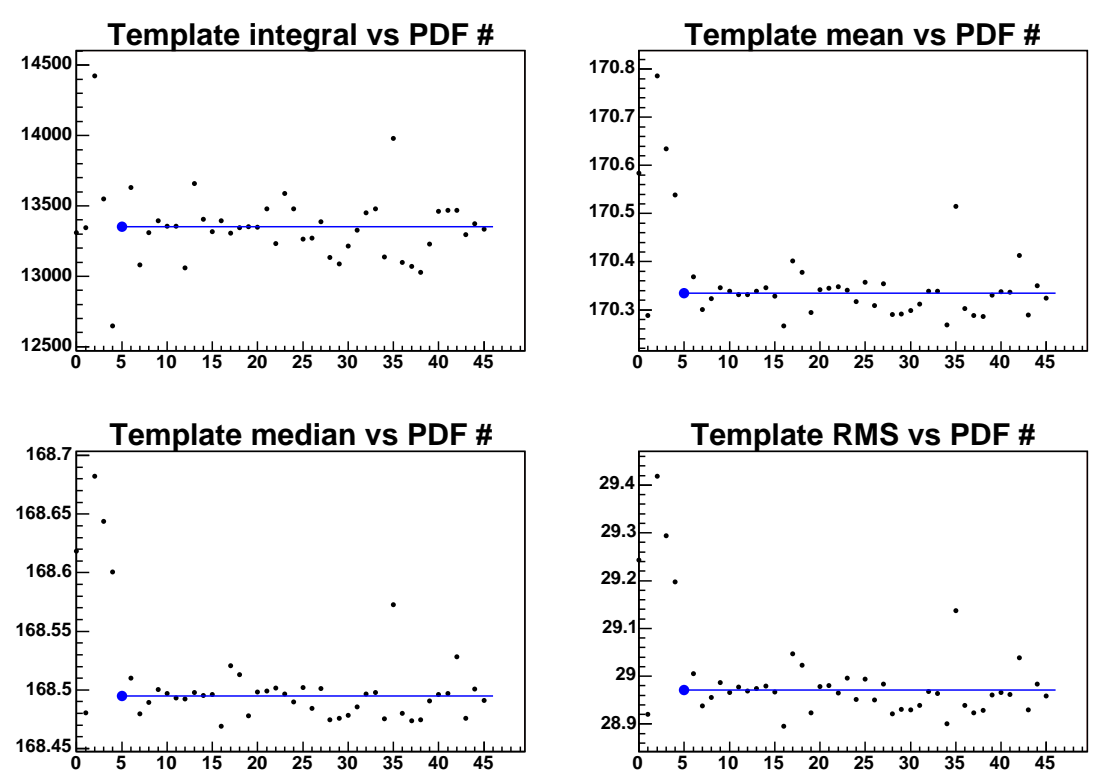

Figure 8.2: The integral (top left), mean (top right), median (bottom left), and RMS (bottom right) for each reweighted template are plotted vs PDF number. The central value NLO PDF (\#5) is highlighted in blue (large point), and a blue line is drawn through the 40 systematic PDFs at its value.

Next we perform pseudoexperiments using each reweighted template as the signal input, in order to estimate the systematics. The background input template is always our default. $^{2}$ Now, an important advantage of the reweighting technique is that it removes most of the difference between the templates that can be attributed to statistical fluctuations, so that we expect the largest contribution to the uncertainty on our systematic to come from the pseudoexperiment statistics. Accordingly, we run a large number of pseudoexperiments, 50,000, for each reweighted template.

Since the RMS for the distribution of fitted masses from the pseudoexperiments is typically $7.9 \mathrm{GeV} / c^{2}$, we expect an uncertainty on the mean of that distribution of $7.9 / \sqrt{50000}=0.035 \mathrm{GeV} / c^{2}$. Thus we expect an uncertainty on the difference between two medians of $\sqrt{2} * 0.035=0.050 \mathrm{GeV} / c^{2}$, and an uncertainty on half the difference of $0.050 / 2=0.025 \mathrm{GeV} / c^{2}$. The latter quantity is the systematic quoted for each eigenvector:

$$
\frac{\operatorname{Med}[+\sigma]-\operatorname{Med}[-\sigma]}{2} .
$$

\footnotetext{
${ }^{2} \mathrm{~A}$ more complete study would include the effects of the different PDFs on the background distributions as well.
} 

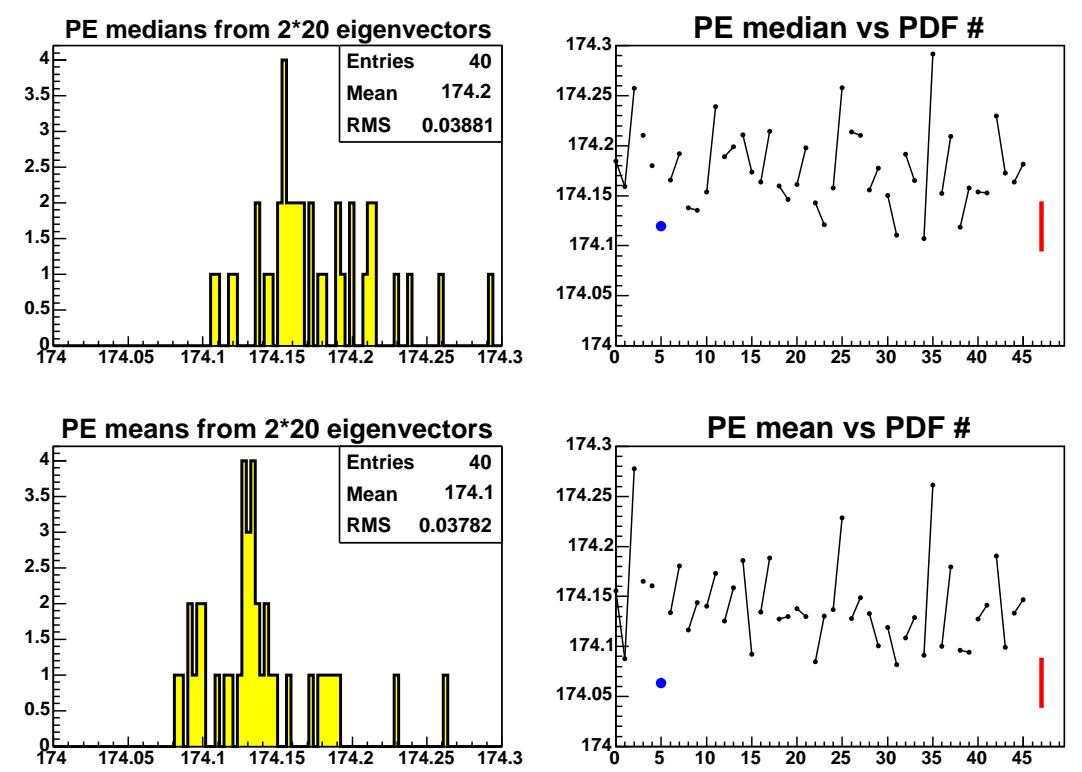

Figure 8.3: Results used for PDF systematic, from 50,000 pseudoexperiments for each reweighted template. The top left plot histograms the median mass from pseudoexperiments for the 40 CTEQ systematic PDFs. The top right plot shows the median mass from pseudoexperiments vs PDF number for all 46 PDFs. The bottom plots use the mean mass rather than the median.

So even if we observe 0 difference between the $+\sigma$ and $-\sigma$ templates for each eigenvector, our prescription of adding the uncertainty in quadrature with the observed shift would yield $0 \pm 0.025 \mathrm{GeV} / c^{2}$ for each of 20 eigenvectors, or a $0.112 \mathrm{GeV} / c^{2}$ total PDF eigenvector uncertainty. Of course, even if there were no real differences, we would still expect typical differences of the same order due to the statistical fluctuations, so that the expected uncertainty for each eigenvector would be $0.025 \pm 0.025 \mathrm{GeV} / c^{2}$, for a total uncertainty of $0.158 \mathrm{GeV} / c^{2}$.

The medians and means of the pseudoexperiment masses for each PDF are shown in Figure 8.3. The left plots show the actual distribution of medians (top) and means (bottom) from the 50,000 pseudoexperiments for the 40 CTEQ systematic PDFs. The RMS on the means and medians is $\sim 0.038 \pm 0.004 \mathrm{GeV} / c^{2}$, not much larger than the $0.035 \mathrm{GeV} / c^{2}$ we expect, so whatever real effects there are in the CTEQ systematics are small.

On the right in Figure 8.3 are the medians and means of the pseudoexperiment masses vs PDF number. The default NLO PDF (\#5) is highlighted in blue (large point), 
and the pairs of $\pm \sigma$ PDFs for each eigenvector are joined by thin black lines. (The blue point may seem "far" from the others, but it is less than $2 \sigma$ away from the mean of the remaining PDFs.) At the far right is a red line indicating the typical difference expected from statistics alone $\left(0.050 \mathrm{GeV} / c^{2}\right)$. Adding half the difference between each pair in quadrature gives a central shift in the top mass due to the CTEQ PDF systematics of $0.132 \mathrm{GeV} / c^{2}$. Since the systematic on each eigenvector has an uncertainty of $0.025 \mathrm{GeV} / c^{2}$, we have a total systematic on the top mass due to the CTEQ PDF systematics of $\sqrt{0.132^{2}+20 \times 0.025^{2}}=0.173 \mathrm{GeV} / c^{2}$. As expected, the largest contribution of $\sim 0.14 \mathrm{GeV} / c^{2}$ comes from eigenvector \#15. ${ }^{3}$

Finally we estimate the remaining PDF uncertainties: the pseudoexperiments using PDF numbers 1 and 0 give a difference of $-0.026 \pm 0.050 \mathrm{GeV} / c^{2}$, while PDF numbers 2 and 1 have a difference of $0.099 \pm 0.050 \mathrm{GeV} / c^{2}$. Thus we take systematics due to PDF group and PDF $\alpha_{s}$ of $0.056 \mathrm{GeV} / c^{2}$ and $0.111 \mathrm{GeV} / c^{2}$, respectively. When fully simulated independent samples are used to determine these shifts, the results are consistent, though with much larger uncertainties, as given in Table 8.3.

Adding the three sources of PDF systematics in quadrature gives a total PDF uncertainty of $0.213 \mathrm{GeV} / c^{2}$. This is a large reduction from the previous (statistics-dominated) PDF uncertainty of $1.5 \mathrm{GeV} / c^{2}$.

\subsection{Jet Resolution}

The simulation does not model well the jet resolutions. As illustrated in $\gamma$-jet balance studies (see Table 8.4), the simulation underestimates the resolution by about $15 \%$ on average in this data sample. We estimate a possible bias in the top mass measurement by smearing the MC jets in the pseudoexperiment samples by an extra $15 \%$. (Thus jets with $20 \%$ energy resolution will have $\sqrt{(20 \%)^{2}+(15 \%)^{2}}=25 \%$ resolution after the smearing.) See Figure 8.4 for plots of the smearing effect and the change in the reconstructed mass template. Using the full machinery of pseudoexperiments, the top mass shift due to the extra smearing is found to be $-0.4 \pm 0.1 \mathrm{GeV} / c^{2}$. We take this as an uncertainty due to the

\footnotetext{
${ }^{3}$ It has been suggested that we double the effect of eigenvector \#15 in our systematic to account for the difference in the contribution of $g g \rightarrow t \bar{t}$ to the total $t \bar{t}$ cross-section at LO and NLO. However, it has also been pointed out that the CTEQ PDF systematics correspond not to $\pm 1 \sigma$, but to $\sim 90 \%$ confidence. Since we treat the resulting uncertainties on the top mass as $\pm 1 \sigma$ uncertainties, these two concerns at least partially cancel.
} 
Table 8.4: The mean and sigma of Gaussian fits to $\left(E_{T}^{j e t}-E_{T}^{\gamma}\right) / E_{T}^{j e t}$ distributions in the photon-jet sample. Jets are reconstructed using a cone of 0.4 .

\begin{tabular}{|c|c|c|c|}
\hline Jet corrections & & Data & MC \\
\hline none & Mean & $-0.385 \pm 0.002$ & $-0.299 \pm 0.003$ \\
& Sigma & $-0.201 \pm 0.001$ & $+0.169 \pm 0.002$ \\
\hline Level 7 & Mean & $+0.002 \pm 0.002$ & $+0.053 \pm 0.003$ \\
& Sigma & $+0.241 \pm 0.001$ & $+0.214 \pm 0.002$ \\
\hline
\end{tabular}

jet energy resolution. This uncertainty was estimated using 25-event pseudoexperiments corresponding to the $108 \mathrm{pb}^{-1}$ analysis.

We also checked the effects of the jet resolution in the fitting procedure by scaling jet resolutions $\left(\sigma_{j e t}\right.$ in Equation 5.2.1) by factors between 0.5 and 2. The top mass shift between the scale factor of 0.5 and that of 2 in the fitting is found to be $0.4 \pm 0.1 \mathrm{GeV} / c^{2}$.

Finally, we determined the jet resolutions using the RMS of the jet response after the top-specific corrections, instead of using the symmetric 68\% CL (our default), and used them in the mass fitting. A mass shift of $0.4 \pm 0.1 \mathrm{GeV} / c^{2}$ was observed in pseudoexperiments. Both of these checks are consistent with the quoted systematic.

\subsection{Top $p_{T}$ Distribution}

As described in section 5.1.3, the top-specific corrections depend on the $p_{T}$ spectrum of jets, thus the $p_{T}$ spectrum of top. It is possible that the Herwig and Pythia modeling of the top $p_{T}$ distribution is incorrect. We studied this possibility by constraining the top $p_{T}$ distribution using the CDF run I measurement [21]. The variation in the hadronic top mass (the invariant mass of the top quark that decays to two light quarks and one $b$ quark) is measured to be $0.4 \pm 0.4 \mathrm{GeV} / c^{2}$. Although this is statistically insignificant, we take it as a systematic on the MC modeling of the top $p_{T}$ distribution. Note that this systematic is estimated without the full machinery of pseudoexperiments.

\subsection{Background Shape}

The uncertainty due to the background reconstructed mass shape comes in two parts. The first is due to variations in the relative normalizations of the individual back- 

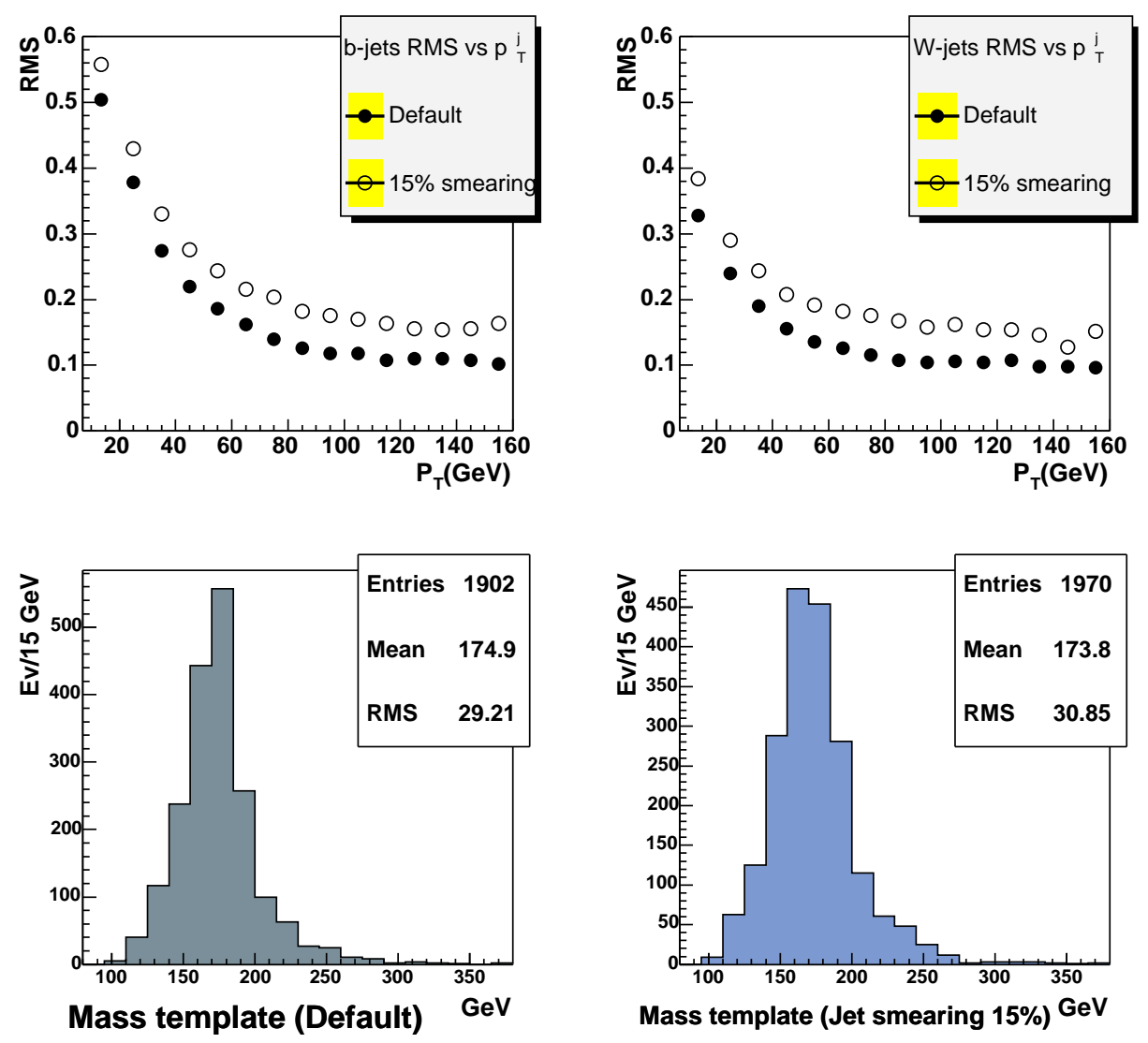

Figure 8.4: Upper plot: jet response as a function of $p_{T}^{\text {jet }}$ for $b$ jets and $W$ jets after generic corrections applied, for the default jets (full circles) and the jets with extra $15 \%$ smearing (open circles). Lower plot: the reconstructed mass distributions with the default simulation (left) and the simulation with extra $15 \%$ smearing on jet energies (right). 
Table 8.5: Top mass differences when the background shape is varied, but the same normalization is used. We vary the shape by using each background template separately in the fit, rather than combining them.

\begin{tabular}{|c|c|c|}
\hline \multirow{2}{*}{ Background Shape } & \multicolumn{2}{|c|}{$\Delta \mathrm{M}_{\text {top }}\left(\mathrm{GeV} / \mathrm{c}^{2}\right)$} \\
\cline { 2 - 3 } & $\geq 3.5$ jets & $\geq 4$ jets \\
\hline Combined & 0 (default) & 0 (default) \\
$W+$ jets & $+0.2 \pm 0.1$ & $-0.64 \pm 0.12$ \\
$W b \bar{b}$ & $-0.6 \pm 0.1$ & $-0.98 \pm 0.12$ \\
Single top (s channel) & $+0.1 \pm 0.1$ & $-0.51 \pm 0.12$ \\
Single top (t channel) & $+0.4 \pm 0.1$ & $-0.79 \pm 0.11$ \\
\hline Total & $0.5 \pm 0.1$ & $0.5 \pm 0.1$ \\
\hline
\end{tabular}

ground processes, since in our method the overall background normalization is allowed to float, but the relative normalizations are fixed. The second part is due to variations in the $Q^{2}$ used for the background event generation.

The relative normalization uncertainty is studied by using each of the $W b \bar{b}, W+$ jets (mistags), and single top mass templates (see Figure 6.5) as the background template in pseudoexperiments, rather than combining them as for the default measurement. The 3.5-jet and 4-jet templates for each process are combined in the expected fractions. The shifts in the top mass are summarized in Table 8.5. We assign half of the largest difference, $0.5 \pm 0.1 \mathrm{GeV} / c^{2}$, to be the uncertainty due to the background shape. This is a conservative estimate that also covers various possible problems with the background modeling.

The uncertainty due to the $Q^{2}$ used in the background simulation is estimated using $W b \bar{b}+1 \mathrm{p}$ samples generated using four different $Q^{2}$ values. The samples and the resulting top mass shifts are given in Table 8.6. We assign half of the largest difference, $0.5 \pm 0.3 \mathrm{GeV} / c^{2}$, as the systematic uncertainty.

\subsection{SecVtx $b$ Tagging}

We use the run I estimate of the uncertainty due to the SecVtx $b$-tagging scale factor energy dependence: $0.1 \mathrm{GeV} / c^{2}$. We exclude that part of the run I $b$-tagging uncertainty due to SLT tags.

In the default measurement, we do not correct the ratio of single- to double-tagged events in our templates for the data/MC tagging efficiency scale factor (see footnote 9 in 
Table 8.6: Median top masses from pseudoexperiments when the background shape is taken from $W b \bar{b}+1 \mathrm{p}$ samples generated using different $Q^{2}$.

\begin{tabular}{|c|c|c|}
\hline \multirow{2}{*}{$Q^{2}$} & \multicolumn{2}{|c|}{$\mathrm{M}_{\text {top }}\left(\mathrm{GeV} / \mathrm{c}^{2}\right)$} \\
\cline { 2 - 3 } & $\geq 3.5$ jets & $\geq 4$ jets \\
\hline$\left(2 M_{W}\right)^{2}$ & $174.4 \pm 0.2$ & $174.75 \pm 0.25$ \\
$M_{W}^{2}$ & $173.8 \pm 0.2$ & $174.35 \pm 0.27$ \\
$\left(M_{W} / 2\right)^{2}$ & $173.7 \pm 0.2$ & $173.72 \pm 0.25$ \\
$M_{W}^{2}+\left(p_{T}^{W}\right)^{2}$ & $174.7 \pm 0.2$ & $174.61 \pm 0.26$ \\
\hline$\Delta \mathrm{M}_{\text {top }}$ & $0.5 \pm 0.3$ & $0.5 \pm 0.3$ \\
\hline
\end{tabular}

chapter 5). For now, we check the effect of this omission in the following ways. First, we fit the data using corrected signal templates. There is no change from our default measurement or errors, at least at the quoted precision $\left(0.1 \mathrm{GeV} / c^{2}\right)$. Second, we perform pseudoexperiments in which we fit generated events using both the default and the corrected signal templates. The mean pseudoexperiment-by-pseudoexperiment shift between the two fits is $\sim 0.1 \mathrm{GeV} / c^{2}$, which is roughly the same size as our quoted $b$-tagging systematic, and negligible compared to other systematics.

\subsection{Additional MC Modeling Checks}

Another set of the top-specific corrections are extracted using the Pythia generator. The difference in the hadronic top mass between Herwig- and Pythia-based top-specific corrections is measured to be $0.1 \pm 0.1 \mathrm{GeV} / c^{2}$. When the full machinery of the pseudoexperiments is used, the difference becomes $0.5 \pm 0.1 \mathrm{GeV} / c^{2}$. The above checks are correlated with uncertainties due to initial state radiation and generators; we don't assign a separate systematic for these differences.

\subsection{Summary of Systematic Uncertainties}

The systematic uncertainties are summarized in Table 8.7. The total systematic uncertainty is estimated to be $6.5 \mathrm{GeV} / c^{2}$. 
Table 8.7: Summary of systematic uncertainties in the $W+\geq 3.5$ jets and $W+\geq 4$ jets analyses. The numbers in parentheses are taken from the $W+\geq 3.5$ jets analysis.

\begin{tabular}{|c|c|c|}
\hline \multirow{2}{*}{ Source of Systematics } & \multicolumn{2}{|c|}{$\Delta \mathrm{M}_{\text {top }}\left(\mathrm{GeV} / \mathrm{c}^{2}\right)$} \\
\cline { 2 - 3 } & $\geq 3.5$ jets & $\geq 4$ jets \\
\hline Jet Energy & 6.3 & 6.6 \\
ISR & 0.4 & 0.6 \\
FSR & 0.9 & 1.0 \\
PDFs & 0.2 & $(0.2)$ \\
Generators & 0.4 & 0.4 \\
Background Shape & 0.8 & 0.8 \\
Other MC modeling (Jet Resolution, $\left.p_{T}^{\text {top }}\right)$ & 0.7 & $(0.7)$ \\
$b$ tagging & 0.1 & $(0.1)$ \\
\hline Total & 6.5 & 6.8 \\
\hline
\end{tabular}




\section{Chapter 9}

\section{Other Checks}

This chapter contains various checks and studies performed with the aim of validating our understanding of the data, including the goodness of our Monte Carlo modeling. Most of the studies were performed with a subset of the data corresponding to $108 \mathrm{pb}^{-1}$. Any results using the full $163 \mathrm{pb}^{-1}$ will be noted.

\subsection{Subdivision of Top Mass Sample}

The statistical sensitivity of this analysis can be improved in principle by dividing the top mass sample into subsamples with different signal and background mass shape and background event rate. This strategy was employed for the run I analysis where four subsamples were used based on the $b$-tagging information [1].

We try two possibilities: first, we treat separately the single- and double-tag events, and secondly the 3.5- and 4-jet events. These results are only treated as crosschecks so far, but may be used to extract the final result in future versions of the analysis.

We derive a background normalization estimate assuming the same $\chi^{2}$ cut efficiency for double-tag events as for $\geq 1$-tag events (see Table 6.2). A total of $0.36 \pm 0.07$ double-tag background events is expected. We subtract this number from the $\geq 1$-tag expected background and get $6.6 \pm 0.8$ expected single-tag background events. The expected background for 3.5- and 4-jet events separately was given in Table 6.2. For each of these subsamples, the signal and background templates are extracted separately and a likelihood function is defined as in chapter 6 . The likelihoods of the associated subsamples are multiplied to get a combined likelihood. 
Table 9.1: $68 \%$ C.L. of expected statistical uncertainty from background-constrained fit when various subdivisions of the event sample are employed.

\begin{tabular}{|l|c|}
\hline Fitting procedure & Expected Uncert. $\left(\mathrm{GeV} / c^{2}\right)$ \\
\hline Default & $6.46 \pm 0.14$ \\
1/2-tag separate & $6.33 \pm 0.13$ \\
3.5/4-jets separate & $6.37 \pm 0.13$ \\
\hline
\end{tabular}

Table 9.2: Constrained fit results on data events for separate fit of 1/2-tag events and $3.5 / 4$-jet events

\begin{tabular}{|l|c|}
\hline Fitting procedure & Result $\left(\mathrm{GeV} / c^{2}\right)$ \\
\hline Default & $174.9_{-7.2}^{+6.7}$ \\
1/2-tag separate & $174.4_{-7.3}^{+6.7}$ \\
3.5/4-jets separate & $177.5_{-6.6}^{+6.5}$ \\
\hline
\end{tabular}

To test the fitting procedures, pseudo-experiments are generated. The same ratio of 1/2-tags events and 3.5/4-jets events used to create the default templates have been used as input to the pseudoexperiments in order to make a fair comparison. The $68 \%$ C.L. of the expected uncertainties (without any scaling of the errors) are shown in Table 9.1. The expected improvement is of the order of a few percent.

The results of these fits on the data are shown in Table 9.2. The $1 / 2$-tag result is understandably very close to the default one, since only two double-tag events are seen in the data. The 3.5/4-jets separation gives a central value slightly larger with significantly smaller uncertainties, probably since the 3.5- and 4-jet events are so differently distributed in data, and the 4-jet events are given more weight in the fit since they have sharper mass templates. The mass distributions fitted individually for the single-tag, double-tag, 3.5and 4-jet subsamples are shown in Figure 9.1.

\subsection{Alternate Top-Specific Corrections}

We checked the stability of the mass extraction by using the alternate top-specific corrections available at CDF. The differences between these two corrections are, briefly, that the alternate corrections:

- use only isolated jets, and for $b$ jets, use dilepton events 

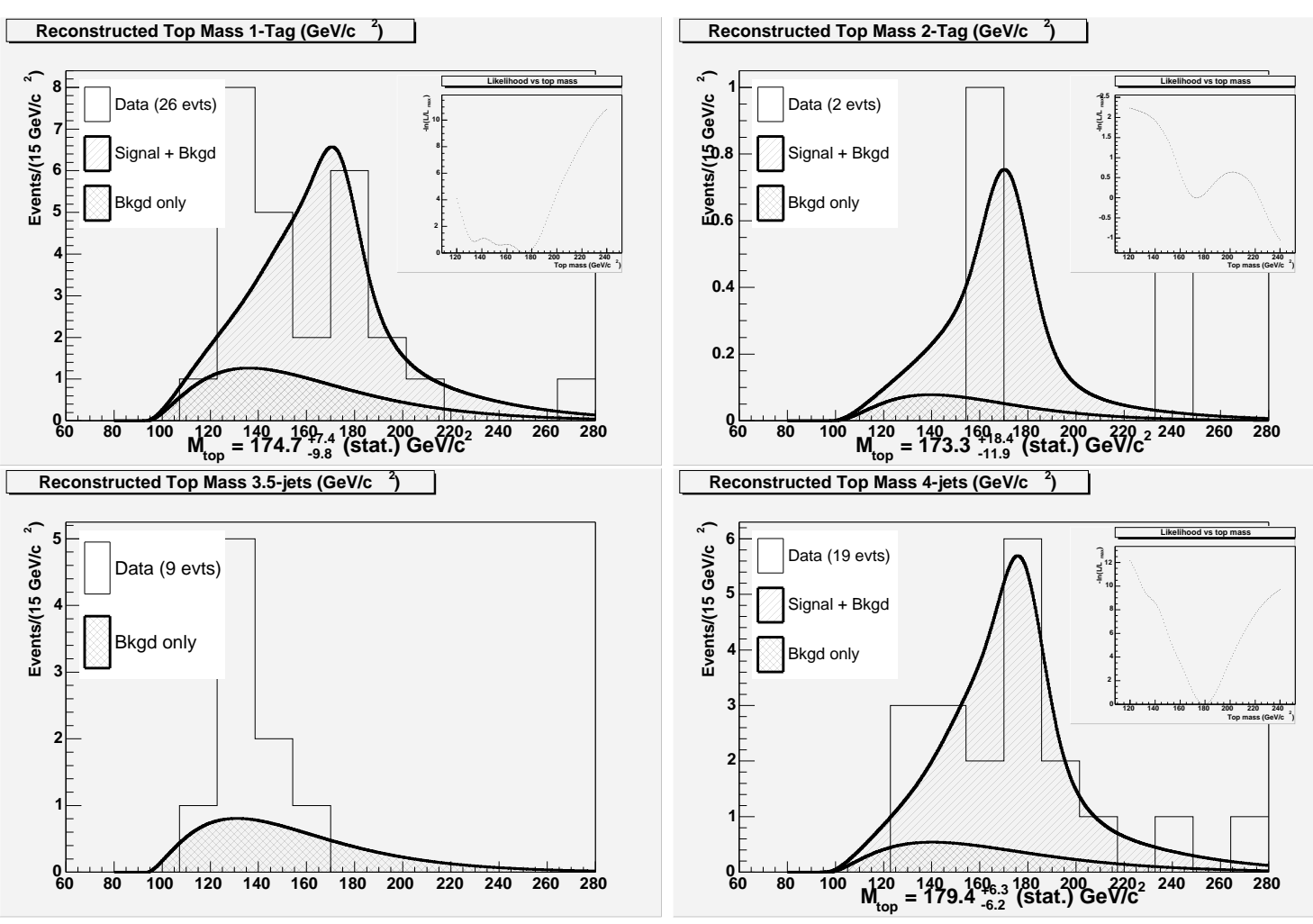

Reconstructed Top Mass 4-jets (GeV/c ${ }^{2}$ )

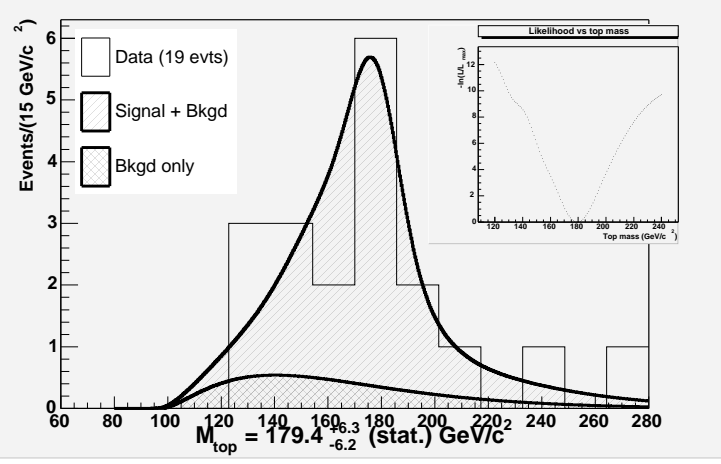

Figure 9.1: Fitted data mass distribution for single-tag events (top left), double-tag events (top right), and 4-jet events (bottom right). The fit does not converge for the 3.5-jet data distribution (bottom left); for 3.5-jet events, the data mass distribution is shown along with the background shape using the central value for the background normalization. 

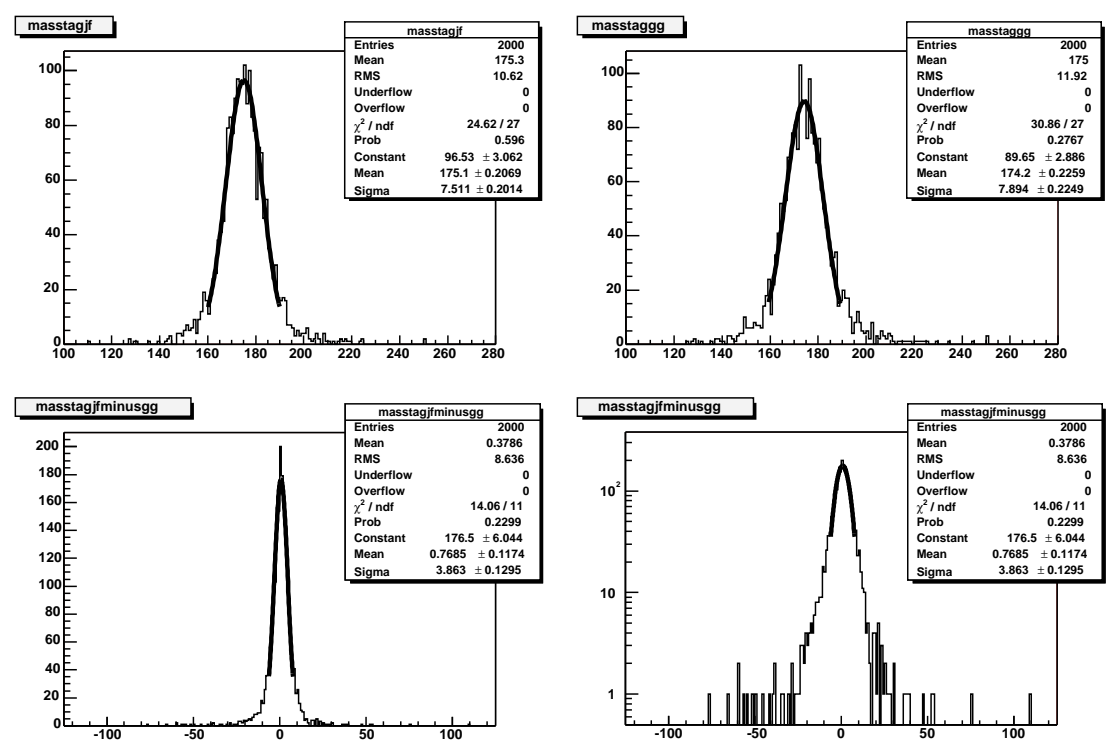

Figure 9.2: Top plots: the extracted mass distributions from 2000 pseudoexperiments using this analysis's top-specific corrections (top left) and the alternate corrections (top right). Bottom plots: top mass difference distribution from 2000 pseudoexperiments on a linear scale (bottom left) and a logarithmic scale (bottom right).

- are not $\eta$ dependent corrections

- take jet resolutions from the RMS of the response functions

- take jet corrections from the mean of the response functions

A complete analysis is done with the alternate top-specific corrections using pseudoexperiments, including remaking the MC templates and p.d.f.'s. To observe the expected differences between the two sets of corrections for a given set of events, we need to know the pseudoexperiment-by-pseudoexperiment difference in $\mathrm{M}_{\text {top }}$. We select a sample of events for each pseudoexperiment, two top-specific corrections (default and alternate) are applied to the sample, and the two corresponding fit top masses are extracted. The fitted mass distributions from 2000 pseudoexperiments are shown in Figure 9.2 for the default (top left) and alternate (top right) top-specific corrections. This figure also shows the fit mass difference between the two corrections from 2000 pseudoexperiments: the bottom left plot in the linear scale and the bottom right plot in the logarithmic scale. The average and width of the mass difference distribution are $0.8 \mathrm{GeV} / c^{2}$ and $3.9 \mathrm{GeV} / c^{2}$, respectively. 
A complete analysis is also done with the alternate top-specific corrections for the data. Figure 9.3 (top) shows the reconstructed mass difference for each event. The fitted top mass is found to be $2.2 \mathrm{GeV} / c^{2}$ lower than the default measurement, which is consistent with what we expect based on the pseudoexperiment studies.

\subsection{W Mass in the Hadronic Decay Mode}

An important intermediate handle we have in any $t \bar{t}$ event is the well known $W$ mass. If we pick the right jet-parton assignment, and the jets are corrected properly, we expect to find the invariant mass of the two $W$ daughter jets equal to the $W$ mass. Figure 9.4 (center) shows the invariant mass distributions of $W$ daughter jets where the jets are assigned by the mass fitter and the jet energies include the top-specific corrections but not the $W$ mass constraint. The agreement between the data and the signal + background combined shape from simulation is reasonable, but the statistics are poor. We increased statistics by removing the $b$-tagging requirement. The agreement is good and the results are shown in Figure 9.4 (right). We also show the distributions for double-tagged events, where the statistics are even more limited (Figure 9.4 left). For the comparison between data and Monte Carlo, we use a combination of signal and background MC in the ratios $95 / 5,75 / 25$, and 25/75 for two tag, at least one tag, and all events, respectively. For all three selections, a KS test implies consistent distributions.

\subsection{Lepton $\eta$ Dependence}

In this analysis we assumed that the lepton trigger efficiencies were flat in $\eta$ and the total integrated luminosity for CMX muons is the same as that of central electrons and CMUP muons. The latter, at least, is not true (see section 4.3). We compared the reconstructed mass distributions between the electron sample and the muon sample, and between the CMUP sample and the CMX sample. As illustrated in Figure 9.5, the average of the mass templates varies by $1 \sim 2 \mathrm{GeV} / c^{2}$ in the most extreme case. If there were a bias in the top mass shape as a function of $\eta$ or between electrons and muons, we would have a bias of a small fraction of $1 \mathrm{GeV} / c^{2}$ in the measurement. 

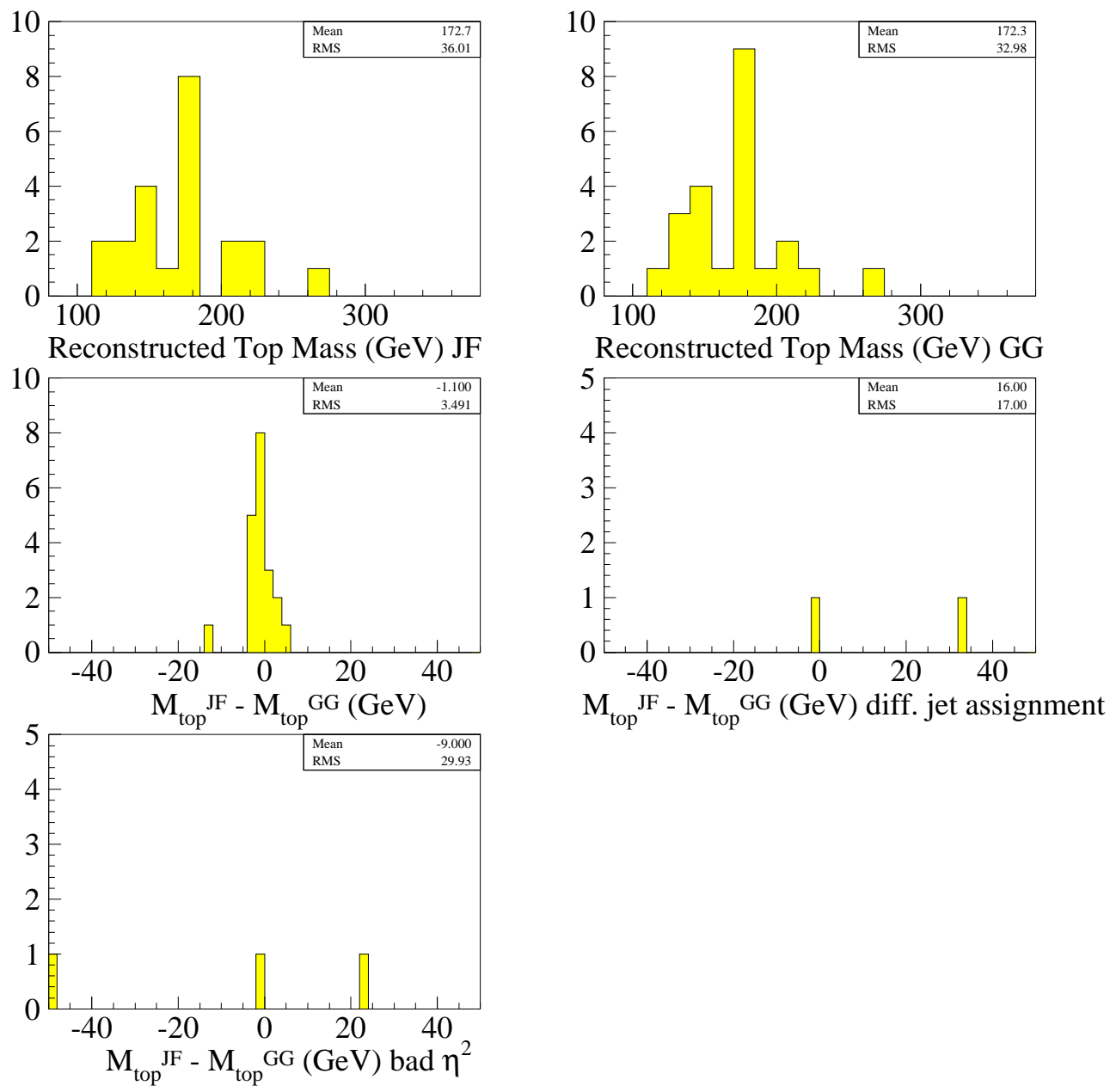

Figure 9.3: Reconstructed mass distributions for $108 \mathrm{pb}^{-1}$ with the default top-specific corrections (top left) and the alternate corrections (top right). Event-by-event mass differences between these two corrections for events with $\chi_{\min }^{2}<10$ and the same jet assignment (middle right), events with different jet assignments (middle right), and events with $\chi_{\min }^{2}>10$. 

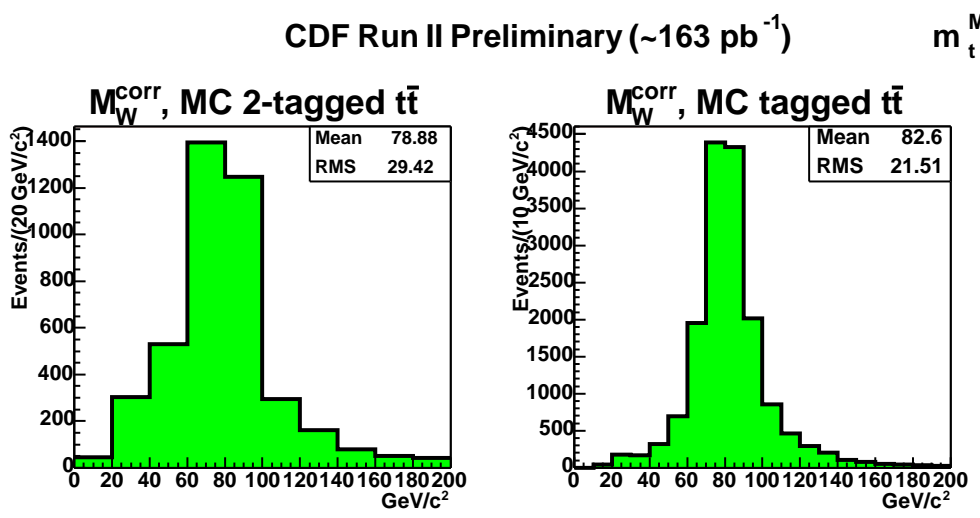

$m_{t}^{M C}=175 \mathrm{GeV} / \mathrm{c}^{2}$
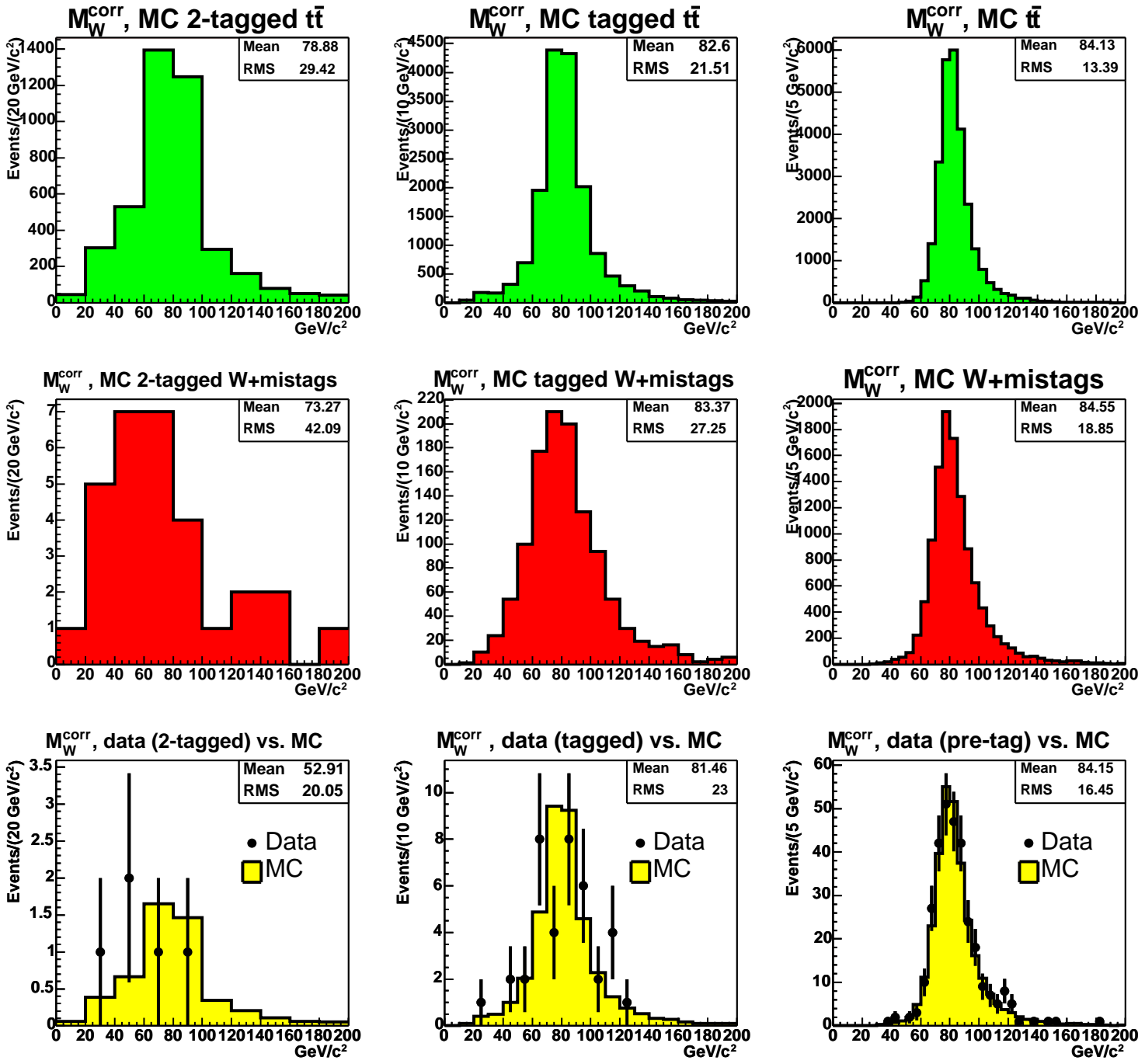

Figure 9.4: The invariant mass distributions of $W \rightarrow q \bar{q}^{\prime}$ jets where the jet assignment is determined by the top mass fitter and jet energies include top-specific corrections without the $M_{W}$ constraint. The left plots are for events with two $b$ tags, center plots show events with at least one $b$ tag, and the right plots do not require any $b$ tag in event selection or when choosing the jet assignment. Top, middle and bottom plots present the top signal, background $(W+$ jets $)$, and data plotted against Monte Carlo. We use a combination of signal and background in the ratios $95 / 5,75 / 25$, and 25/75 for left, middle, and right, respectively. 

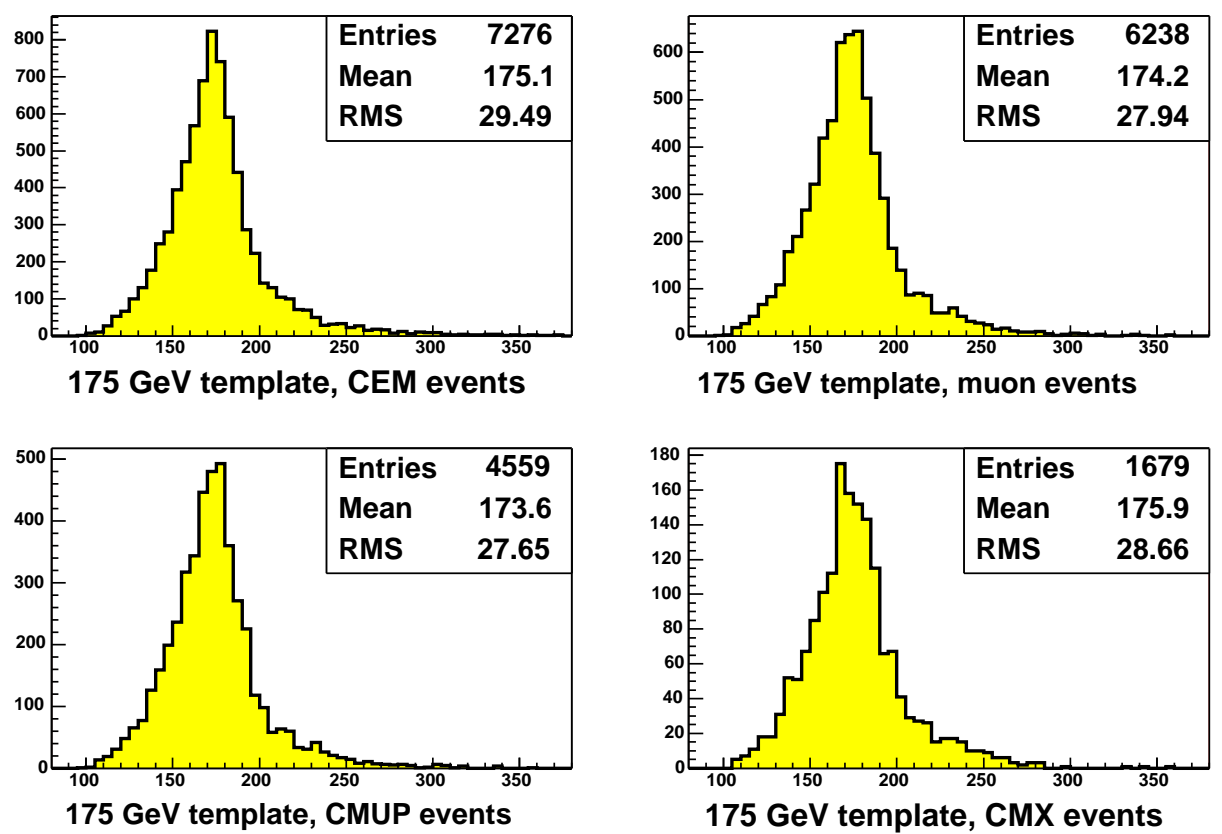

Figure 9.5: Reconstructed mass distributions of top events with all electrons (top left), all muons (top right), CMUP muons (bottom left), and CMX muons (bottom right).

\subsection{Kinematic Distributions}

We compare various kinematic distributions from the data events with the Monte Carlo predictions for combined signal and background events. The top mass analysis in the lepton+jets channel requires a good understanding of various objects such as leptons, $b$ jets, light jets, and neutrinos. Furthermore, kinematic distributions of reconstructed top and $t \bar{t}$ objects provide useful information about the properties of the top quark. This information could be used to check whether the top properties are consistent with Standard Model predictions.

Figure 9.6 shows the $p_{T}$ and $\eta$ distributions of the lepton (electron or muon) and Figure 9.7 shows the $E_{T}$ distribution of the leading jet and $E_{T}$ distribution. The data are compared with the Monte Carlo simulation with $175 \mathrm{GeV} / c^{2}$ top signal and background combined.

The $H_{T}$ distribution is shown in Figure 9.8. $H_{T}$ is defined as

$$
H_{T}=p_{T}^{e, \mu}+\notin_{T}+\Sigma_{i} E_{T}^{i-t h}{ }^{j e t},
$$

where jets include all the jets with $E_{T}>8 \mathrm{GeV}$ and $\left|\eta^{j e t}\right|<2$. In these distributions, jets 

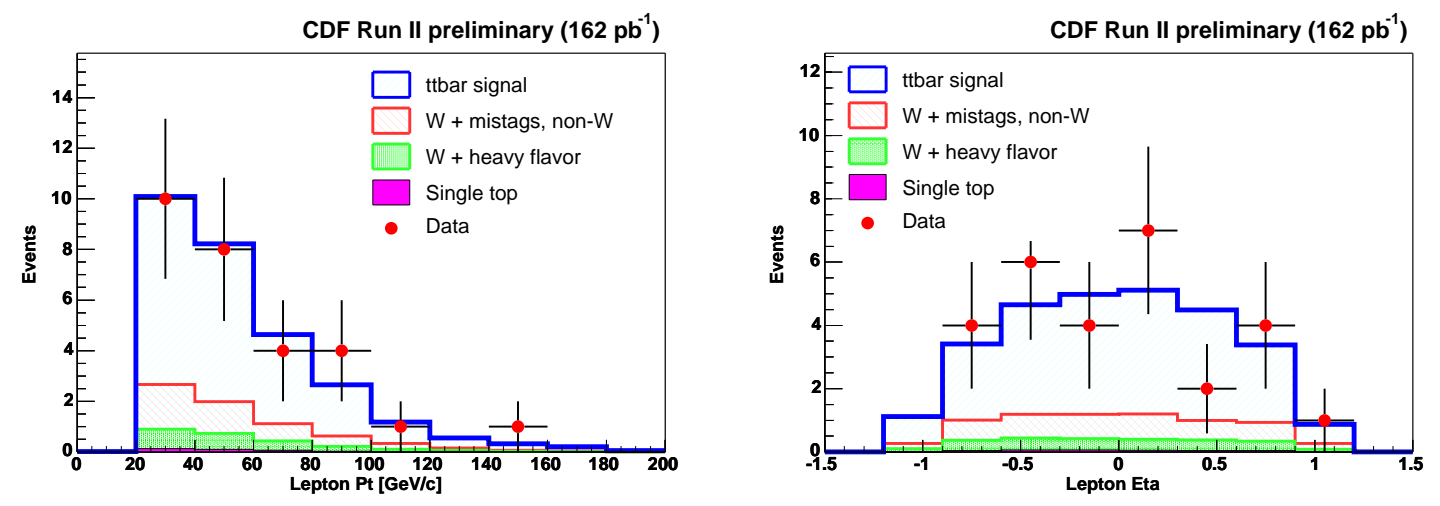

Figure 9.6: $p_{T}$ (left) and $\eta$ (right) distributions of the lepton $(e$ or $\mu)$ for $W+\geq 3.5$ jet events.
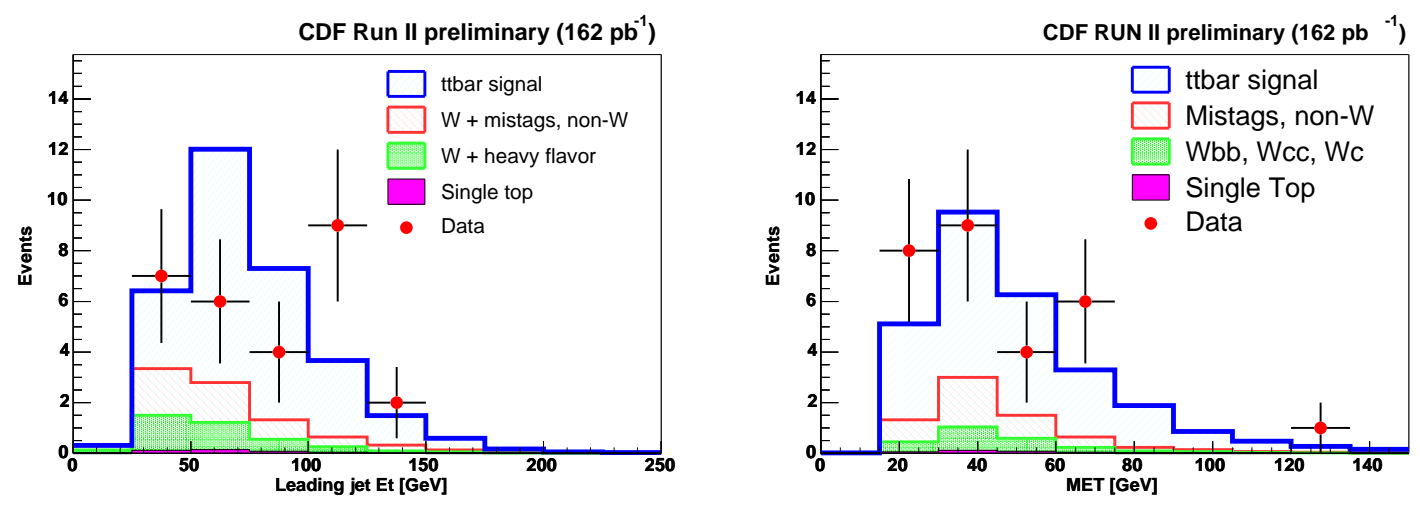

Figure 9.7: $E_{T}$ of the leading jets (left) and $\mathbb{E}_{T}$ (right) for $W+\geq 3.5$ jet events. 

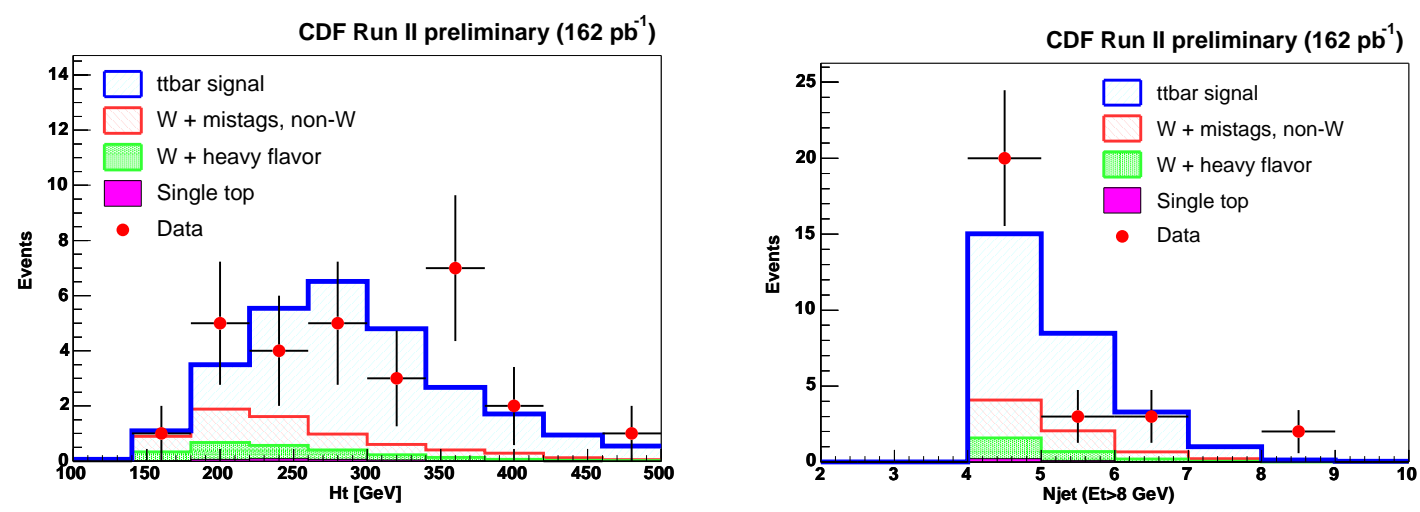

Figure 9.8: $H_{T}$ distribution (left) and number of tight + loose jets (right) for $W+\geq 3.5$ jet events.
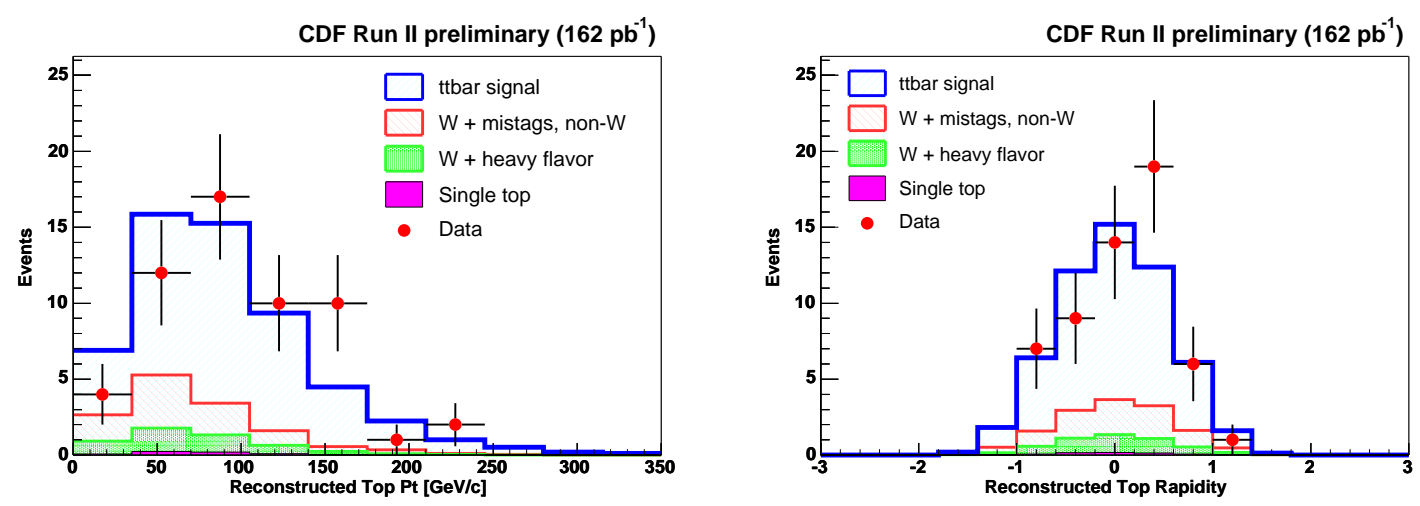

Figure 9.9: $p_{T}$ (left) and $\eta$ (right) distributions of the top for $W+\geq 3.5$ jet events.

and $\mathbb{E}_{T}$ are the quantities used for event selection (see chapter 4). Also shown in Figure 9.8 is the distribution of number of jets in the event $\left(E_{T}>8 \mathrm{GeV}\right)$.

Figure 9.9 shows the $p_{T}$ and $\eta$ distributions of reconstructed top quarks. The jet assignments are taken from the best $\chi^{2}$ combination in the top mass fitting. Thus the top-specific corrections as well as the $W$ mass constraint are applied to the jets.

The $p_{T}$ distribution of the reconstructed $t \bar{t}$ system is shown in Figure 9.10. As above, the jet assignments are taken from the best $\chi^{2}$ combination in the top mass fitting.

Finally, the mass of the reconstructed $t \bar{t}$ system is shown in Figure 9.11. All of the distributions show reasonable agreement between data and the Standard Model predictions. 

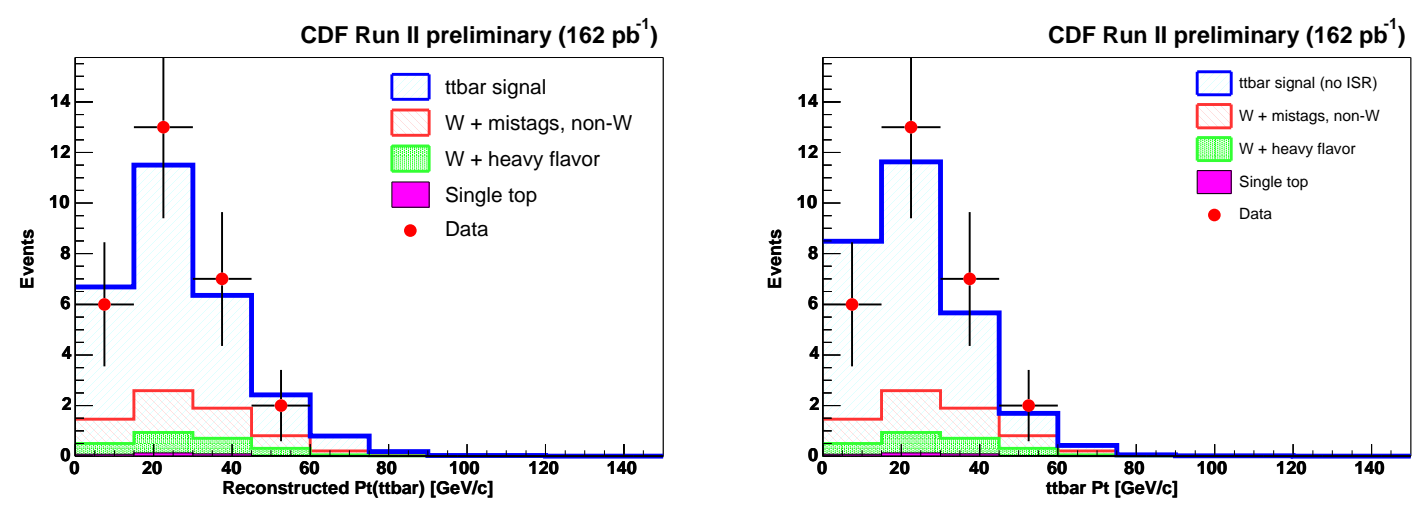

Figure 9.10: The $p_{T}$ distribution of the $t \bar{t}$ system using the default $175 \mathrm{GeV} / c^{2} t \bar{t}$ Herwig Monte Carlo sample (left plot). The right plot uses the $t \bar{t}$ Pythia Monte Carlo sample without ISR.

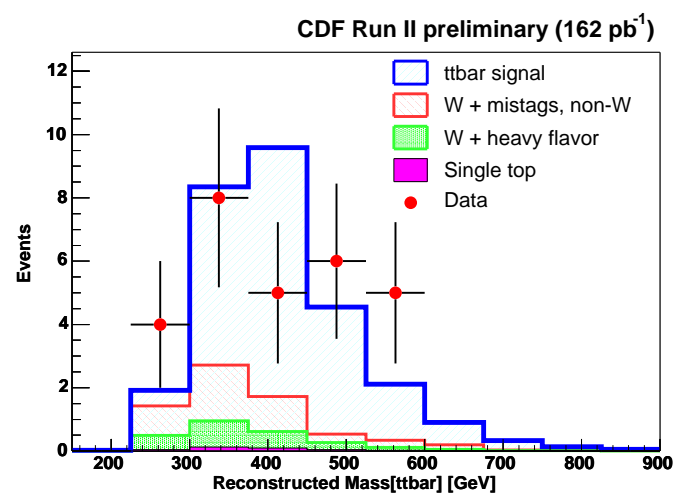

Figure 9.11: The mass of the $t \bar{t}$ system. 


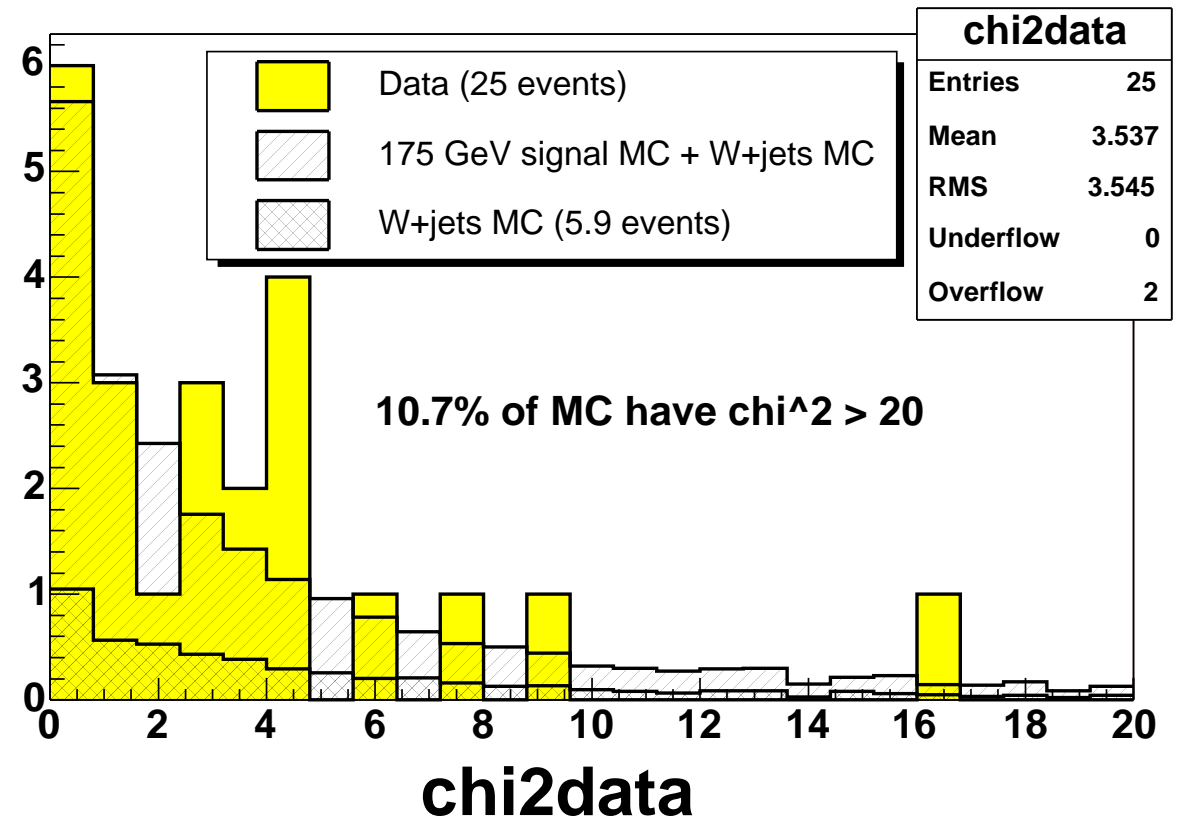

Figure 9.12: The $\chi_{\min }^{2}$ distributions of combined Monte Carlo sample of top and background (hatched histograms) and the data (yellow histogram).

\section{6 $\chi^{2}$ Check}

Figure 9.12 compares the $\chi_{\min }^{2}$ distribution in the mass reconstruction between the data and the signal + background samples. The data and simulated events are in good agreement, including the fraction of events with $\chi_{\min }^{2}>20$. 


\section{Chapter 10}

\section{Conclusions}

The top quark mass is measured using Run II data taken between March 2002 and September 2003. With the requirement of $W+\geq 3.5$ jets, we fit $28 t \bar{t}$ candidates to a mass of

$$
\mathrm{M}_{\text {top }}=174.9_{-7.7}^{+7.1} \text { (stat.) } \pm 6.5 \text { (syst.) } \mathrm{GeV} / c^{2} \text {. }
$$

There is a dominant systematic of $6.3 \mathrm{GeV} / c^{2}$, due to uncertainties in the jet energy scale. This systematic will become the largest source of uncertainty in the top mass measurement with only modest additional statistics.

We have not met our goal of matching the precision of the run I measurement with similar statistics. In part, we have been unlucky - the events did not fall in a statistically favorable distribution. Additionally, a large amount of work was done over many years to understand the CDF run I detector, identifying and reducing systematic uncertainties in many areas. That work is still underway for CDF II, especially in the area of jet energy systematics. A new version of the simulation code, with many improvements in the calorimeter description and jet simulation code, promises to reduce differences between data and Monte Carlo jets that contribute to the current large systematics. That and much more will be

needed to achieve $3 \mathrm{GeV} / c^{2}$ precision, combined over channels and experiments; but we have laid important groundwork, and that goal is within reach. 


\section{Appendix A}

\section{Pseudoexperiments}

We would often like to characterize the expected performance of the analysis in a statistically meaningful way, for example to get the expected statistical error, or to understand the shift in the top mass caused by a given systematic effect. We use large ensembles of sets of 28 Monte Carlo events which pass our cuts; each set of events is a pseudoexperiment. The full analysis machinery is used on each pseudoexperiment, and the top mass, with associated uncertainties, is extracted. So, for example, we use the median measured top mass for an ensemble of pseudoexperiments to understand the systematic effect on the top mass due to changing the jet energy scale by $4 \%$.

A problem arises. We would like to use thousands of pseudoexperiments to understand well the results of the analysis, but we would need $28 \times 1000^{\prime}$ s of events to make such an ensemble. A typical sample (see Figure 6.1) contains only $~ 7000$ events that pass all of our selection cuts. We use a form of random sampling with replacement to extend the power of our limited Monte Carlo samples [22]. By taking the same events in different combinations (different sets of 32) we can get thousands of pseudoexperiments that are somewhat statistically independent, though not as much so as thousands of completely disjoint sets of events. Since Monte Carlo generation, simulation, processing, and storage are prohibitively expensive, resampling allows us to maximize the power of reasonably sized MC datasets.

In practice, each event is fully characterized in this analysis by its reconstructed mass. Instead of keeping a vector of reconstructed masses and selecting a random entry from the vector 32 times, we keep a histogram of reconstructed masses and 32 times select a random mass according to the distribution in the histogram using the root function TH1: :GetRandom. This selects the bin of the histogram according to the cumulative 
distribution, and assumes a flat distribution of masses within each bin. ${ }^{1}$

Each pseudoexperiment consists of a number of masses from signal events, and a number of masses from background events, whose sum is a fixed $N$. (For the 3.5-jet sample, $N=28$.) The number of background events is chosen at random using the same constraint that will be applied in the likelihood fit, a Gaussian fluctuation followed by a Poisson fluctuation. The background masses are taken as described above from a background template, and the signal masses are taken as described above from a signal template. When a systematic is being estimated, both the signal and background templates should be varied in the same way.

When we use an ensemble of pseudoexperiments to measure the shift in top mass due to a particular systematic source, we would like to understand the uncertainty on our determination. $\left(1.0 \pm 1.0 \mathrm{GeV} / c^{2}\right.$ is a very different result from $1.0 \pm 0.1 \mathrm{GeV} / c^{2}$ when no systematic shift is expected.) We define the shift in top mass as the difference between the median measured top mass for pseudoexperiments drawn from the systematically shifted reconstructed mass histogram, and the median measured top mass for pseudoexperiments drawn from the default reconstructed mass histogram. There are two general cases: when the systematically shifted reconstructed mass histogram comes from the same MC sample as the base reconstructed mass histogram, and when they come from different MC samples. An example of the first case is jet systematics, where the systematically shifted masses use the same events as the default masses, but with shifted jet corrections. An example of the second case is ISR systematics, where there is no choice but to generate and simulate an entirely new sample with different ISR parameters in the generator. In both cases, there is a simple statistical uncertainty on the median of each ensemble of measured top masses from pseudoexperiments. But this uncertainty can be made arbitrarily small by performing arbitrarily many pseudoexperiments. We clearly have another source of uncertainty due to the limited statistics of the underlying sample, from which the reconstructed mass histograms were derived. In the first case described above, that uncertainty is highly correlated between the sytematically shifted and the default samples, so the uncertainty on the difference between the two medians is taken to be negligible. On the other hand, when the two reconstructed mass distributions are derived from completely independent sets of

\footnotetext{
${ }^{1}$ This may be a source of residual bias in the pseudoexperiment results. The bins in our reconstructed mass histograms have $5 \mathrm{GeV} / c^{2}$ width by default, and the assumption of a flat distribution within such a large bin can have a significant effect, especially in bins where the true distribution is changing rapidly.
} 
events, that uncertainty is uncorrelated between the two and must be accounted for. As an estimate of the uncertainty on the median of an ensemble of pseudoexperiments due to the limited statistics of the sample of reconstructed masses from which they were derived, we use the uncertainty on the mean of the reconstructed mass histogram, namely $R M S / \sqrt{N}$, where $N$ is the number of entries (events) in the histogram. 


\section{Appendix B}

\section{Pull Distributions and 68\% coverage}

It has been a concern that the pull widths from pseudoexperiments using Monte Carlo samples tended to be larger than one. This led to various studies in which we confirmed that the fundamental result of our fit, the likelihood curve, is valid. However, that likelihood curve is in general non-Gaussian, and large ensembles of pseudoexperiments yield pull widths greater than one. Similarly, the $\pm 1 \sigma$ uncertainties we were using did not provide $68 \%$ coverage of the true mass. But many physicists expect this property of Gaussian errors to obtain even in the case of non-Gaussian errors. Therefore we have scaled our reported statistical uncertainty to provide $68 \%$ coverage as described below.

A pull distribution with unit width can be obtained whenever the measured parameter has well estimated Gaussian errors. But the likelihood curve obtained using our method is in general non-Gaussian (i.e. the $-\ln L$ is in general non-parabolic). The nonGaussian likelihood can be due to several factors.

Background The presence of background can lead to likelihoods that are shallow on the low-mass side, since events at low mass (generated as background events) could be due instead to low-mass signal.

Low statistics The limited statistics of each pseudoexperiment lead to various complicated reconstructed mass shapes, such as double peaks, plateaus, holes in the distribution, etc. The likelihood curve for each pseudoexperiment will reflect to some degree the mass distribution. 
Templates The non-Gaussian shape of the signal templates (and their parameterization) means that the likelihood curve for even a single event will be non-Gaussian.

But when a likelihood curve is non-Gaussian, it cannot be described with a single number (the Gaussian uncertainty). We had been using errors defined by the masses $M_{+}$ and $M_{-}$at which the $\log$-likelihood has changed by $1 / 2$ unit from its minimum, which provide one definition of the statistical uncertainty for a general likelihood curve. However, this definition does not necessarily provide pull distributions with width 1 , or $68 \%$ coverage of the correct value. In particular, if the $-\ln L$ tends to be shallower than a parabola ( $L$ is wider than a Gaussian), the pulls will be too wide since events several "sigma" away from the minimum are more likely than they would be in a Gaussian distribution. Even in the absence of background, and with a Gaussian parent distribution (template), typically wider-than-Gaussian likelihoods have been reproduced in a toy Monte Carlo model when the number of events per experiment is small [23].

We verified that the tails and non-gaussian shape of the pull distributions disappear when considering large statistics. Pull distributions for pseudoexperiments with 210 signal events $\left(\mathrm{M}_{\mathrm{top}}=175 \mathrm{GeV} / \mathrm{c}^{2}\right)$ and no background are shown in Figure B.1, upper left. The pull widths are consistent with one. Figure B.1, upper right shows the same with 70 background events added per experiments. The pull widths remain consistent with one. Thus the non-Gaussian shape of a single-event likelihood becomes increasingly gaussian as the statistics increases, as predicted by the central limit theorem, and we conclude the current shape of the pull width is due to low statistics. Note that a bias appears in the pull mean with higher statistics (i.e., smaller errors). There is no apparent bias with the current event sample size (as shown in Figure 6.8), but from these plots we can expect the bias to become more noticeable as the sample size increases. We have noticed that the bias disappears when selecting the pseudo-data from the template fit functions instead of the template histograms (see Figure B.1, bottom). This suggests there is a remaining discrepancy between the template shape and their fit functions to which we are not sensitive yet, and we will need to investigate this effect more thoroughly in the future.

Having satisfied ourselves that the behavior we see in pseudoexperiments is reasonable, we scaled the errors obtained using $\Delta \ln \mathcal{L}=1 / 2$ so that $68 \%$ coverage of the true value is expected. In particular, we investigate pseudoexperiments for which the generated 


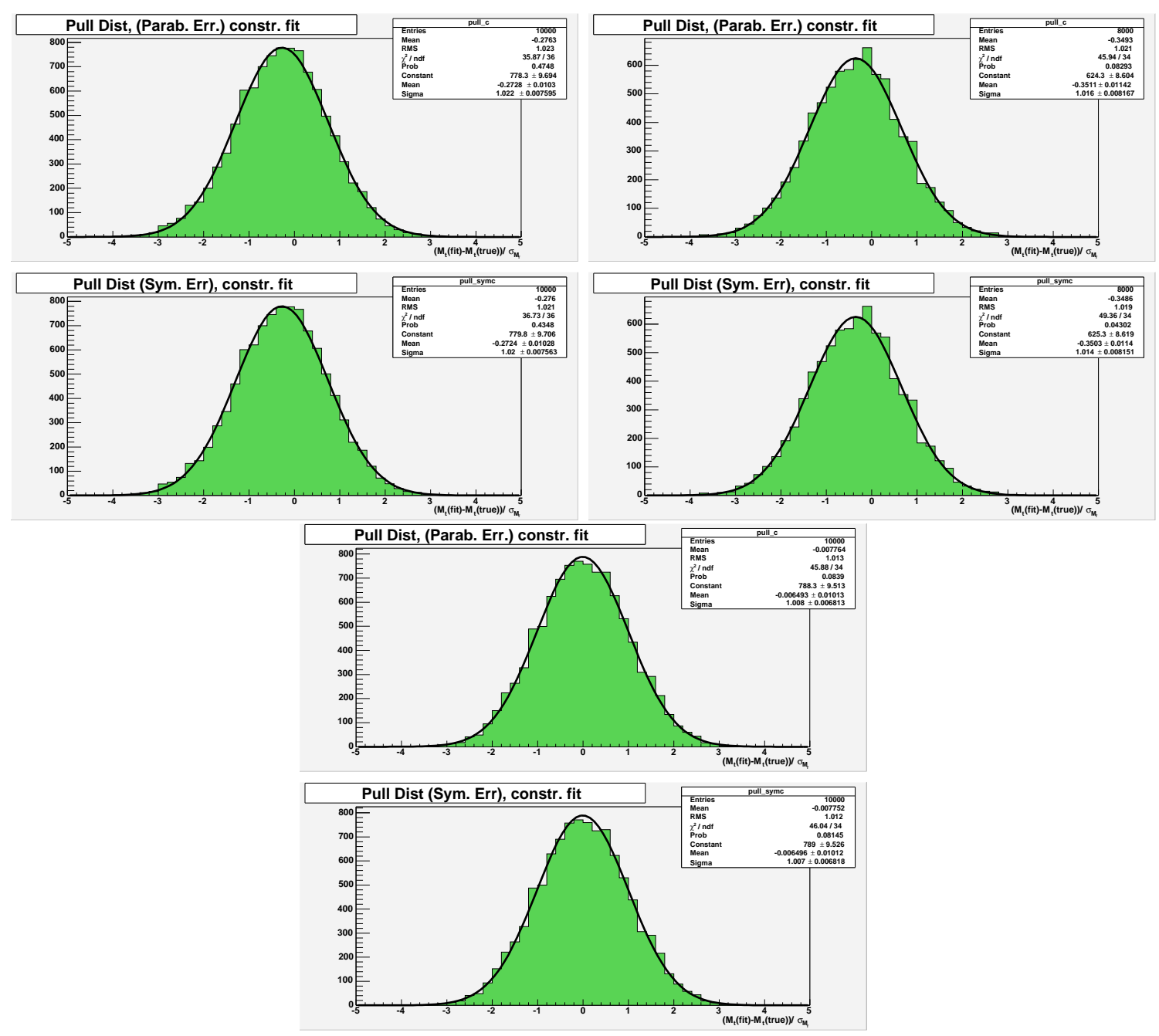

Figure B.1: Top left: Pull distributions for parabolic errors (upper plot) and symmetrized errors (lower plot) of pseudoexperiments each containing 10 times the signal events statistics (210 events) and no background events. Top right: Pull distributions for pseudoexperiments containing 210 events including 70 background events. Bottom: Pull distributions for pseudoexperiments containing 210 events with no background, where pseudodata events were drawn from the fitted signal p.d.f., rather than from the $175 \mathrm{GeV} / c^{2}$ template histogram. 

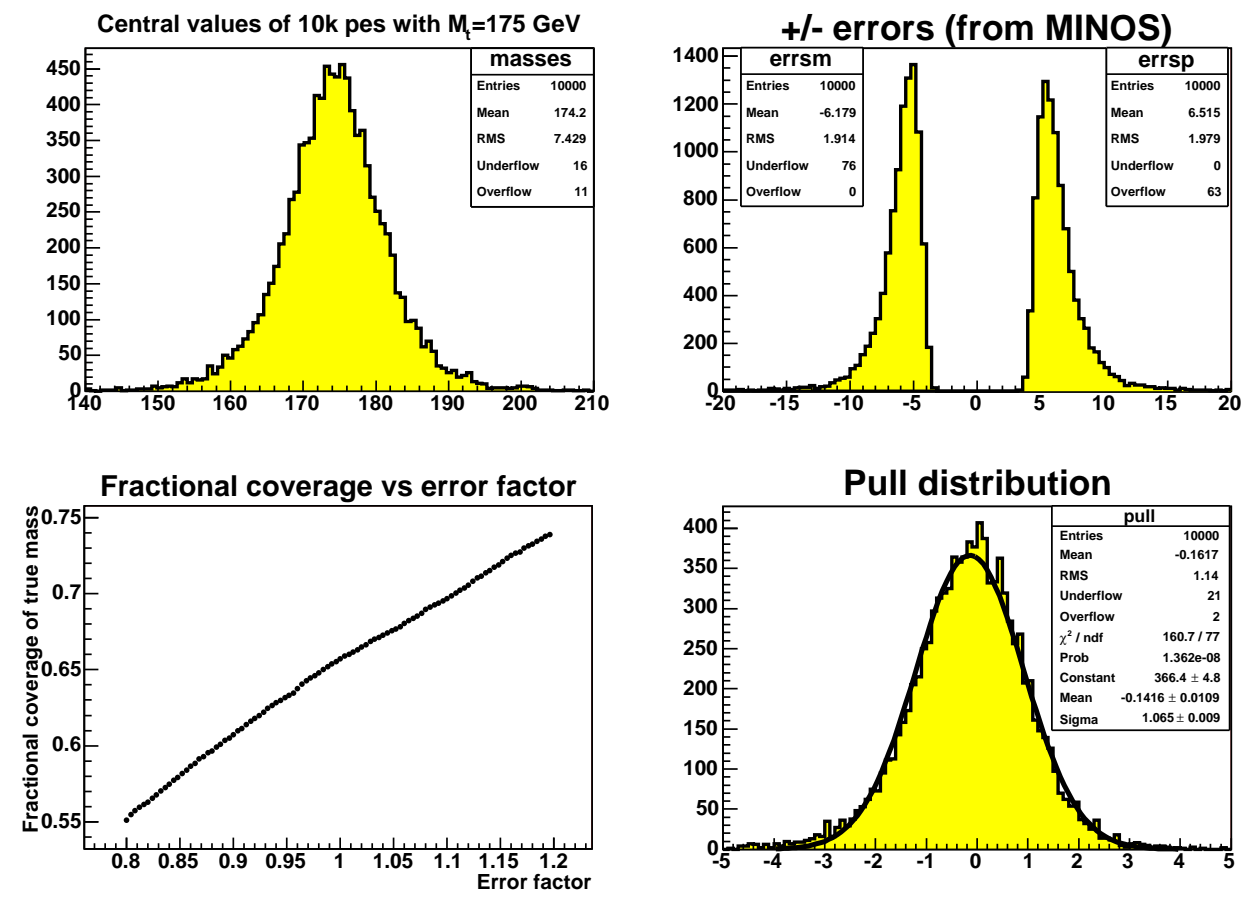

Figure B.2: Ten thousand pseudoexperiments were performed with a generated mass of $175 \mathrm{GeV} / c^{2}$. The top left plot shows the central values of the likelihood fits. At top right the positive and negative errors determined using $\Delta \ln \mathcal{L}$ are plotted. In the bottom right frame is the pull distribution using these raw uncertainties. The bottom left shows the fraction of the pseudoexperiments for which the true mass lies within the one sigma errors, as a function of the multiplicative factor applied to the raw errors.

mass is $175 \mathrm{GeV} / c^{2}$, very close to the measured central value of $174.9 \mathrm{GeV} / c^{2}{ }^{1}$ Some information from the 10,000 such pseudoexperiments is shown in Figure B.2. In particular, the bottom left curve shows the fraction of pseudoexperiments for which the true mass (175 GeV/c $c^{2}$ ) lies within the $\pm 1 \sigma$ errors, as a function of a multiplicative factor applied to the raw uncertainties. A fractional coverage of $68.3 \%$ is found when the errors are scaled by 1.065. Therefore the quoted statistical uncertainty on our primary measurement is obtained by scaling the $\Delta \ln \mathcal{L}$ errors by 1.065 .

\footnotetext{
${ }^{1}$ Unfortunately, there is no obvious (to us) prescription for achieving $68 \%$ coverage from the quoted errors. There doesn't seem to be a way to extract such errors directly from a given likelihood curve, so some ensemble of pseudoexperiments must be used. But which ensemble and how to generate or scale the errors is somewhat ambiguous.
} 


\section{Bibliography}

[1] T. Affolder et al. Measurement of the top quark mass with the Collider Detector at Fermilab. Phys. Rev., D63:032003, 2001. hep-ex/0006028.

[2] Dhiman Chakraborty, Jacobo Konigsberg, and David L. Rainwater. Review of top quark physics. hep-ph/0303092, 2003.

[3] S. Willenbrock. Studying the top quark. Rev. Mod. Phys., 72:1141-1148, 2000. hepph/0008189.

[4] M. Beneke et al. Top quark physics. TH 2000-100, CERN, March 2000. hep$\mathrm{ph} / 0003033$.

[5] F. Abe et al. Observation of top quark production in anti-p p collisions. Phys. Rev. Lett., 74:2626-2631, 1995. hep-ex/9503002.

[6] S. Abachi et al. Observation of the top quark. Phys. Rev. Lett., 74:2632-2637, 1995. hep-ex/9503003.

[7] Jens Erler and Paul Langacker. Electroweak model and constraints on new physics. Phys. Lett., B592:1, 2004. hep-ph/0407097.

[8] Elizabeth H. Simmons. Thinking about top: Looking outside the standard model. hep-ph/9908511, 1998.

[9] T. Affolder et al. CDF central outer tracker. PUB 03-355-E, Fermilab, November 2003.

[10] A. Sill. CDF Run II silicon tracking projects. Nucl. Instrum. Meth., A447:1-8, 2000.

[11] L. Balka et al. The CDF central electromagnetic calorimeter. Nucl. Instrum. Meth., A267:272, 1988 . 
[12] S. Bertolucci et al. The CDF central and endwall hadron calorimeter. Nucl. Instrum. Meth., A267:301, 1988.

[13] M. G. Albrow et al. The CDF plug upgrade electromagnetic calorimeter: Test beam results. Nucl. Instrum. Meth., A480:524-546, 2002.

[14] G. Ascoli et al. CDF central muon detector. Nucl. Instrum. Meth., A268:33, 1988.

[15] F. Abe et al. The topology of three jet events in anti-p p collisions at $\mathrm{s}^{* *}(1 / 2)=$ 1.8-tev. Phys. Rev., D45:1448-1458, 1992.

[16] G. Corcella, I. G. Knowles, G. Marchesini, S. Moretti, K. Odagiri, P. Richardson, M. H. Seymour, and B. R. Webber. Herwig 6.5. JHEP, 01:010, 2001. [hep-ph/0011363]; hep$\mathrm{ph} / 0210213$.

[17] Torbjorn Sjostrand, Leif Lonnblad, and Stephen Mrenna. Pythia 6.2: Physics and manual. TP 01-21, LU, 2001. hep-ph/0108264.

[18] Michelangelo L. Mangano, Mauro Moretti, Fulvio Piccinini, Roberto Pittau, and Antonio D. Polosa. ALPGEN, a generator for hard multiparton processes in hadronic collisions. JHEP, 07:001, 2003.

[19] T. Affolder et al. Measurement of the $\mathrm{t}$ anti-t production cross section in $\mathrm{p}$ anti-p collisions at $\mathrm{s}^{* *}(1 / 2)=1.8$-tev. Phys. Rev., D64:032002, 2001. hep-ex/0101036.

[20] F. James. MINUIT function minimization and error analysis, reference manual, version 94.1. Long Writeup D506, CERN, August 1998.

[21] T. Affolder et al. Measurement of the top quark $\mathrm{p}(\mathrm{t})$ distribution. Phys. Rev. Lett., $87: 102001,2001$.

[22] Roger Barlow. SLUO lectures on statistics and numerical methods in HEP, Lecture 6: Resampling and the bootstrap. http://www.hep.man.ac.uk/u/roger/sluo6.ps, August 2000 .

[23] John Conway. Private Communication, April 2004. 